\title{
DESIGN OF HIGH EFFICIENCY BLOWERS FOR FUTURE
}

\section{AEROSOL APPLICATIONS}

\author{
A Thesis \\ by \\ RAMAN CHADHA \\ Submitted to the Office of Graduate Studies of \\ Texas A\&M University \\ in partial fulfillment of the requirements for the degree of \\ MASTER OF SCIENCE
}

December 2005

Major Subject: Mechanical Engineering 


\title{
DESIGN OF HIGH EFFICIENCY BLOWERS FOR FUTURE
}

\section{AERSOSOL APPLICATIONS}

\author{
A Thesis \\ by \\ RAMAN CHADHA \\ Submitted to the Office of Graduate Studies of \\ Texas A\&M University \\ in partial fulfillment of the requirements for the degree of \\ MASTER OF SCIENCE
}

\begin{abstract}
Approved by:
Chair of Committee, Gerald L. Morrison

Committee Members, Andrew R. McFarland

Yassin A. Hassan

John Haglund

Head of Department, Dennis O’Neal
\end{abstract}

December 2005

Major Subject: Mechanical Engineering 


\begin{abstract}
Design of High Efficiency Blowers for

Future Aerosol Applications. (December 2005)

Raman Chadha, B-En., IIT-Kanpur, India

Chair of Advisory Committee: Dr. Gerald Morrison
\end{abstract}

High efficiency air blowers to meet future portable aerosol sampling applications were designed, fabricated, and evaluated. A Centrifugal blower was designed to achieve a flow rate of $100 \mathrm{~L} / \mathrm{min}\left(1.67 \times 10^{-3} \mathrm{~m}^{3} / \mathrm{s}\right)$ and a pressure rise of $4 " \mathrm{WC}(1000 \mathrm{~Pa})$. Commercial computational fluid dynamics (CFD) software, FLUENT 6.1.22, was used extensively throughout the entire design cycle. The machine, Reynolds number $(\mathrm{Re})$, was around $10^{5}$ suggesting a turbulent flow field. Renormalization Group (RNG) $\kappa-\varepsilon$ turbulent model was used for FLUENT simulations. An existing design was scaled down to meet the design needs. Characteristic curves showing static pressure rise as a function of flow rate through the impeller were generated using FLUENT and these were validated through experiments.

Experimentally measured efficiency $\left(\eta_{E X P}\right)$ for the base-design was around $10 \%$. This was attributed to the low efficiency of the D.C. motor used. CFD simulations, using the $\kappa-\varepsilon$ turbulent model and standard wall function approach, over-predicted the pressure rise values and the percentage error was large.

Enhanced wall function under-predicted the pressure rise but gave better agreement (less than $6 \%$ error) with experimental results. CFD predicted a blower scaled $70 \%$ in planar direction $(X Z)$ and $28 \%$ in axial direction $(Y)$ and running at $19200 \mathrm{rpm}$ (70xz_28y@19.2k) as the most appropriate choice. The pressure rise is $1021 \mathrm{~Pa}$ at the 
design flow rate of $100 \mathrm{~L} / \mathrm{min}$. FLUENT predicts an efficiency value based on static head $\left(\eta_{F L U}\right)$ as $53.3 \%$. Efficiency value based on measured static pressure rise value and the electrical energy input to the motor $\left(\eta_{E X P}\right)$ is $27.4 \%$. This is almost a $2 X$ improvement over the value that one gets with the hand held vacuum system blower. 


\section{DEDICATION}

This work is the outcome of the unconditional love and support that my parents, Mrs. Suchita Chadha and Mr. Vijay Kumar Chadha, have for me. Their blessings, encouragement, and the ability to see the good in me and believe in me - even when I had doubts about myself, have brought me to the present stage.

Finally, every deed of mine reminds me of a higher guiding power above: He who is instrumental for all the change. It is at all times that I seek his blessings! 


\section{ACKNOWLEDGEMENTS}

I would like to express my gratitude to my advisor, Dr. Gerald L. Morrison, for the long fruitful discussions we had in his office. His cheerful 'Howdy!' when you knocked on his door always set the tone for the meetings. His patience, encouragement, and his ability to mold you and point in the right direction, are the only reasons this work could be completed. Everything I know about turbomachines, I have learned from him.

Thanks are due to Dr. Andrew R. McFarland, who had the vision for this project even before I started working on it. His cheerful smile always uplifts you and raises your performance level. His belief in me was my motivation all along. Thank you!

I would like to thank the members of my committee for their time and guidance, especially Dr. John Haglund. Managing the whole ATL Lab but still having those 'couple of minutes' whenever you needed his time was appreciated. He set the pace during the biweekly meetings and provided fruitful insights all along.

Finally, thanks are due to all of the members of the ATL, for accepting me as one of them: Ben Thein for helping me with the lab geography and whereabouts, John Vaughan and Michael Salomon for their patience during the drafting and for generating beautiful drawings, and Shishan, Vishnu, and Sridhar for the discussions we had on CFD. Their knowledge about CFD and their patience in helping me understand CFD is commendable. Thanks to Ashley, Clint, Manpreet, Ref, Rohit, Satya, Suvi, Travis, and Youngjin for the good times we had together. Thank you all! 


\section{TABLE OF CONTENTS}

Page

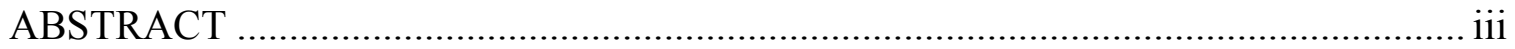

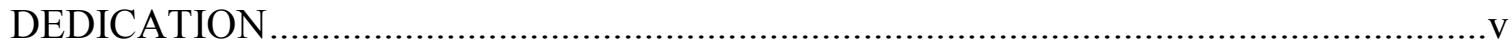

ACKNOWLEDGEMENTS .............................................................................. vi

TABLE OF CONTENTS ................................................................................. vii

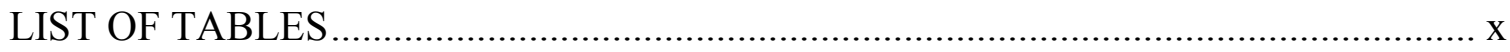

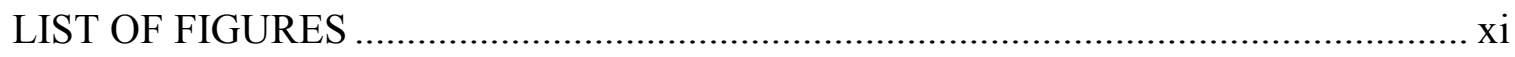

NOMENCLATURE, ABBREVIATIONS, AND UNITS........................................ xiv

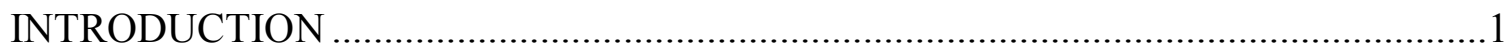

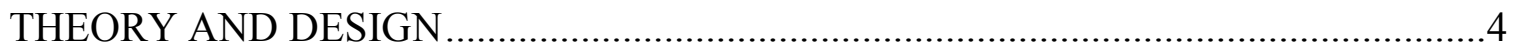

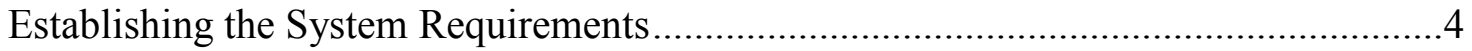

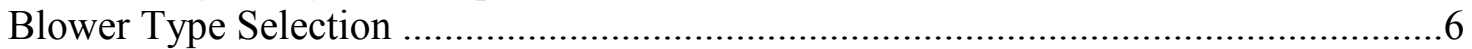

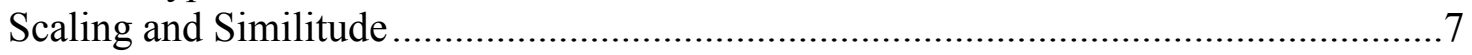

Specific Speed and Optimum Geometry .................................................................. 11

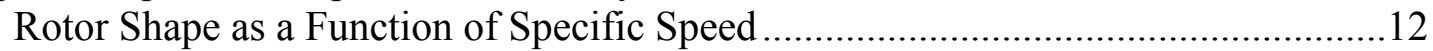

Selection of Optimum Specific Speed Values .................................................... 14

Estimating Head Rise $\left(\Delta H_{t o t}\right)$ across an Impeller ................................................. 15

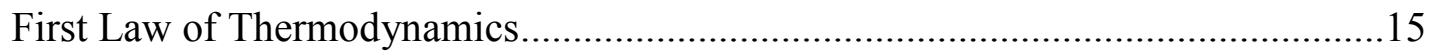

Sample Calculations for Impeller Specific Speed ................................................ 17

Single Stage Impeller Calculations .......................................................................... 18

Two Stage Impeller Calculations.........................................................................19

Size Calculation for Single Stage Centrifugal Blower …...................................20

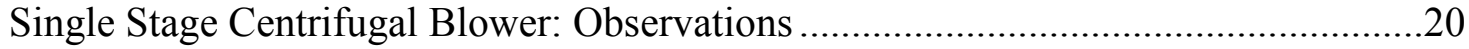

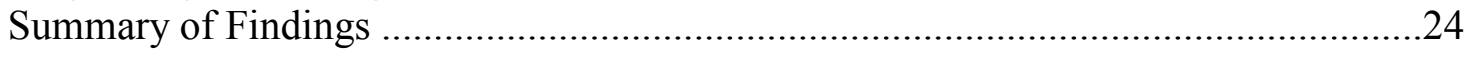

BLOWER BASE-DESIGN SELECTION ...............................................................25

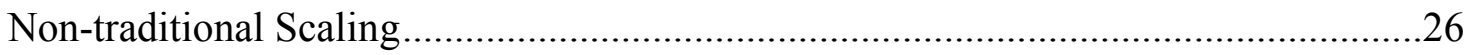

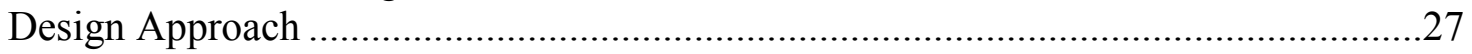

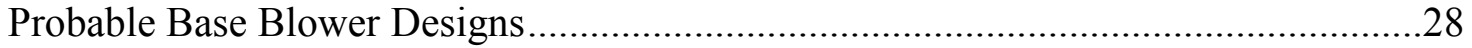

Selection of the Hand-held Vacuum System (HVS) Blower: Justification ....................30

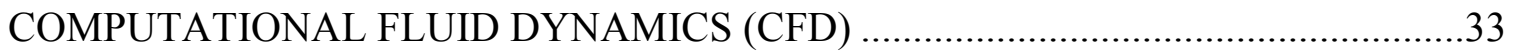




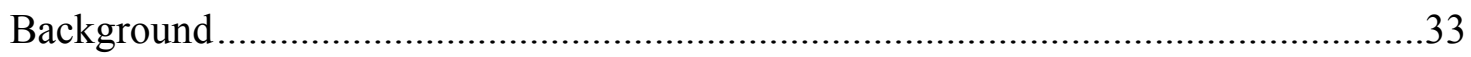

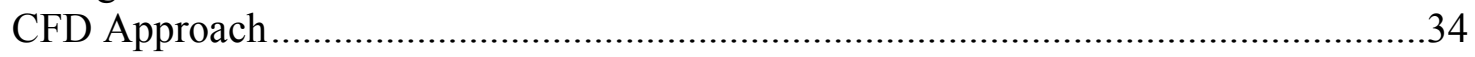

FLUENT 6.1.22 - Introduction .......................................................................... 35

Blower Geometry for CFD Simulations ................................................................ 36

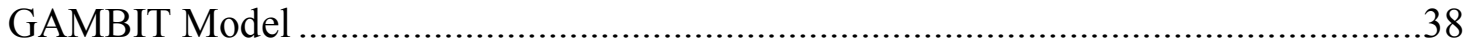

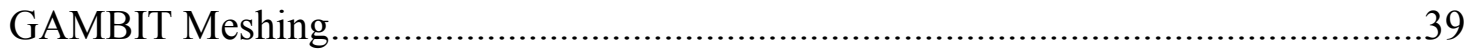

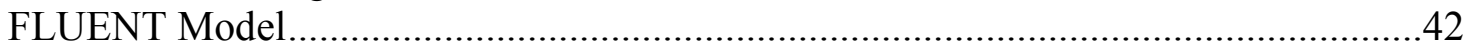

Turbulence Model ........................................................................................4

Inlet and Outlet Conditions..............................................................................43

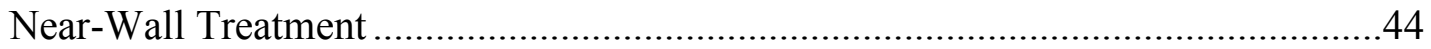

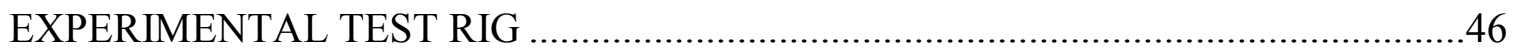

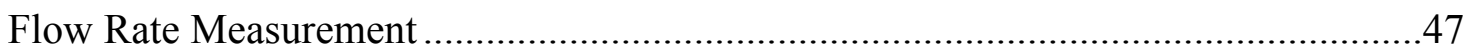

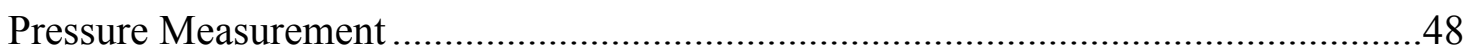

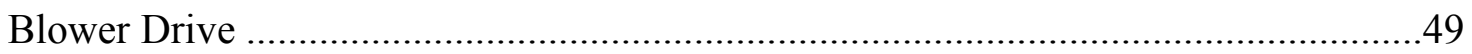

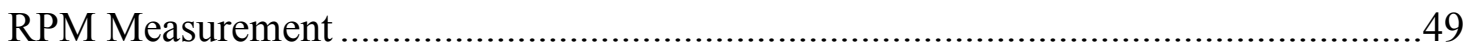

BASE-DESIGN SIMULATION AND EXPERIMENTAL DATA COMPARISON.......50

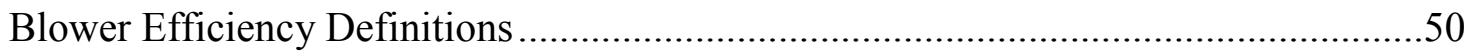

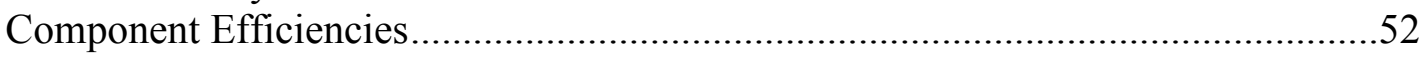

FLUENT and Experimental Efficiency Definition.....................................................55

Hand-held Vacuum System Blower (100xz_100y) .................................................56

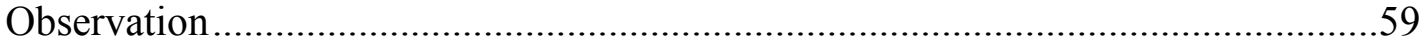

FLUENT SIMULATIONS FOR DIFFERENT SCALING CASES …………………......60

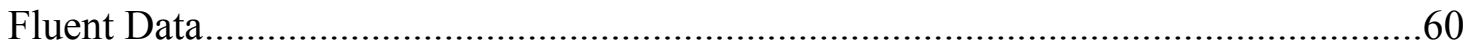

Non-dimensional Study for Blower Simulation Results ..............................................63

EXPERIMENTAL VALIDATION FOR 65XZ_26Y@20K BLOWER ...........................67

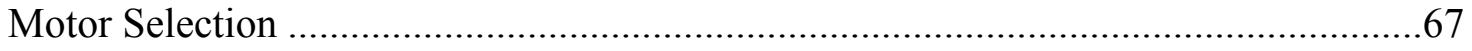

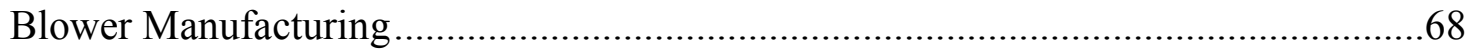

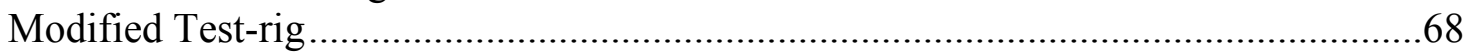

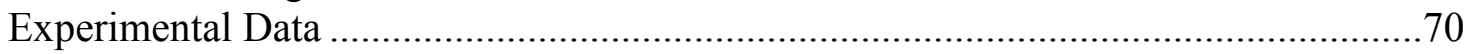

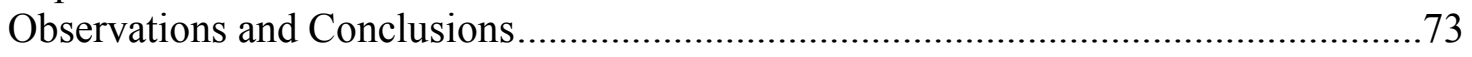

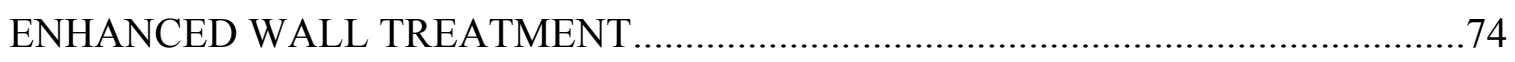

Enhanced Wall Treatment FLUENT Simulations for 65xz_26y@20k Blower ............74

Conservative Efficiency Estimates for 65xz_26y@20k Blower ...................................76

Resizing of Blower Using Enhanced-wall Treatment ..................................................78 
EXPERIMENTAL VALIDATION FOR 70XZ_28Y@20K BLOWER .80

Results. .80

Conservative Efficiency Estimates for 70xz_28y@20k Blower .82

Observations and Conclusions

FINAL BLOWER PROTOTYPE: DESIGN AND TESTING .85

Prototype Design Setup: Blower 70xz_28y with $\delta_{\text {inlet }}=0.009 "$ and $\delta_{\text {shroud }}=0.012^{\prime \prime} \ldots .85$

Results .86

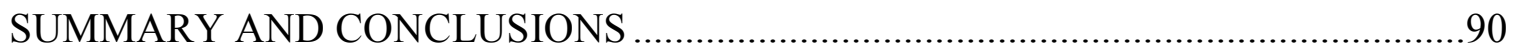

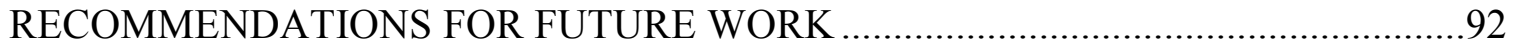

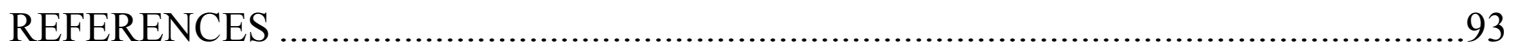

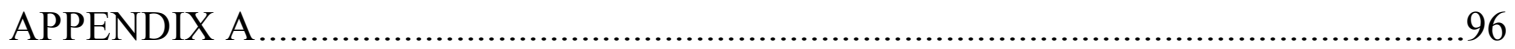

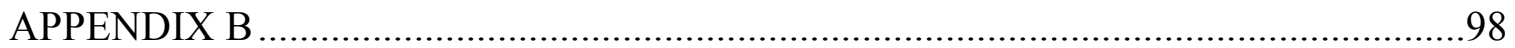

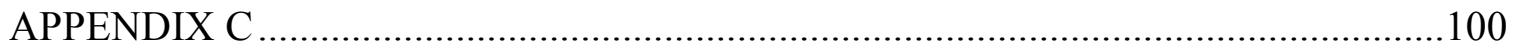

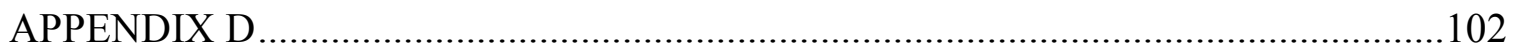

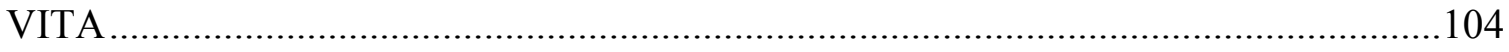




\section{LIST OF TABLES}

Page

Table 1 Specific speed values and blower sizes at different rpms.............................. 22

Table 2 Ratio of pressure head and theoretical velocity head for different rpm and impeller diameter combinations................................................................. 23

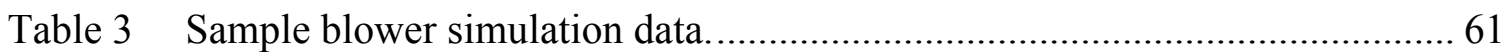

Table 4 Efficiency values for different scaled blower at design flow rate. ................. 62

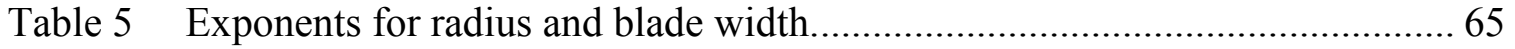

Table 6 Blower efficiency estimates at design flow rate. ......................................... 76

Table 7 Static pressure values for different sized blowers at design flow rate............ 78

Table 8 Blower efficiency estimates at design flow rate ....................................... 83

Table 9 Efficiency estimates for final prototype blower at design flow rate...............89

Table A-1 Values chosen for mass flow calculation. ................................................... 96

Table D-1 Sample data sheet for final prototype blower at design rpm. ...................... 103 


\section{LIST OF FIGURES}

Page

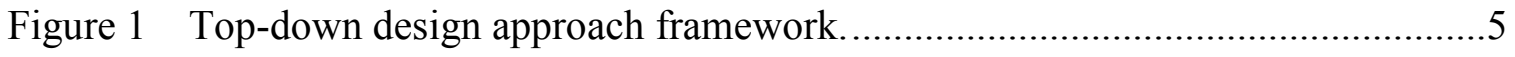

Figure 2 Cross-sections of different pumps: (a) Radial flow (b) Mixed flow (c) Axial flow (Hydraulics Institute).

Figure 3 Defining the geometry of a pump stage (Karassik et al. 2000, reprinted with permission of McGraw-Hill).

Figure 4 Optimum geometry as a function of BEP specific speed (Karassik et al. 2000, reprinted with permission of McGraw-Hill).

Figure 5 Specific speed values for different pump designs (Hydraulics Institute).........13

Figure 6 Efficiency values for pump with different specific speeds (Karassik et al. 2000, reprinted with permission of McGraw-Hill).

Figure 7 Energy balance across a control volume for a rotating impeller. 16

Figure 8 Efficiency $\left(\eta_{\text {overal }}\right)$ of centrifugal pumps versus specific speed, size, and shape (Anderson 1980). 23

Figure 9 Turbo blowers with backward curved blades (shroud removed).

Figure 10 Hand-held vacuum system centrifugal blower (a) Top-view (b) Side-view showing the blades.

Figure 11 Variety of common pump impeller meridional views from low to high specific speeds $(\mathrm{a}-\mathrm{f})$ and with inducer $(\mathrm{g})$....

Figure 12 Impeller with strong inlet curvature (a) Separation with reattachment (b) Complete separation without reattachment.

Figure 13 Common centrifugal fan blade shapes.

Figure 14 Base blower dimensions $(\mathrm{mm})$.

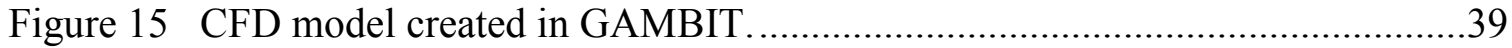

Figure 16 CFD model mesh: (a) Top-view (b) Side-view............................................40

Figure 17 Mesh quality: Skewness value for mesh cells..........................................42

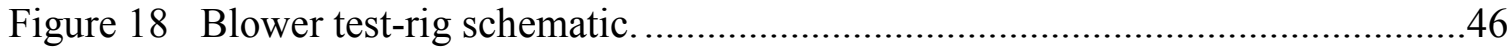

Figure 19 Blower performance measurement test-rig. .............................................47 
Figure 20 Pressure recovery for different venturi types (FLOW-DYNE

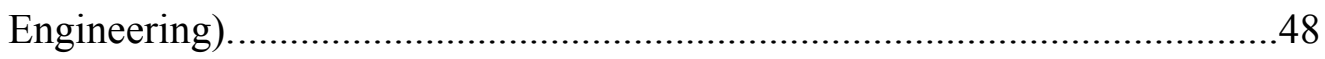

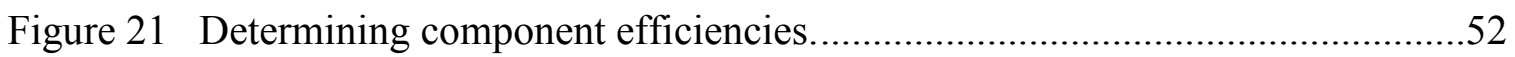

Figure 22 FLUENT performance curves for 100xz_100y blower................................56

Figure 23 Experimental performance curves for $100 \mathrm{xz} \_100 \mathrm{y}$ blower. .........................57

Figure 24 Experimental electrical power consumption curves for 100xz_100y

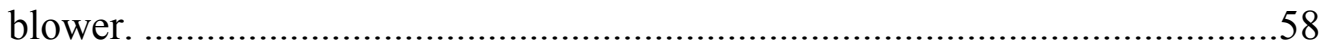

Figure 25 FLUENT -vs. - Experimental data for 100xz_100y blower. .........................58

Figure 26 Experimental efficiency $\left(\eta_{E X P}\right)$ values for $100 x_{-} 100 y$ blower. ...................59

Figure 27 FLUENT performance curve for different-scaled blowers. ...........................60

Figure 28 FLUENT efficiency values $\left(\eta_{F L U}\right)$ for different scaled blowers. ..................61

Figure 29 FLUENT performance curves for 65xz_26y@20k blower..........................63

Figure 30 FLUENT non-dimensional modified head coefficient curve........................65

Figure 31 Test rig setup with Al-1: SC front plate (dimensions in $\mathrm{mm}$ ).....................69

Figure 32 Experimental performance curves for 65xz_26y blower with Al-2: BC

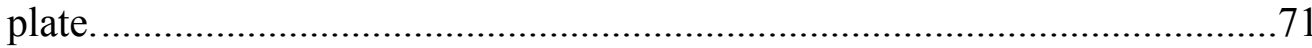

Figure 33 Experimental performance curves for $65 \mathrm{xz} \_26 \mathrm{y}$ blower with Al-1: SC plate.

Figure 34 Experimental electrical power consumption curves for 65xz_26y blower with different front plates

Figure 35 Comparison between performance curves for FLUENT, Al-1: SC, and Al2: BC front plates.

Figure 36 Performance comparison for $65 \mathrm{xz}_{2} 26 \mathrm{y}$ blower: Enhanced wall treatment vs. - experimental results with A1-1: SC front plate.

Figure 37 FLUENT static pressure rise value for $65 \mathrm{xz}_{2} 26 \mathrm{y}$ blower for different wall treatments and comparison with Al-1: SC experimental data. . .75

Figure 38 Efficiency values for pump with different specific speeds (Karassik et al. 2000, reprinted with permission of McGraw-Hill). 
Figure 39 Experimental performance curve for 70xz_28y blower with Al-2: BC plate.

Figure 40 Experimental electrical power consumption curves for 70xz_28y blower with Al-2: BC front plate.

Figure 41 Experimental efficiency $\left(\eta_{E X P}\right)$ curves for $70 x z \_28 y$ blower with Al-2: $\mathrm{BC}$ front plate. .82

Figure 42 Final blower prototype test setup.

Figure 43 Experimental performance curves for final-prototype blower size 70xz_28y.

Figure 44 Experimental electrical power consumption curves for final-prototype.........87

Figure 45 Experimental efficiency $\left(\eta_{E X P}\right)$ values for final-prototype $70 \mathrm{xz} \_28 \mathrm{y} . \ldots \ldots \ldots . . .88$

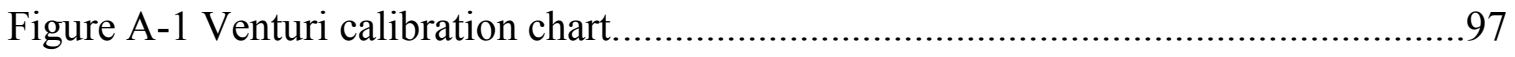

Figure B-1 $2036024 B$ motor data sheet. ............................................................99

Figure C-1 Blade dimensions (mm) for 70xz_28y final prototype............................... 100

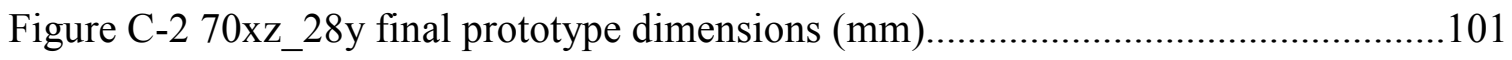




\section{NOMENCLATURE, ABBREVIATIONS, AND UNITS}

\section{Nomenclature}

A

$\beta$

$\beta_{1}$

$\beta_{2}$

$b$

$b_{2}$

$d$

$D_{2}$

$d_{2, \text { plate }}$

$d_{1}$

$d_{1, \text { plate }}$

$d_{\text {in }}$

$D_{1}$

$\delta$

$\delta_{\text {shroud }}$

$\delta_{\text {hub }}$

$\delta_{\text {inlet }}$

$\eta$ or $\eta_{\text {overall }}$

$\eta_{\text {blower }}$
Area symbol.

Angle of the relative velocity vector or impeller blade in the plane of the velocity diagram from the tangential direction.

Blade angle at inlet.

Blade angle at exit.

Width of impeller or other blade passage in the meridional direction.

Impeller blade width at the trailing edge.

Diameter of impeller at a general point.

Impeller diameter at exit.

Front plate diameter at the shroud exit of the

impeller $\left(d_{2, \text { plate }}-D_{2}\right) / 2=\delta_{\text {shroud }}$.

Impeller diameter at inlet.

Front plate diameter at impeller inlet. $\left(d_{1, \text { plate }}-d_{1}\right) / 2=\delta_{\text {inlet }}$.

Diameter of machined impeller at the inlet; includes the inlet lip dimension

Venturi diameter at the throat.

Clearance at various points, subscripted.

Tip clearance at the impeller shroud.

Tip clearance at the impeller exit hub.

Tip clearance at the impeller inlet $\left(d_{1, \text { plate }}-d_{1}\right) / 2=\delta_{\text {inlet }}$.

Overall blower efficiency value; represents the ratio of total head produced by the impeller to impeller shaft power $\left(\dot{W}_{S}\right)$.

Efficiency value from experiments. It is the ratio of static head 
measured to the calculated shaft power input $\left(\dot{W}_{S}\right)$.

$\eta_{E X P}$

Efficiency value from experiments. Represents the ratio of static pressure head measured to the electrical power input at the motor given $\left(\dot{W}_{\text {elec }}\right)$.

$\eta_{F L U}$

FLUENT efficiency. Represents the ratio of static head as given by FLUENT, to the impeller power calculated by $\operatorname{FLUENT}\left(\dot{W}_{I}\right)$.

$\eta_{H Y}$ Hydraulic efficiency value for blower. Represents the ratio of total head produced by the blower to the power given to the impeller $\left(\dot{W}_{I}\right)$.

$\eta_{H Y, F L U} \quad$ Hydraulic efficiency value calculated using FLUENT.

$\eta_{m} \quad$ Mechanical efficiency of the blower.

$\eta_{\text {motor }} \quad$ Efficiency of the motor used to drive the blower.

$\eta_{v} \quad$ Volumetric efficiency for the impeller.

$g \quad$ Acceleration due to gravity $\left(9.80665 \mathrm{~m} / \mathrm{s}^{2}\right)$.

$\left\{g_{p}\right\} \quad$ Set of fluid properties associated with gas handling phenomenon.

$h$

Static enthalpy.

$H \quad$ Head of air column; can also have the same meaning as the change in head $\Delta H_{t o t}$.

$\Delta H_{i} \quad$ The ideal head generated for an infinite number of blades that produces no blockage and no separation.

$\sum H_{L}$ All losses in the main flow passages from pump inlet to pump outlet.

$\Delta H_{\text {stat }} \quad$ Head change produced due to static pressure rise.

$\Delta H_{\text {tot }} \quad$ Change in head across blower stage, also called the "total dynamic head". 


$\begin{array}{ll}\Delta H_{v e l} & \text { Head change produced to velocity also called "dynamic head". } \\ l & \text { Blade, vane or passage arc length. } \\ \left\{l_{i}\right\} & \text { Infinite set of lengths that characterize blower geometry. } \\ I & \text { Current drawn by motor. } \\ \dot{m} & \text { Mass flow rate that is measured experimentally using the } \\ \dot{m}_{L} & \text { venturi. } \\ N & \text { Leakage mass flow rate, it is not measured. } \\ N_{S} \text { or } N_{S,(U S)} & \text { Rotational speed of the impeller. } \\ \Omega & \text { Specific speed in units of rpm, GPMUS, and ft. } \\ \Omega_{s} & \text { Angular velocity of blower. } \\ P_{s t a t} & \text { Non-dimensional specific speed. } \\ P_{t o t} & \text { Static pressure at a point. } \\ P_{v e l} & \text { Total pressure at a point. } \\ Q & \text { Dynamic pressure at a point. } \\ Q_{L} & \text { Volume flow rate or, more conveniently, "flow rate". } \\ Q_{s} & \text { Loss volume flow rate. } \\ \rho_{\text {air }} & \text { Flow coefficient. } \\ r & \text { Density of air. } \\ R_{1} & \text { Radial distance from axis of rotation. } \\ R_{2} & \text { Impeller radius at inlet. } \\ r_{e} & \text { Impeller radius at the blade trailing edge i.e. impeller exit. } \\ \operatorname{Re} & \text { Maximum value of } r \text { within the impeller inlet plane. } \\ \{S\} & \text { Machine Reynolds number. } \\ \tau_{w} & \text { Set of flow properties associated with solids in the pumpage. } \\ & \text { Cavitation coefficient. } \\ & \text { Shear stress at the wall. } \\ & \end{array}$




\begin{tabular}{ll}
$T$ & Torque. \\
$u$ & Internal energy of the fluid. \\
$u_{\tau}$ & Friction velocity at a point. \\
$U$ & Tangential speed $\Omega r$ of the point on the impeller at radius $r$ \\
$\mu$ & Absolute viscosity for air. \\
$V$ & Absolute velocity; also voltage supplied to power supply. \\
$v$ & Kinematic viscosity. \\
$W$ & Velocity of fluid relative to rotating impeller. \\
$X$ or $x$ & One of the two in-plane directions. \\
$Y$ or $y$ & Axial direction of the impeller. \\
$Z$ or $z$ & Elevation coordinates, also one of the two in-plane directions. \\
$Z_{i}$ & Number of impeller blades. \\
$\psi$ & Head coefficient. \\
$\{2-p h\}$ & Set of fluid properties associated with two phase flow. \\
$\dot{W}_{D}$ & Power utilized to overcome disk friction losses. \\
$\dot{W}_{\text {elec }}$ & Electrical power supplied to the motor at the main terminals. \\
$\dot{W}_{h y d r a u l i c}$ & Hydraulic power generated by blower $\left(=Q . P_{\text {stat }}\right)$ \\
$\dot{W}_{i}$ & Impeller power input for an ideal blower. \\
$\dot{W}_{I}$ & Power delivered to all fluid flowing through the impeller. \\
$\dot{W}_{S}$ & Shaft power. \\
$\dot{W}_{S}$ & Coefficient for shaft power. \\
$y_{P}$ & Distance from point $P$ to the wall. \\
\hline
\end{tabular}

\section{Abbreviations}

$\mathrm{AF}$

ATL
Airfoil Blade.

Aerosol Technology Laboratory at Texas A\&M

University. 
BC

BEP

BI

CAD

CFD

CSVI

D.C.

FC

HVS

JBPDS

NASA

$\mathrm{NPSH}$

Q3D

$\mathrm{RB}$

RP

RT

SLA

\section{Units}

Amp

${ }^{\circ} \mathrm{F}$

${ }^{\circ} \mathrm{R}$

$\mathrm{Ft}$

gpm

$1 "$

$\mathrm{kg} / \mathrm{s}$

$\mathrm{L} / \mathrm{min}$

$\mathrm{m}$

$\mathrm{m} / \mathrm{s}$
Backward Curved Blade.

Best Efficiency Point.

Backward Inclined Blade.

Computer Aided Design.

Computational Fluid Dynamics.

Circumferential Slot Virtual Impactor.

Direct Current.

Forward Curved Blade.

Hand-held Vacuum System.

Joint Biological Point Detection System.

National Aeronautics and Space Administration

Net Positive Suction Head.

Quasi Three Dimensional.

Radial Blade.

Rapid Prototyping.

Radial Tip Blade.

Stereolithography.

Ampere.

degree Fahrenheit.

degree Rankine.

feet.

gallon per minute.

inch.

kilogram per second

liter per minute.

meter.

meter per second. 


$\begin{array}{ll}\mathrm{Pa} & \text { Pascal. } \\ \mathrm{psi} & \text { pound pressure per square inch. } \\ \mathrm{lbm} / \mathrm{min} & \text { pound mass per minute. } \\ \mathrm{rpm} & \text { revolution per minute. } \\ \mathrm{rad} / \mathrm{s} & \text { radians per second. } \\ \mathrm{GPM} & \text { United States gallon per minute. } \\ \mathrm{V} & \text { Volts. } \\ \mathrm{W} & \text { Watt. } \\ \text { "WC } & \text { inches of water column (unit of pressure). }\end{array}$




\section{INTRODUCTION}

With the advent of chemical and biological weapons, bioterrorism is a major threat to the national security of the United States of America. The capability for real-time detection of airborne pathogens and toxins is necessary for the protection of military personnel and critical public environments (e.g., subways, sporting events, government buildings). Further, with the ever growing concern about environmental abuse, and the growing demand for pollution control, detection of air borne pollutants is also becoming critical.

Because of the perceived military threat and the growing demand for pollution monitoring, devices for near real-time detection and identification of airborne pathogens have been developed. The operating principle for current bio-aerosol detection devices is simple. The way most of these systems work, is to draw in a large quantity of air along with the particles as a sample. The particles contained in the sample need to be concentrated into a smaller stream for detection. At the current technology level, most of the airborne particle detection devices are bulky - weigh several hundred pounds, require trailer-transportation, and consume large amounts of energy. A valid case is the Joint Biological Point Detection System (JBPDS) being developed for the army (Black 2002). For the JBPDS, the detection system plus the electrical generator weight is approximately $530 \mathrm{lbs}(\sim 230 \mathrm{~kg})$ and it is transported on a truck (General Dynamics Armament and Technical Products: JBPDS, 2004). It has been progressively felt that in the near future the focus would shift towards smaller, lighter, and low power consuming airborne particle detection systems.

Future portable bio-aerosol samplers may utilize Circumferential Slot Virtual Impactors (CSVI) for concentration (Haglund et al. 2004). This would considerably reduce the

This thesis follows the style and format of Aerosol Science and Technology. 
power consumption of a biological detection unit (Isaguirre 2004). CSVIs typically operate in a flow range of $100 \mathrm{~L} / \mathrm{min}$ and require approximately $1000 \mathrm{~Pa}$ of pressure drop across them. Air is pulled in with a blower, which for future portable systems will need to be high-efficiency. Designing such blowers is a challenging area because of the dearth of commercially available blowers that operate at such low flow rates and low pressure rise values. Further, because effective utilization of power is necessary, especially for military field applications, a high efficiency blower will greatly increase the continuous operation time of battery-powered detection units.

A small cautionary note regarding the use of term 'low' for a pressure rise value of 1000 $\mathrm{Pa}$ : For most axial fans, a pressure rise value of $1000 \mathrm{~Pa}$ will fall in the range of mediumto-high, when compared with the normal design value for such fans. For centrifugal fans, on the other hand, this value is in the low range. At this point, the use of the term 'low' is general, and not intended to specify a preference for centrifugal blowers.

Development of such a state-of-the-art blower to meet the flow rate, pressure rise, and high efficiency requirements as set by the future aerosol applications is the goal of this study. The approach employed is to use computational fluid dynamics (CFD) as an extensive design tool. In the past, the design of turbo-machinery impellers has been based on empiricism (Tallgren et al. 2004). It is desired that through this study, the usefulness of the CFD based design approach for rotating-equipment design can be established. In the absence of literature for low flow rate and low pressure rise impellers, it is intended to create meaningful inroads and establish certain guidelines for designing such blowers. This will be accomplished by demonstrating that the coupled knowledge from CFD simulations and experiments complement each other in upgrading the operational efficiency of the current system and support the development of innovative designs. The tools required are knowledge about blowers (rotor-dynamics), fluid-dynamics, and experimental verification. 
The thesis follows a natural design process framework. First, the design goals, in terms of the flow rate, pressure-rise, and efficiency values are identified. Then an understanding of the different impeller types and the governing equations describing the energy transfer process are established. After this, the selection process to choose a blower type, pertinent to the design goals, is outlined. Next, a base blower design is selected and its performance simulated using CFD; this allows zeroing in on the optimum size that meets the set design goals. The optimum blower size is fabricated and evaluated on a test-rig to validate its performance. The other sections such as conclusion, recommendations, and appendices follow. 


\section{THEORY AND DESIGN}

\section{Establishing the System Requirements}

The main objective of this study is to design, develop, and performance-test a blower for future portable aerosol sampling applications. Designing a blower for a specific application is a daunting task requiring a fundamental understanding of fluid-mechanics, thermodynamics, structural mechanics, and also the marketplace economic requirements. Even before one starts the journey, it is necessary to specify the goal or the design requirements. A top-down approach has been adopted for the design process and, accordingly, the first step (Figure 1) entails establishing the system requirements that the blower will meet. This includes specifying the flow rate and the pressure rise that is required from the impeller, keeping in mind the trend towards future portable aerosol samplers. The blower will be designed for:

- Flow rate $(Q) \approx 100 \mathrm{~L} / \min \left(1.67 \times 10^{-3} \mathrm{~m}^{3} / \mathrm{s}\right)$

- $\quad$ Static pressure rise $\left(P_{\text {stat }}\right) \approx 4$ " WC $(1000 \mathrm{~Pa})$

- Shaft power consumption $\left(\dot{W}_{S}\right) \approx 4-5 \mathrm{~W}$ 


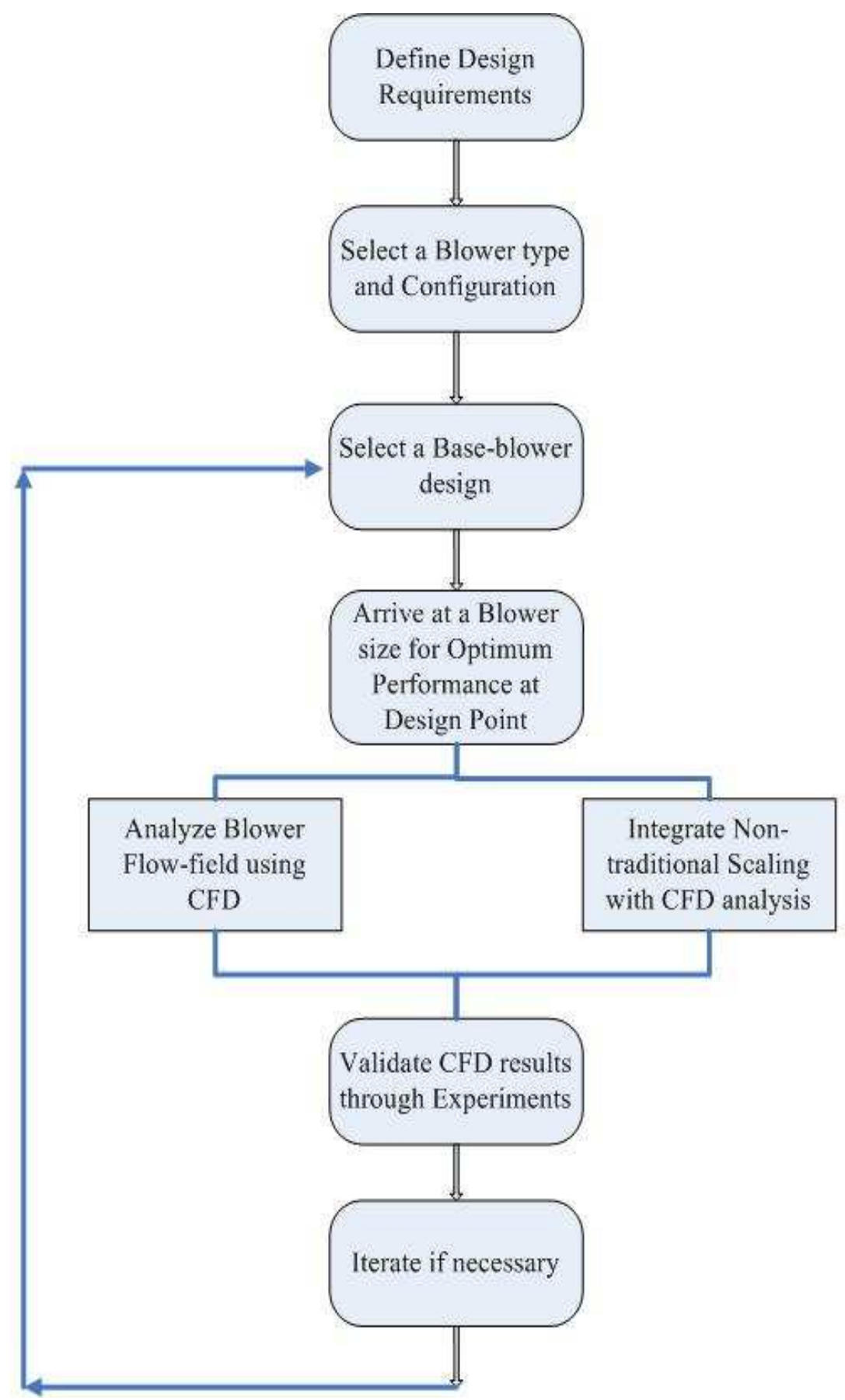

Figure 1: Top-down design approach framework. 


\section{Blower Type Selection}

Having established the design requirements, the next step is to decide upon the blower type. This is a critical question as the performance of the overall system depends on using the right pump-type for the right-application. The two broad classes of pumps are (Japikse et al. 1997): turbo-machinery (rotating) class, and the positive displacement class. At this point it is important to distinguish between the two - if the work done is readily described by force, $F$, times distance, then it is a positive displacement pump working by applying a force through a prescribed distance. By contrast, if the energy transfer is described by the torque times the angular velocity then it is a turbomachine. Further, within the turbo-machinery class there are the two extreme types: centrifugal and axial. Centrifugal pumps produce a large head rise since the work input, and the consequent head rise, is proportional to the impeller exit tangential speed squared $\left(U^{2}\right)$. If the rotational rate is constant, the head rise is also proportional to the square of exit radius $\left(R_{2}{ }^{2}\right)$. The axial pump, lacking this attribute, achieves less head rise, but can have large inlet area and hence can achieve very high flow rates (Figure 2). Before a decision can be made as to which blower type would be most applicable to meet the design requirements, one need to have insight into the selection methodology. This is outlined in the next four sections.

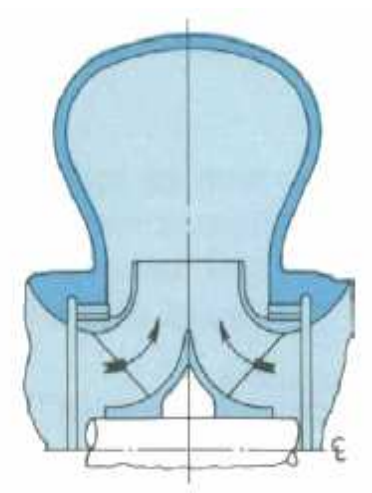

(a)

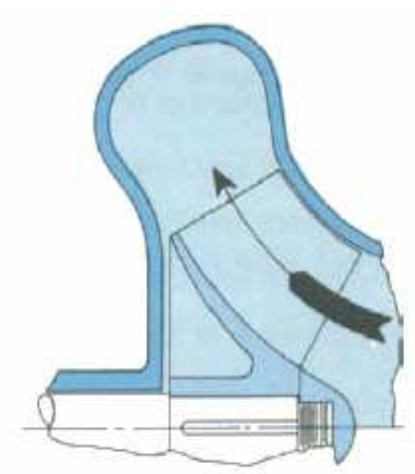

(b)

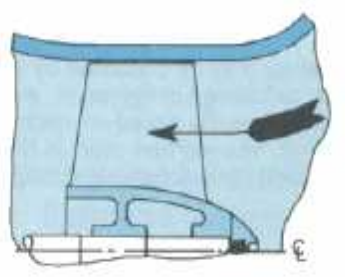

(c)

Figure 2: Cross-sections of different pumps: (a) Radial flow (b) Mixed flow (c) Axial flow (Hydraulics Institute). 


\section{Scaling and Similitude}

Scaling and non-dimensional study of the equations governing the performance of a system is a powerful tool. Scaling is important because if one has the set of characteristic curves for a given pump, then that machine can be used as a 'model' to satisfy similar conditions of service at different speed and a different size ${ }^{1}$. Blower selection, design, and performance are influenced primarily by fluid dynamics, represented by the velocity components at the various spatial points. In the literature different notations are used to define the velocity components. To avoid any confusion, the notation used to define the velocity components is explained below. Throughout the thesis the NASA system of capital letters $U, V$, and $W$ is employed where:

$$
\begin{aligned}
& U=\text { tangential speed } \Omega r \text { of the point on the impeller at radius } r, \mathrm{~m} / \mathrm{s} \\
& V=\text { absolute velocity of the fluid, } \mathrm{m} / \mathrm{s} \\
& W=\text { velocity of fluid relative to rotating impeller, } \mathrm{m} / \mathrm{s}
\end{aligned}
$$

Scaling a given geometry to a new size means multiplying every linear dimension of the model by the scale factor, including all clearances and surface roughness elements. The performance of the model is then scaled to correspond to the scaled-up model by requiring similar velocity diagrams and assuming that the influences of fluid viscosity and vaporization (for pumps handling liquids) are negligible. Equations 1, 3, and 6 illustrate this. The blade velocity $U$ (Equation 4) varies directly with rotational speed $N$ or angular speed $\Omega$ - and directly with size, as expressed by the radius $r$. For the fluid velocity $\mathrm{V}$ (or $\mathrm{W}$ ) to be in proportion to $U$, the flow rate $Q$ must therefore vary as $\Omega r^{3}$ (Karassik et al. 2000); hence, the "specific flow" $Q_{s}$ must be constant (Equation 2). Further, as the total head $\left(\Delta H_{t o t}\right)$ is the product of two velocities, it must vary as $\Omega^{2} r^{2}$;

\footnotetext{
${ }^{1}$ A cautionary note to be kept in mind - Strictly speaking the term pump is reserved for devices that use liquid e.g. water pumps. In cases where one deals with air, using the term blower would be more appropriate. Unfortunately, not much information is available for designing low flow rate, and low pressure rise blowers. For the present application, since the air-flow is incompressible, the best approach is to rely on the knowledge accrued by the pump industry. Since most of the material has been borrowed from pump literature, the terms pump, impeller, blower have been used interchangeably throughout the text
} 
hence, the head coefficient $\psi$ must be constant (Equation 5). Finally, as power is the product of pressure-rise and flow rate, shaft power $\dot{W}_{S}$ must vary as $\rho \Omega^{3} r^{5}$; hence, the power coefficient must be constant (Equation 7) (Karassik et al. 2000).

$$
\begin{aligned}
& Q=A V\left\{\begin{array}{l}
A \alpha \mathrm{R}_{2}^{2} \alpha D^{2} \\
V \alpha \Omega R_{2} \alpha N D
\end{array}\right. \\
& \Rightarrow Q \alpha N D^{3} \text { or } \Omega R_{2}^{3} \\
& \text { and } \quad \frac{Q}{\Omega R_{2}{ }^{3}}=\text { Constant }=Q_{s} \\
& g \Delta H_{\text {tot }}=\eta_{H Y} \Delta\left(U V_{\theta}\right) \\
& \Rightarrow \Delta H_{\text {tot }} \alpha N^{2} D^{2} \\
& U=\Omega r \\
& \psi=\frac{g \Delta H_{\text {tot }}}{\left(\Omega R_{2}\right)^{2}}=\text { Constant } \\
& \dot{W}_{S}=\frac{\rho Q g \Delta H_{\text {tot }}}{\eta_{\text {overall }}} \\
& \Rightarrow \dot{W}_{S} \alpha N^{3} D^{5} \\
& \hat{\dot{W}}_{S}=\frac{\dot{W}_{S}}{\rho \Omega^{3} R_{2}{ }^{5}}=\text { Constant }
\end{aligned}
$$

Uniform scaling in pump geometry or shape produces a new set of curves - shaped differently but similar to each other. Similitude enables a designer to work from a single dimensionless set of performance curves for a given pump model. This is a practical, but a special, case of the more general statement. In general the performance of a pump, as represented by efficiency, total head, and shaft power, is expressed in terms of the complete physical equation as follows (Karassik et al. 2000):

$$
\eta_{\text {overall }}, \Delta H_{\text {tot }}, \dot{W}_{S}=f c t^{\prime} s .\left(Q, R_{2}, \Omega, \rho, v, N P S H,\{2-p h\},\left\{g_{p}\right\},\{S\},\left\{l_{i}\right\}\right)
$$


where $\left\{l_{i}\right\}$ is the infinite set of lengths that defines the pump stage geometry. A common group of these lengths is illustrated in Figure 3.

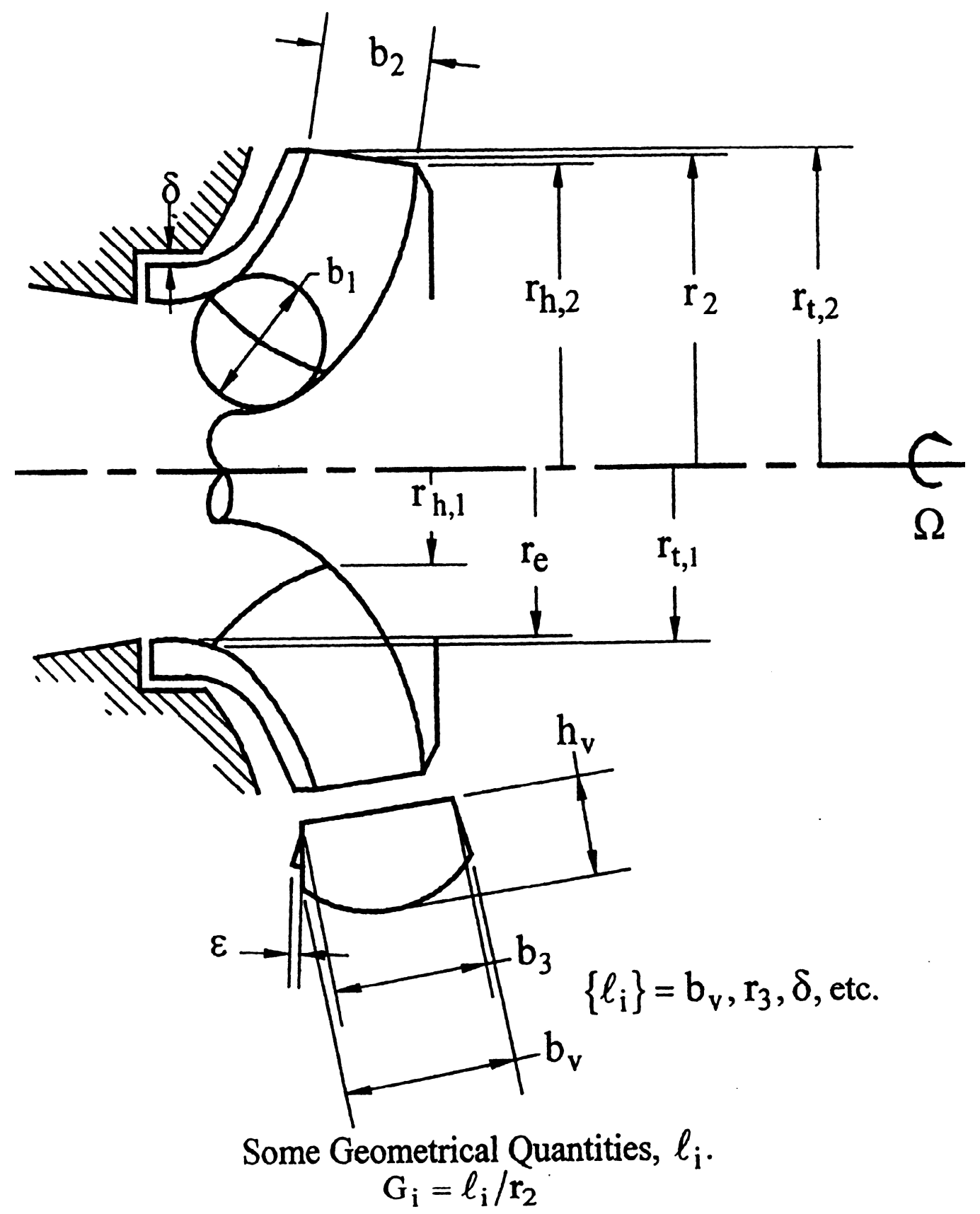

Figure 3: Defining the geometry of a pump stage (Karassik et al. 2000, reprinted with permission of McGraw-Hill). 
Non-dimensionally, Equation 8 can be expressed as:

$$
\eta_{\text {overall }}, \psi, \hat{W}_{S}=f c t^{\prime} s .\left(Q_{s}, \operatorname{Re}, \tau_{2},\{2-\Phi\},\left\{\Gamma_{p}\right\},\{\Sigma\},\left\{G_{i}\right\}\right)
$$

where the dimensionless quantities containing flow rate, viscosity and NPSH are respectively defined as follows:

$$
\begin{aligned}
Q_{s} & =\frac{Q}{\Omega R_{2}{ }^{2}} \text { Specific Flow } \\
\operatorname{Re} & =\frac{\Omega R_{2}^{2}}{v} \text { Machine Reynold Number } \\
\tau_{2} & =\frac{2 g N P S H}{\Omega^{2} R_{2}^{2}} \text { Cavitation Factor }
\end{aligned}
$$

- $\left\{G_{i}\right\}=\left\{l_{i} / R_{2}\right\}$ defines the dimensionless geometry or shape

- $\{2-\Phi\}=$ the dimensionless quantities arising from the set of fluid, thermal, vaporization, and hear transfer properties $\{2-p h\}$ that influence the flow of twophase vapor and liquid.

- $\{\Sigma\}=$ the dimensionless quantities arising from the set of properties associated with entrained solids and emulsifying fluids that affect the performance of slurry pumps and emulsion pumps.

Equation 9 represents the most general treatment for describing the performance of an impeller. For the present design, since one is dealing with air, the factors corresponding to two-phase flow $(2-\Phi)$, entrained and emulsifying fluids $\{\Sigma\}$, and the cavitation factor $\tau_{2}$, are eliminated. Hence:

$$
\eta_{\text {overall }}, \psi, \hat{\dot{W}}_{S}=f c t^{\prime} s .\left(Q_{s}, \operatorname{Re},\left\{G_{i}\right\}\right)
$$

Equation 11 states that if we know all the quantities on the right hand side, then the efficiency, head, and power characteristics are fixed. Therefore, pump performance is a function of pump geometry, flow rate, and the machine Reynolds number. 


\section{Specific Speed and Optimum Geometry}

Ideally, the hydraulic geometry or shape of a pump stage can be chosen for given values of the other independent variables in Equation 11 to optimize the resulting performance. One restricts the operational range of the pump by imposing certain constraints on the head and power. Two such conditions that are common are

a) no positive slope allowed anywhere along the $\Delta H_{\text {tot }}-v s .-\Delta Q$ curve (this ensures stable pump performance (Tuzson 2000)).

b) the peak power consumption must occur at the best efficiency point (BEP) (often called the "non-overloading" condition)

This means that by specifying the flow-coefficient, the operational machine Reynolds number, and the head and power coefficients, Equation 11 can be used to generate the set of geometric lengths that maximizes the best efficiency $\eta_{B E P}$. This is the direct design approach.

This direct design approach is rarely practical to implement. One simplifies Equation 11 using certain assumptions, and this will lead to a single dimensionless number, specific speed $\left(\Omega_{s}\right)$, a well-known figure of merit, that characterizes the geometry of fluid machinery. One assumes that a typical pumping situation involves:

- Negligible influence of viscosity as long as the flow is principally in the turbulent regime (a frequent situation for most, but not all, pumps (Japikse et al. 1997, Karassik et al. 2000). Pump performance will change with Reynolds number to a small power of approximately 0.15 to 0.2 (Japikse et al. 1997). This effect is usually set aside for later correction or ignored completely.

- the geometry can be represented by a single characteristic size $R_{2}$ (impeller blade tip radius). 
In this situation, Equation 11 is left with one significant independent variable; namely, the specific flow $Q_{s}$. One does not know the size of the pump stage a priori; so, $R_{2}$ is eliminated by replacing $Q_{s}$ in Equation 11 with a new quantity that is the result of dividing the square root of $Q_{s}$ by the $3 / 4$-power of the head coefficient $\psi$. Thus, from the definitions just given, we arrive at the specific speed $\Omega_{s}$ as the independent variable in terms of which the geometry is optimized (Sabersky et al. 1966).

$$
\Omega_{s}=\frac{\Omega \sqrt{Q}}{\left(g \Delta H_{t o t}\right)^{3 / 4}}=\frac{\sqrt{Q_{s}}}{\psi^{3 / 4}}
$$

For convenience, specific speed is usually expressed in terms of the conventional quantities, for example, the form found in the United States and its relationship to $\Omega_{s}$ is as follows:

$$
\Omega_{s}=\frac{N(\mathrm{rpm}) \sqrt{Q\left(\mathrm{GPM}_{\mathrm{US}}\right)} /\left[\Delta H_{t o t}(\mathrm{ft})\right]^{3 / 4}}{2733.016}=\frac{N_{s,(U S)}}{2733.016}
$$

\section{Rotor Shape as a Function of Specific Speed}

Optimization of pump hydraulic geometry in terms of best efficiency point (BEP) specific speed has taken place empirically and analytically throughout the history of pump development. An approximate illustration of the results of this process for pump rotors or impellers is shown in Figure 4. Not only does the geometry emerge from the optimization process but also the head, flow, and power coefficients for each shape as well. Figure 4 also shows the approximate values for the optimum BEP head coefficient $\psi$. As can be seen, the specific speed of the application suggests the most efficient configuration: centrifugal, mixed flow, or axial turbomachines, depending on increasing specific speed (Tuzson 2000). Since specific speed is based on fundamental 
physical principles (Tuzson 2000), and not an arbitrary classification, it can also be used to characterize the operational range of positive displacement pumps (Balje 1962, Cartwright 1977). Figure 5 further refines the choice of impeller profiles for a given specific speed.

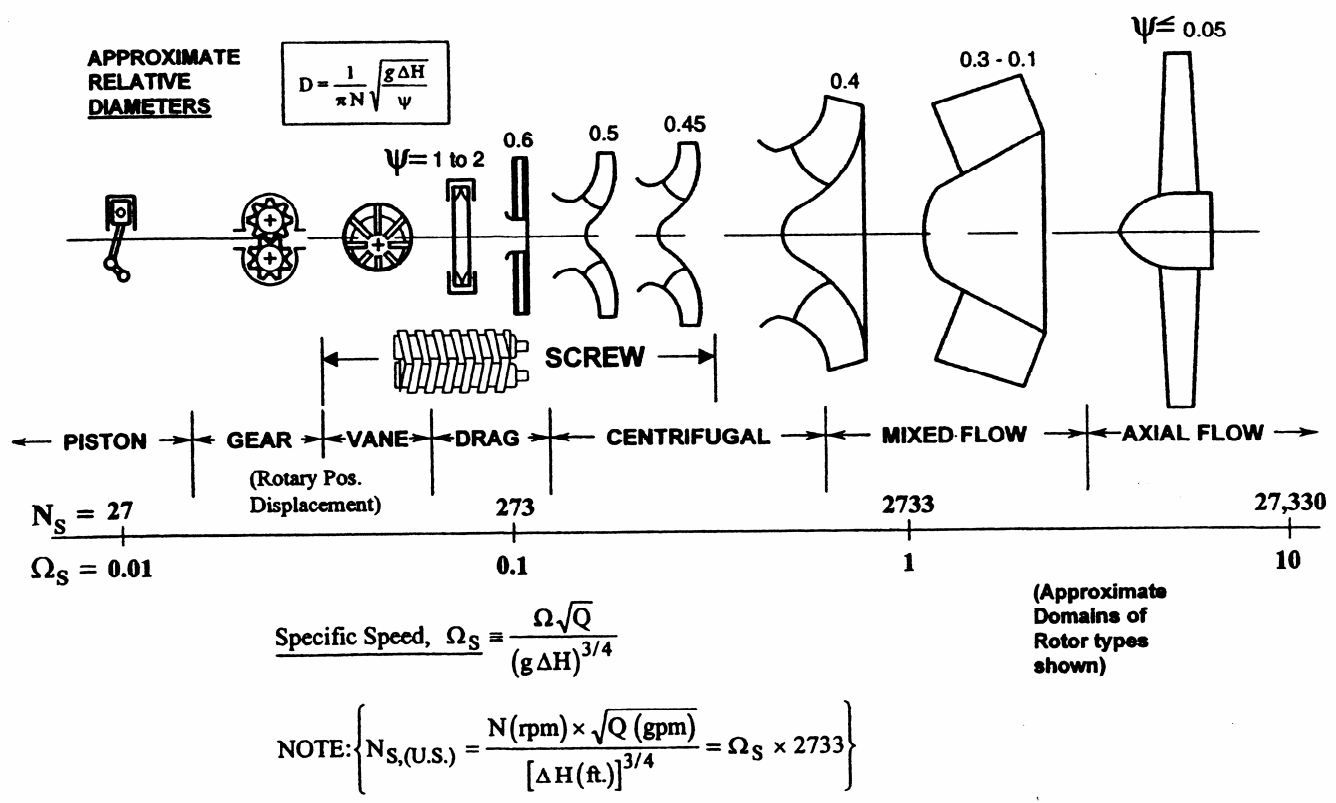

Figure 4: Optimum geometry as a function of BEP specific speed (Karassik et al. 2000, reprinted with permission of McGraw-Hill).

Values of specific speeds

(Single suction)

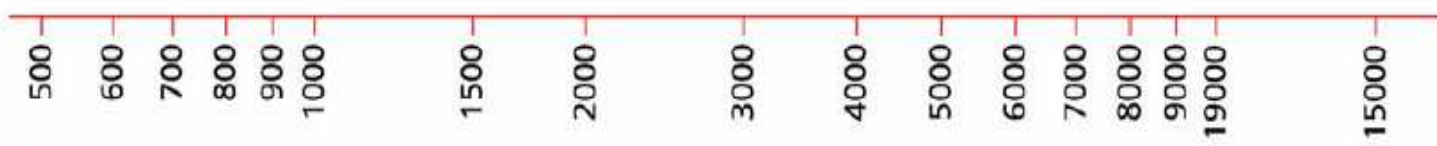

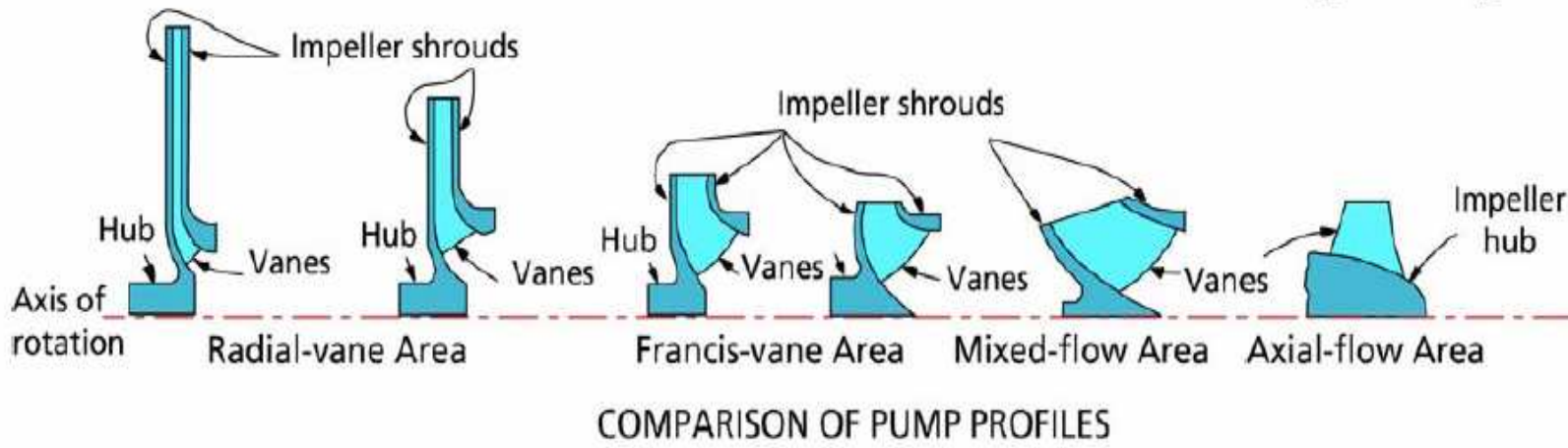

Figure 5: Specific speed values for different pump designs (Hydraulics Institute). 


\section{Selection of Optimum Specific Speed Values}

There are two possible methods to vary the specific speed. The objective in both is to arrive at a specific speed value that maximizes the overall efficiency $\left(\eta_{\text {overall }}\right)$ (Figure 6). Generally the design applications specify the flow rate and the head-rise values, leaving the shaft speed open. Efficiency can be improved by choosing a shaft speed that changes the specific speed towards an optimal value (Tuzson 2000). This is the first approach. Another possibility is reducing the head requirement per stage by using multiple stages. This allows the selection of an optimum specific speed value per stage. A similar approach can be accomplished by reducing the total volume flow rate through an impeller stage by using multi-suction blowers. This is done when size restrictions apply. This won't be applicable in the present case as reducing the flow rate, while maintaining the same head-requirement, reduces the specific speed value and hence the efficiency.

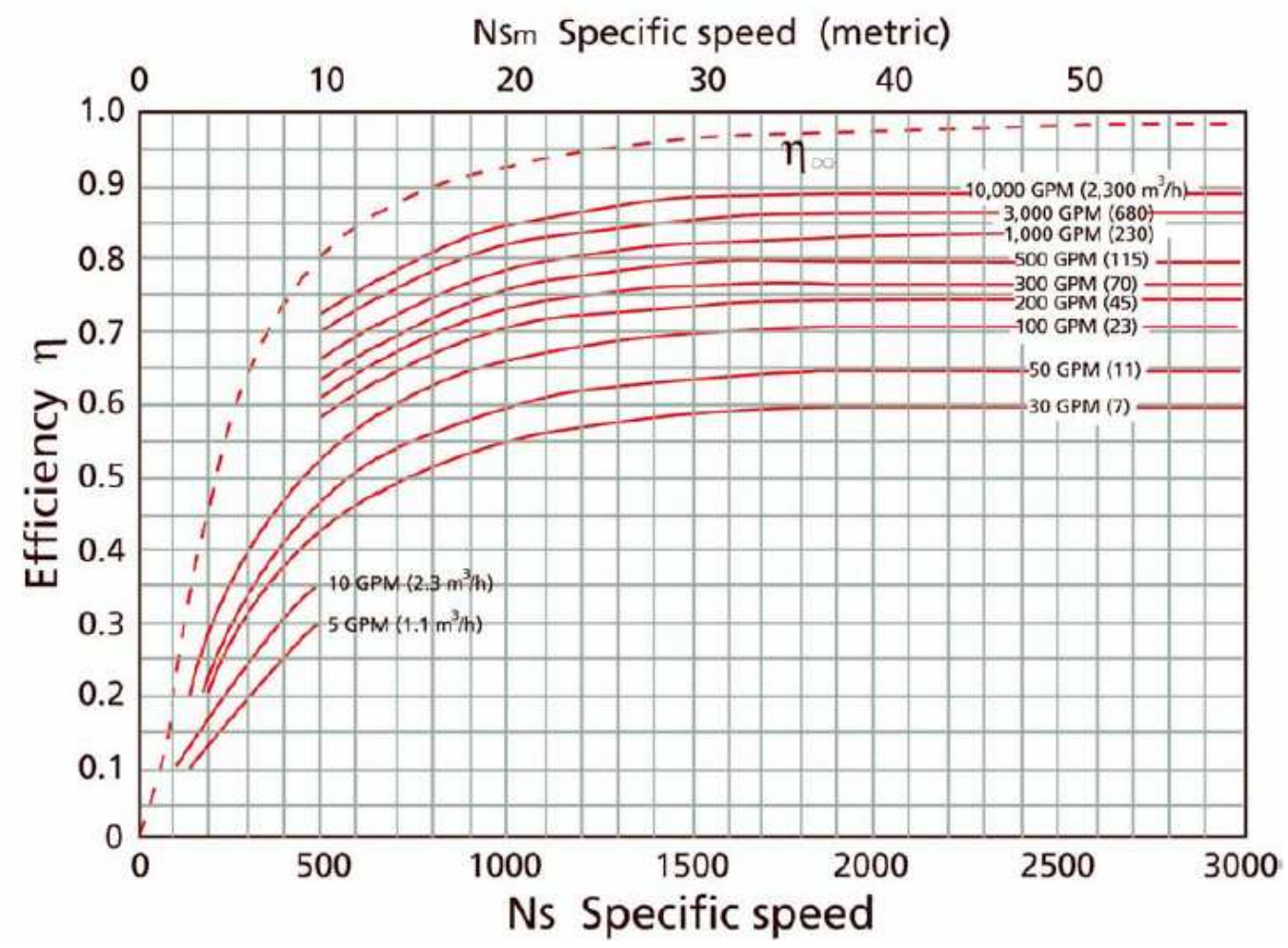

Figure 6: Efficiency values for pump with different specific speeds (Karassik et al. 2000, reprinted with permission of McGraw-Hill). 
Even at this point, Method One, changing the rpm seems more feasible assuming that the pressure rise and the flow rate can be achieved. Designing the application for portable use also favors single stage. Nonetheless, both approaches are discussed in the section on sample calculation; but first one needs to develop the equations for estimating the head change.

\section{Estimating Head Rise $\left(\Delta H_{t o t}\right)$ across an Impeller}

To estimate the total head change across an impeller, the energy transfer equations across a general rotating impeller need to be developed. Hydraulics or fluid dynamics has the primary influence on the geometry of a rotordynamic pump stage. It is basic to the energy transfer or pumping process. Action of the mechanical input shaft power to affect an increase in the energy of fluid is governed by the First Law of Thermodynamics. Realization of that energy in terms of pump pressure rise of head involves losses and consequently the Second Law of Thermodynamics.

\section{First Law of Thermodynamics}

Fluid flow, whether liquid or gas, through a centrifugal pump is essentially adiabatic; heat transfer being negligible in comparison to the other forms of energy transfer process. Further, while the delivery of energy to fluid by rotating blades is inherently unsteady (varying pressure from blade to blade as viewed in an absolute reference frame), the flow across the boundaries of a control volume surrounding the pump is essentially steady, and the First Law of Thermodynamics for the pump can be expressed in the form of the adiabatic steady-flow energy equation (Equation 14) as follows:

$$
\dot{W}_{S}=\dot{m}\left[\left(h+\frac{V^{2}}{2}+g Z_{e}\right)_{\text {out }}-\left(h+\frac{V^{2}}{2}+g Z_{e}\right)_{\text {in }}\right]
$$




$$
\text { where } h=u+\frac{P_{\text {stat }}}{\rho}
$$

Here, shaft power $\dot{W}_{S}$ is transformed into fluid power, which is the mass flow rate $(\dot{m})$ times the change in the total enthalpy (which includes static enthalpy, velocity energy per unit mass, and potential energy due to elevation in a gravitational field that produces acceleration at rate $g$ ) from inlet to outlet of the control volume (Figure 7).

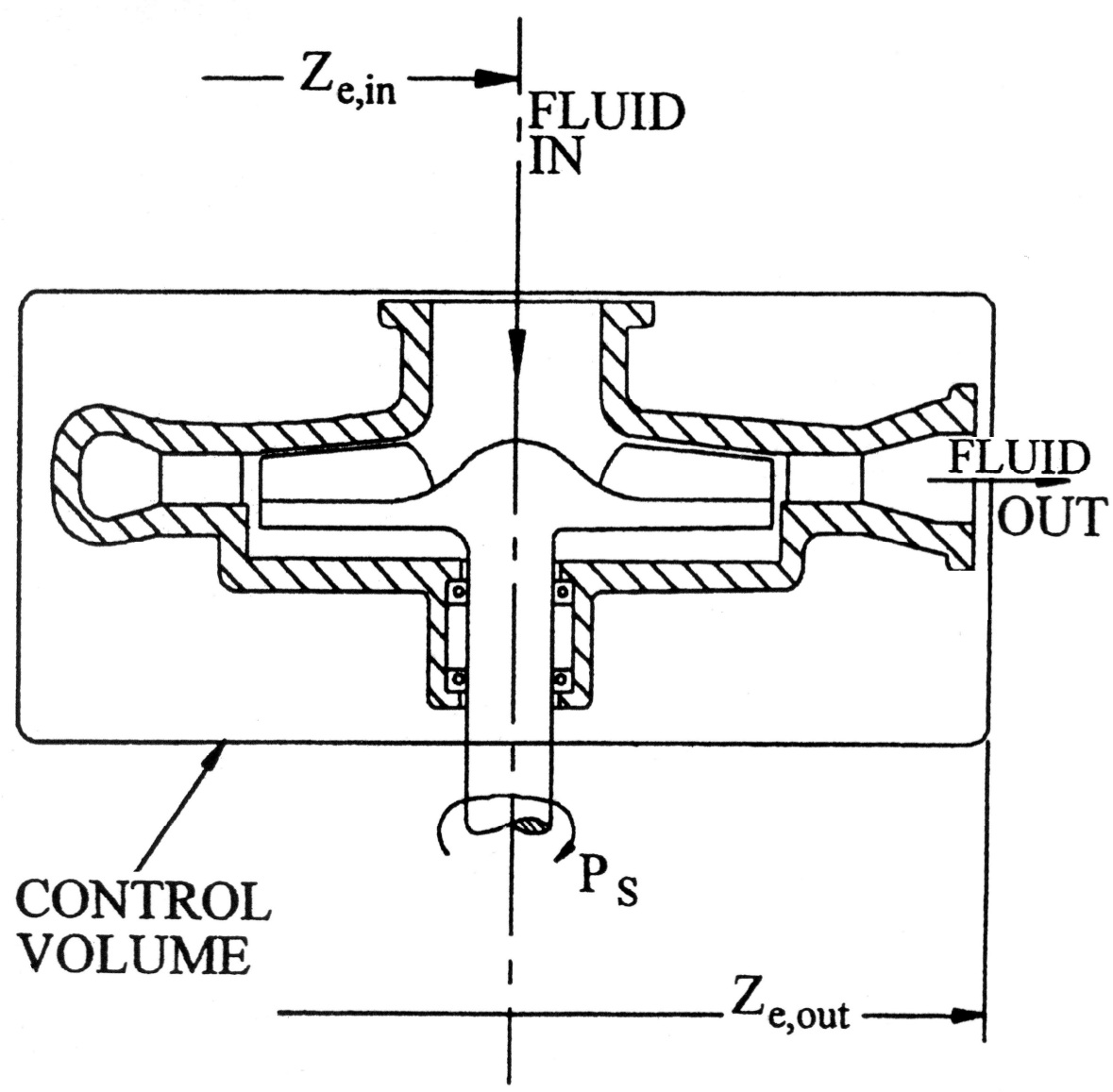

Figure 7: Energy balance across a control volume for a rotating impeller. 
For incompressible fluids, Equation 14 can be rearranged in terms of head, viz:

$$
\begin{aligned}
\frac{\dot{W}_{S}}{\dot{m}} & =g \Delta H_{t o t}+\Delta u \\
\text { where } \quad H_{t o t} & =\frac{P_{\text {stat }}}{\rho g}+\frac{V^{2}}{2 g}+Z_{e}
\end{aligned}
$$

The change in $H_{t o t}, \Delta H_{t o t}$, is called the "head" of the pump; and, because $H$ (Equation 15) includes the velocity head $V^{2} / 2 g$ and the elevation head $Z_{e}$ at the point of interest, $\Delta H_{t o t}$ is often called the "total dynamic head" (Karassik 2000)

\section{Sample Calculations for Impeller Specific Speed}

For calculation of specific speed, $N(\mathrm{rpm})$, the volume flow rate $(Q(U S g p m))$ and the total

head rise $\left(\Delta H_{t o t}(f t)\right)$ values are needed. The approach of selecting an impeller type can be summarized as follows:

- Choose a rpm value

- Estimate the head change

- Based on the specific speed value decide an impeller type, refer Figures 4 and 5

- Make sure that the specific speed corresponds to the point of maximum efficiency (Figure 6), if not, change the specific speed or use multiple stages

- Calculate the impeller size using the optimum BEP head coefficient $\psi$ given in Figure 4.

- Iterate if needed 


\section{Single Stage Impeller Calculations}

Sample calculation is given below. At this stage, the calculation of the velocity head is ignored, as the impeller size is not known. Instead one assumes that the effect of the exit velocity has been included in the pressure rise value that is used in the calculations. The following values are chosen:

- $\quad N=15000 \mathrm{rpm} \rightarrow \Omega \sim 1570 \mathrm{rad} / \mathrm{s}$

- $Q=100 \mathrm{~L} / \mathrm{min} \sim 26.4 \mathrm{GPM}_{\mathrm{US}}$

- $\Delta P_{\text {total }}=1000 \mathrm{~Pa}$

- $\rho=1.225 \mathrm{~kg} / \mathrm{m} 3$ (Bleier 1997)

- $\Delta Z_{e}=0$ (we are dealing with air, so elevation head is negligible compared with pressure and velocity heads)

Therefore,

$$
\Delta H_{\text {tot }} \approx \Delta H_{\text {stat }}=\frac{1000 \mathrm{~Pa}}{\left(1.225 \mathrm{~kg} / \mathrm{m}^{3}\right) \cdot\left(9.8 \mathrm{~m} / \mathrm{s}^{2}\right)}+0=83.3 \mathrm{~m}=273.3 \mathrm{ft}
$$

and,

$$
N_{s,(U S)}=\frac{N(\mathrm{rpm}) \sqrt{Q\left(\mathrm{GPM}_{\mathrm{US}}\right)}}{\left[\Delta H_{\text {tot }}(\mathrm{ft})\right]^{3 / 4}}=\frac{(15000 \mathrm{rpm}) \cdot\left(26.4 \mathrm{GPM}_{\mathrm{US}}\right)^{0.5}}{(273.3 \mathrm{ft})^{3 / 4}}=1147 \frac{{\mathrm{rpm} \cdot \mathrm{GPM}_{\mathrm{US}}}^{0.5}}{\mathrm{ft}^{0.75}}
$$

This value of specific speed corresponds to a centrifugal type impeller (Figure 4). The expected efficiency is about 0.58 (Figure 6). The efficiency value is not the maximum possible at this flow rate, which suggests that a higher rpm value could increase the efficiency. These points are further discussed in the next section, but before that, impeller calculations for a two stage impeller are given. 


\section{Two Stage Impeller Calculations}

For the two impeller model, all the variable values are kept the same. Only difference is in the head-rise value per stage. Assume that when two stages are employed, the head produced per-stage will be approximately half the total value (Karassik 2000). The following values are chosen for the two stage impeller calculations:

- $\quad N=15000 \mathrm{rpm} \rightarrow \Omega \sim 1570 \mathrm{rad} / \mathrm{s}$

- $Q=100 \mathrm{~L} / \mathrm{min} \sim 26.4 \mathrm{GPM}_{\mathrm{US}}$

- $\Delta P_{\text {total }}=1000 \mathrm{~Pa}$

- $\rho=1.225 \mathrm{~kg} / \mathrm{m} 3$ (Bleier 1997)

- $\Delta Z_{e}=0$

Therefore,

$$
\Delta H_{\text {tot, } \text { per-stage }}=\frac{1000 / 2 \mathrm{~Pa}}{\left(1.225 \mathrm{~kg} / \mathrm{m}^{3}\right) \cdot\left(9.8 \mathrm{~m} / \mathrm{s}^{2}\right)}+0=\frac{83.3}{2} \mathrm{~m}=137 \mathrm{ft}
$$

and,

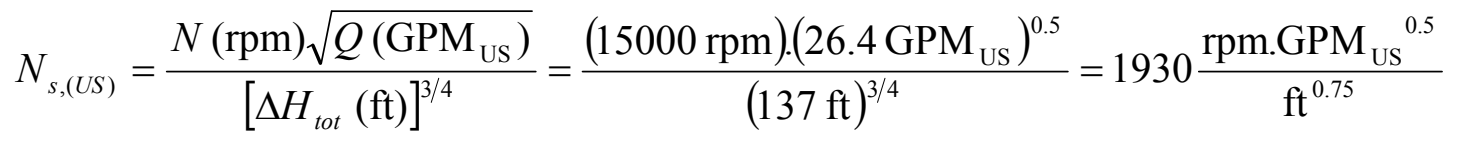

This corresponds to a mixed-flow impeller (Figure 4). Further, Figure 6 shows an expected efficiency value of 0.60 . This is not an appreciable difference when compared with the single stage centrifugal blower. Besides, using a higher rpm value with the single stage centrifugal blower can get us to this efficiency value.

Because the same performance level can be achieved with a single stage centrifugal blower, there is no reason in to use a two stage mixed flow impeller. Further, the two stage impellers will lead to portability challenges, and also rotordynamic balance problems. Also, a multi-stage axial fan will have inter-stage transfer losses that might 
reduce the performance. Therefore, a single stage centrifugal blower was selected as the design configuration.

\section{Size Calculation for Single Stage Centrifugal Blower}

Finally, the value of $R_{2}$ can be calculated using the value of specific speed obtained from Equation 18 and an approximate value of the optimum BEP head coefficient, $\psi$. From Figure 4, for a specific speed value of 1150 , the head coefficient value for optimum performance is 0.45 .

$$
\begin{aligned}
& \psi_{B E P} \sim 0.45 @ N_{s(U S)}=1150 \\
& \psi_{B E P}=\frac{g \cdot \Delta H_{t o t}}{\Omega^{2} R_{2}{ }^{2}} \\
& \Rightarrow R_{2}=\left(\frac{g \cdot \Delta H_{t o t}}{\Omega^{2} \cdot \psi_{B E P}}\right)^{0.5}=\left(\frac{\left(9.8 \mathrm{~m} / \mathrm{s}^{2}\right) \cdot(83.30 \mathrm{~m})}{\left(1570.8 \mathrm{rad} / \mathrm{s}^{2}\right)^{2} \cdot(0.45)}\right)^{0.5}=0.027 \mathrm{~m}=1.07 "
\end{aligned}
$$

\section{Single Stage Centrifugal Blower: Observations}

Having chosen a single stage centrifugal blower as the design configuration, important observations can be drawn from Equations 17, 18, and 21 along with Figures 4, 5, and 6. Figure 5 suggests selection of a Radial-vane area type centrifugal impeller. This will be an important point when selecting a base blower design in the next sections.

Figure 6 shows that the expected overall efficiency $\left(\eta_{\text {overall }}\right)$ is about 0.58 . This implies that even though the impeller will produce the static pressure rise at the desired flow rate, it won't be the most efficient design. At the desired flow rate of $26 \mathrm{GPM}_{\mathrm{US}}$ the most

efficient impeller designs have a specific speed $\left(N_{s,(U S)}\right)$ in the range of $1400-2000$. Therefore, increasing the rpm can add a few percentage points to the blower efficiency. 
By operating at a higher rpm the specific speed is increased and the impeller efficiency approaches a limiting value of 0.60 . Operating at a higher rpm value implies a smaller impeller size. Therefore, the trend points towards smaller size and higher rpm. Increasing the rpm value beyond a certain limit is not effective as the maximum attainable efficiency curve flattens out as the specific speed value is increased.

A combination of $15,000 \mathrm{rpm}$ and an impeller size $D_{2}=2.14$-inches will fulfill the requirements, but it won't be the best possible combination. Another important point to be considered is the value of the velocity head at the exit. At the exit, velocity is $V_{2} \sim \Omega R_{2}=42.41 \mathrm{~m} / \mathrm{s}$ (or less if centrifugal blower with backward curved (BC) blades is chosen (Karassik 2000)); implying that the magnitude of the velocity head $\left(V^{2} / 2 g\right)$ is $91.8 \mathrm{~m}$. This is of the same order as the overall pressure rise head; meaning that the total head produced by the impeller will be more than $83.30 \mathrm{~m}$. Hence, the actual specific speed value at 15,000 rpm will be lower than what was calculated in Equation 18. This also suggests using a higher rpm value than 15,000. But as a first approximation, the approach is adequate to help determine the impeller type and the range of the impeller size. Calculations for specific speed values and impeller sizes for different rpm values are given below in Table 1.

From the table it can be observed that all the specific-speed values fall within the range that corresponds to centrifugal impellers. Hence the impeller type is fixed: Centrifugal type blower and it is a single stage configuration. Combinations of rpm and impeller size that can be expected to show the best efficiency are shown as boldface in Table 1. Figure 6 further shows that beyond a specific speed value of $\sim 2000$, the efficiency does not change This implies that increasing the rpm beyond a certain value (say $~ 30,000$; after accounting for the velocity head) won't lead to any gain in efficiency. This point is further elaborated below. 
Table 1: Specific speed values and blower sizes at different rpms.

\begin{tabular}{|c|c|c|c|c|c|c|}
\hline RPM & $N_{s,(U S)}$ & $\begin{array}{c}\text { Impeller } \\
\text { Type }\end{array}$ & $\begin{array}{c}\psi_{B E P} \\
\text { (Figure 4) }\end{array}$ & $\mathrm{R}_{2}(\mathrm{~m})$ & $\begin{array}{c}\mathrm{R}_{2} \\
\text { (inch) }\end{array}$ & $\begin{array}{c}\mathrm{D}_{2} \\
\text { (inch) }\end{array}$ \\
\hline 5000 & 382 & Centrifugal & 0.6 & 0.070 & 2.8 & 5.6 \\
\hline 10000 & 764 & Centrifugal & 0.5 & 0.040 & 1.5 & 3.0 \\
\hline 15000 & 1147 & Centrifugal & 0.45 & 0.030 & 1.1 & 2.2 \\
\hline 20000 & $\mathbf{1 5 2 9}$ & Centrifugal & 0.45 & 0.020 & 0.8 & $\mathbf{1 . 6}$ \\
\hline 25000 & $\mathbf{1 9 1 1}$ & Centrifugal & 0.45 & 0.016 & 0.6 & $\mathbf{1 . 2}$ \\
\hline 30000 & $\mathbf{2 2 9 4}$ & Centrifugal & 0.4 & 0.014 & 0.6 & $\mathbf{1 . 2}$ \\
\hline 35000 & 2676 & Centrifugal & 0.4 & 0.012 & 0.5 & 1.0 \\
\hline 40000 & 3058 & Centrifugal & 0.4 & 0.011 & 0.4 & 0.8 \\
\hline
\end{tabular}

Before any conclusions are drawn, there are two more important ratios to be considered. One of them is the ratio of pressure to velocity-head, shown in Table 2. Second is the ratio of flow rate and rpm $(\mathrm{gpm} / \mathrm{rpm})$, shown in Figure 8.

Although the ideal value of pressure to velocity-head ratio is not mentioned in the literature, it must be kept low. This is because, for a given pressure head, if the velocityhead is increased, then the energy given by the impeller is being used to accelerate the air, which is discarded. Hence the high velocity head is not utilized. Even if one plans to recover the velocity head as static pressure - using a diffuser, the process will have low efficiency because of flow separation caused by adverse pressure gradients.

Further, from Figure 8, for a fixed flow rate, as the rpm is increased, the maximum attainable efficiency value is reduced. At an rpm of 20,000 the $(\mathrm{gpm} / \mathrm{rpm})$ ratio is 0.0013, which is just about the lower limit shown in Figure 8. Therefore, one would want to operate at the lowest possible rpm that ensures a high enough specific speed to get the maximum efficiency. 
Table 2: Ratio of pressure head and theoretical velocity head for different rpm and impeller diameter combinations.

\begin{tabular}{|c|c|c|c|c|c|}
\hline $\mathrm{RPM}$ & $\mathrm{R}_{2}(\mathrm{~m})$ & $\begin{array}{c}V_{2}\left(\sim \Omega R_{2}\right) \\
(\mathrm{m} / \mathrm{s})\end{array}$ & $\Delta H_{\text {vel }} \sim \frac{V_{2}^{2}}{2 g}$ & $\Delta H_{\text {stat }}$ & $\frac{\Delta H_{\text {stat }}}{\Delta H_{\text {vel }}}$ \\
\hline 5000 & 0.070 & 36.8 & 69.4 & 83.3 & 1.2 \\
\hline 10000 & 0.039 & 40.4 & 83.3 & 83.3 & 1.0 \\
\hline 15000 & 0.027 & 42.5 & 92.5 & 83.3 & 0.9 \\
\hline 20000 & 0.020 & 42.6 & 92.5 & 83.3 & 0.9 \\
\hline 25000 & 0.016 & 42.6 & 92.5 & 83.3 & 0.9 \\
\hline 30000 & 0.014 & 45.2 & 104.1 & 83.3 & 0.8 \\
\hline 35000 & 0.012 & 45.2 & 104.1 & 83.3 & 0.8 \\
\hline 40000 & 0.011 & 45.2 & 104.1 & 83.3 & 0.8 \\
\hline
\end{tabular}

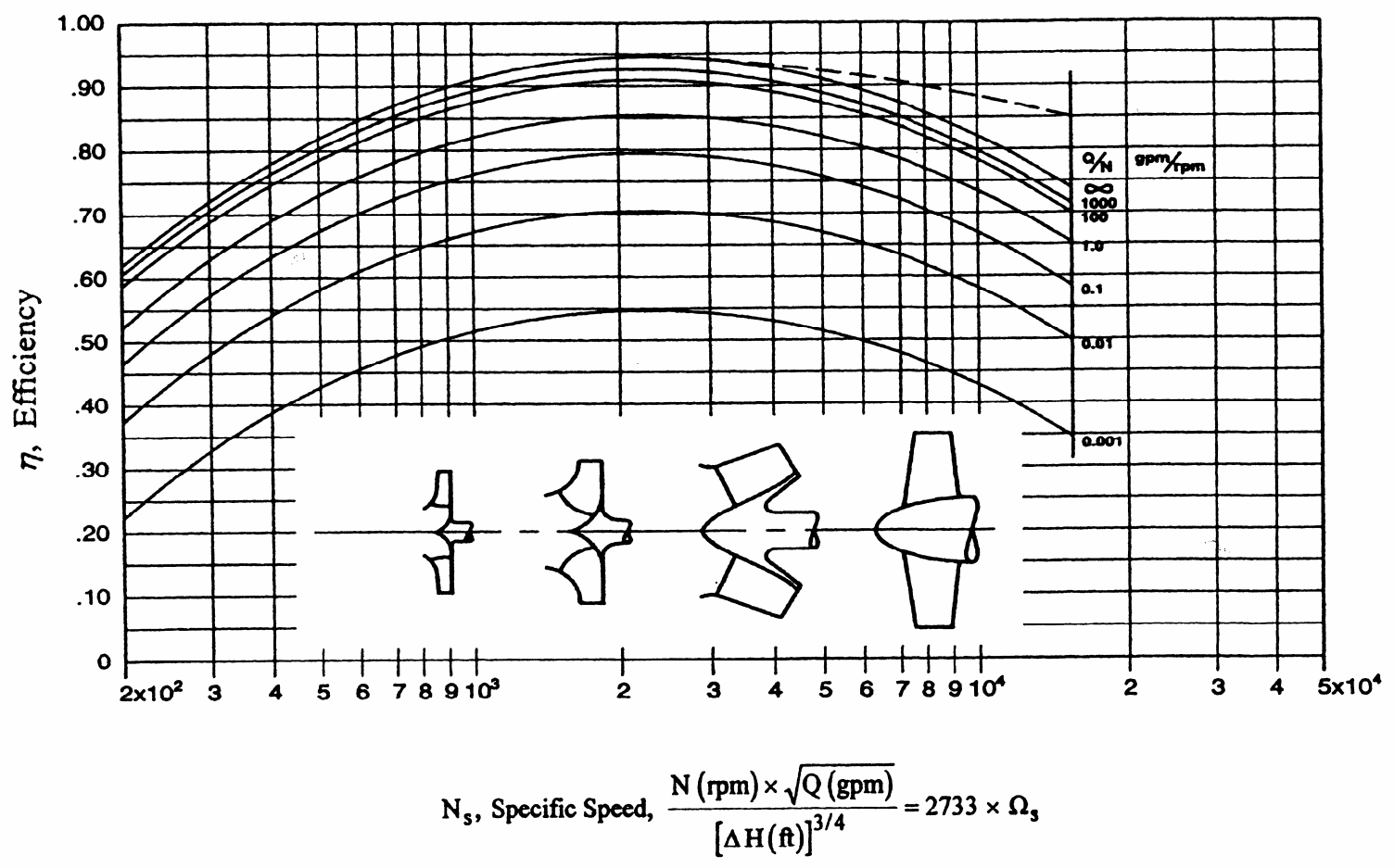

Figure 8: Efficiency $\left(\eta_{\text {overall }}\right)$ of centrifugal pumps versus specific speed, size, and shape (Anderson 1980). 


\section{Summary of Findings}

A single stage configuration is the best suited to meet the design requirements. The blower type is centrifugal, the specific type of centrifugal blower has to be determined (Bleire 1997). An rpm range of 20,000-30,000 with an impeller size of 1 - 2", will result in the best efficiency. This is as far as one can go theoretically. These points will have to be verified through CFD simulations and experimental data.

Major conclusions from this section are summarized below:

- Blower configuration: single stage

- Impeller type: Centrifugal blower

- Specific speed value to ensure highest efficiency approximately 1500 - 2000 (US units)

- $\quad$ rpm value in the range of 20,000 - 40,000 (lower end preferred)

- Impeller size expected $\left(D_{2}\right)$ about $2 "(.05 \mathrm{~m})$ in diameter

- Exit velocity head of considerable magnitude, especially at higher rpm values

- Velocity head can be converted to static pressure using a diffuser

Further, since the blower type has been determined to be centrifugal, referring to the pressure value of $1000 \mathrm{~Pa}$ as 'low' is seems appropriate. Next step (Figure 1) is to determine a base blower design that can be simulated numerically using computational fluid dynamics software (FLUENT 6.1.22, FLUENT, Inc. Lebanon, NH). The subsequent sections cover this. 


\section{BLOWER BASE-DESIGN SELECTION}

According to a European Commission report (2001), pumps are the single largest user of electricity in industries of the European Union. This observation would probably hold for other industrialized countries as well. It is understandable that considerable research has been performed to improve the design and performance of pumps. Indeed, during the last 5 years, the world has generated an average of 400 new patents per year in relation to the design of blowers alone (Mann 2004). It is natural to assume that the blower design has achieved considerable maturity, and the available designs can be modified to meet future design requirements.

Ideally, the selection of a blower design is a simple task. Once a blower configuration and type is finalized, based on the system requirements (described earlier in the section on blower type selection), one consults the promotional literature of a blower manufacturer and chooses from the range of blower designs and sizes. Referring to a catalogue for the present case was not possible as it appears that most of the available literature pertains to large flow rate, high pressure, and high power consuming waterpumps.

In the absence of relevant information and off the shelf designs for low-flow rate, and low pressure rise blowers, one is left with two possible design approaches. The first is to start from ground zero and use the basic design principles, rules of thumb, and computational fluid dynamics (CFD) develop the entire geometry - the blade shape, the inlet and exit angles, leading edge shape, etc., for the centrifugal blower. This is the direct design approach mentioned earlier. It is an arduous task. In the literature this approach is used, albeit very rarely, when dealing with specific diverse pump performance problems and extremely different design conditions. Examples include pumps for fuel handling, heart pumps, pharmaceuticals, and such specialty cases. It is 
always easier, and practical, to modify an existing high efficiency design than to recreate a new blower design. This is covered next.

The other possible alternative is to build on a blower design already available. Here one starts off with a general survey of the available impeller technology, to select a base blower design. To confirm that the blower is well designed, experiments are carried out and design features compared with the recommendations given in the literature. Once a design is fixed, the internal flow-field is studied using CFD. The reasoning being that the development of new and improved centrifugal fans requires the flow-field within the impeller passages to be better conditioned than has been historically possible using empirical overall fan performance test techniques. The objective is to identify, and then eliminate separated and poorly conditioned flow regions. This is essential if the aerodynamic efficiency and pressure development of the blower is to be improved. Besides optimizing the geometry using CFD, fan scaling is used to provide the appropriate size to meet the design goals. The guidelines developed in the preceding sections are used for this.

\section{Non-traditional Scaling}

Conventionally, fan scaling has been oriented towards maintaining the dimensional similarity and it has focused, for the most part, on scaling up rather than scaling down. To maintain dimensional similarity, all dimensions (for example, diameter, width etc.) are scaled by the same ratio. In practice errors do occur, as it is not possible to scale such things as material thickness, or even material roughness. Another area where departures from dimensional similarity occur is the inlet cone clearance on centrifugal fans. Usually, however, in the relative sense these clearances are reduced with increasing size which, more often than not, aids fan performance. Also the roughness of the impeller and case material, over which the air passes, will remain constant as it is scaled up. This leads to what is known as the scale effect where, in general, a larger fan will be more efficient than a smaller one. This happens because the ratio of the static boundary layer to actual 
flow area decreases with increasing size (Halstead 2004). A manufacturer prefers scaling up because of the scale effect and as it allows them to perform model testing on smaller blowers. Increases of $2-3 \%$ are achievable with scaling factors of 2.5 to 3 ; indeed, scaling down is frowned upon in some cases (Halstead 2004).

To overcome these limitations, incorporating CFD into the design process is useful. Using CFD, non-traditional scaling can be used and the ill effects of scaling down can be characterized. Non-traditional scaling refers to the ability to scale different blower dimensions independently of each other, and to visualize their effects. This is a powerful tool, made possible by CFD. It allows better customization of the blower to meet the design requirements. Further, performance reduction, as the blower is scaled down, can be observed and this aids in the design process.

\section{Design Approach}

Preceding discussion points towards the modification and scaling of an existing efficient design as the logical design approach. The approach is more representative of reverse engineering. Customized development of a blower from ground zero to meet the design requirements may produce the best design, but the experience required and the investment needed, in terms of time and money, make it a non-viable option, and even then performance improvement is not guaranteed. Going about the blower design process from ground zero can be a future recommendation, if acceptable performance levels are not achieved. Even though scaling-down a blower might compromise a few efficiency points, it is the best suited as it recognizes the fact that the pump industry has matured in its design. 


\section{Probable Base Blower Designs}

The Aerosol Technology Laboratory (ATL) of the Texas A\&M University System had been experimenting with certain commercially available blowers for another research project. These blowers are being used on aerosol sampling systems designed for high flow rates $(\sim 1000 \mathrm{~L} / \mathrm{min})$, and high pressure $\operatorname{rise}\left(\sim 30^{\prime \prime} \mathrm{WC}\right.$ or $\left.7500 \mathrm{~Pa}\right)$. The power consumption was about $600 \mathrm{~W}$ (Moncla 2004). Even though these values are an order of magnitude higher then the design requirements the blowers can be used as a probable initial design and their performance measured. The impellers used in these blowers are centrifugal turbo blowers (Bleier 1997) with nine $\left(Z_{9}\right)$ backward curved (BC) blades.

Another appropriate place to look for air-handling centrifugal blowers is the hand-held vacuum systems (HVS) available in the market. It can be reasoned, that the fans used in these hand-held systems should be a product of good engineering design. Firstly, these portable vacuum systems are battery operated, therefore the fans used in them need to be as efficient as possible to maximize performance and endurance. An improvised homemade experiment, with one of these blowers, showed that the system generate a suction pressure of about $8-10 " \mathrm{WC}(\sim 2000 \mathrm{~Pa})$. Even though the overall efficiency of these systems might be low as they run on small, cheap, off-the-shelf direct current (DC) motors, the blower design has to be good for it to generate $8-10^{\prime \prime} \mathrm{WC}(\sim 2000 \mathrm{~Pa})$ of pressure and a reasonable flow rate. These also have nine blades. The two blowers are shown below (Figures 9 and 10). Design differences between the two blowers are apparent. 


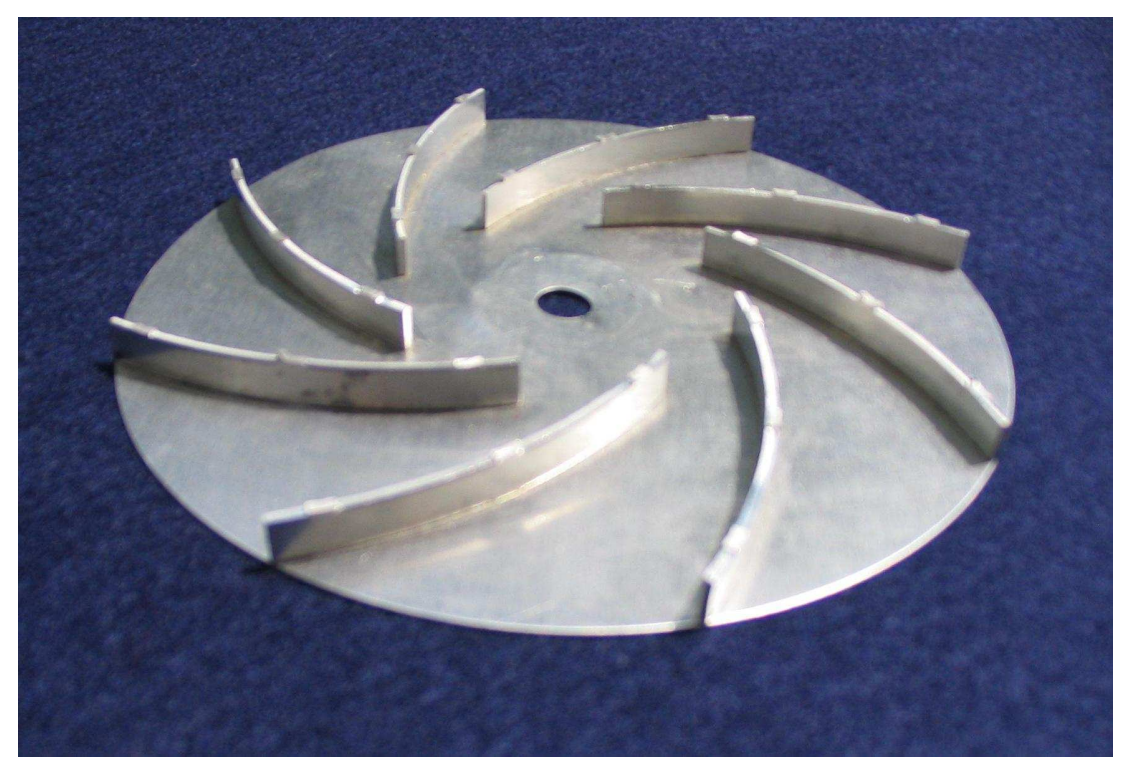

Figure 9: Turbo blowers with backward curved blades (shroud removed).

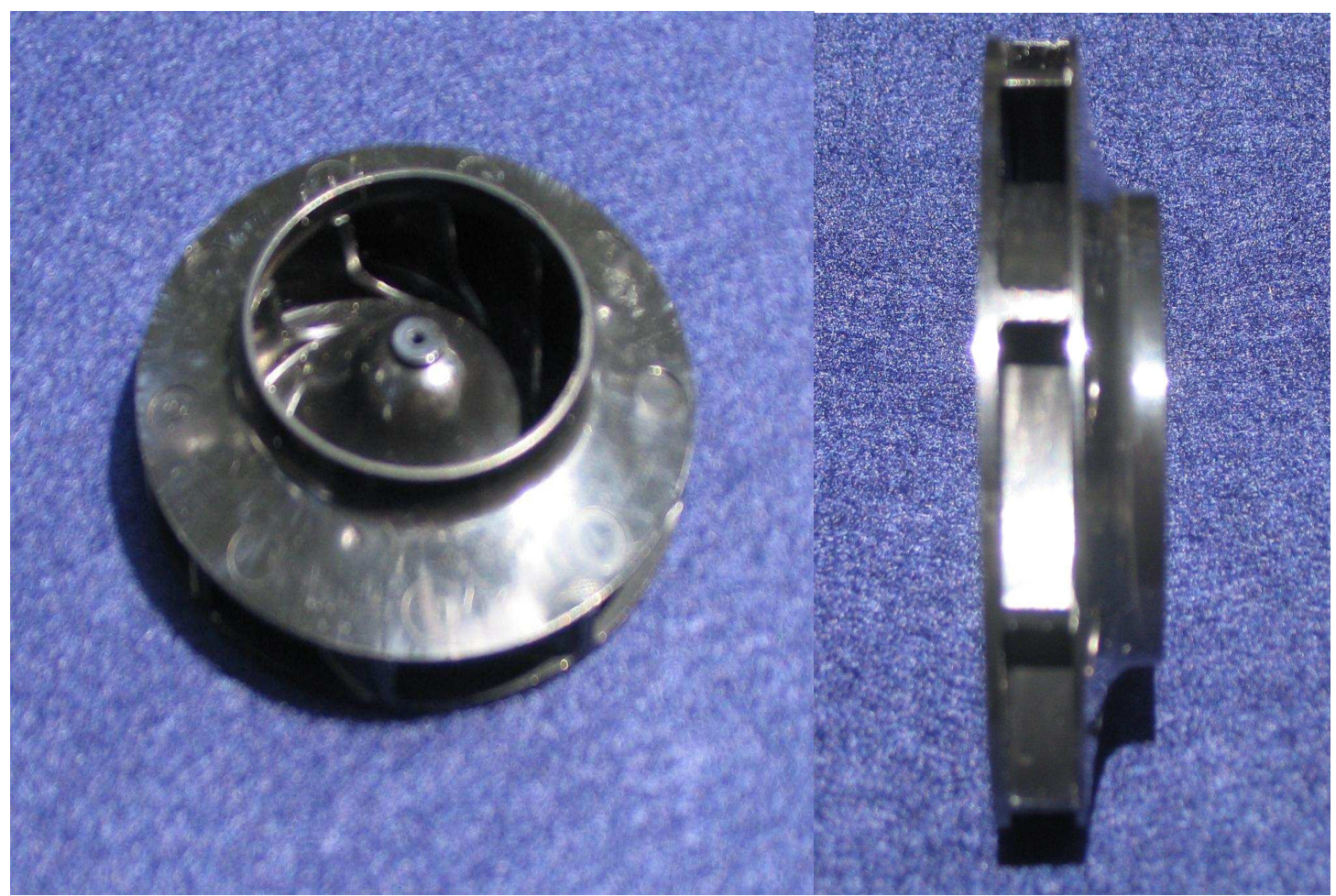

(a)

(b)

Figure 10: Hand-held vacuum system centrifugal blower (a) Top-view (b) Side-view showing the blades. 


\section{Selection of the Hand-held Vacuum System (HVS) Blower: Justification}

The two blowers mentioned in the last section were:

- Turbo blowers (Bleier1997), with slightly backward-curved (BC) blades, being already tested by the lab (Figure 9)

- Centrifugal blowers, with backward-curved (BC) blades, (Bleier1997) adopted from hand-held vacuum devices (Figure 10)

Comparison between the two designs suggests that not much engineering was incorporated into the design of turbo-blowers. Their blades are narrow, circular arcs of uniform height, and are riveted to the back plate and shroud. In contrast, the HVS blower with $\mathrm{BC}$ blades has blades that vary in height from the leading edge to the impeller tip. Another important factor favoring HVS blowers over turbo-blowers was the shape of the leading edge. The design of the impeller leading edge is particularly important (Japikse 1997). Figure 11 shows a series of different inlet configurations. Turbo-blower, shown on the left (a), is a simple two-dimensional blade following a region of moderately sharp shroud line curvature. This is a poor design feature and can lead to separation and backflow out of the impeller eye (Figure 12). The centrifugal blower with BC blade has a moderately three-dimensional shaped impeller (c in Figure 11) inlet. This allows for better incidence control (Japikse 1997).

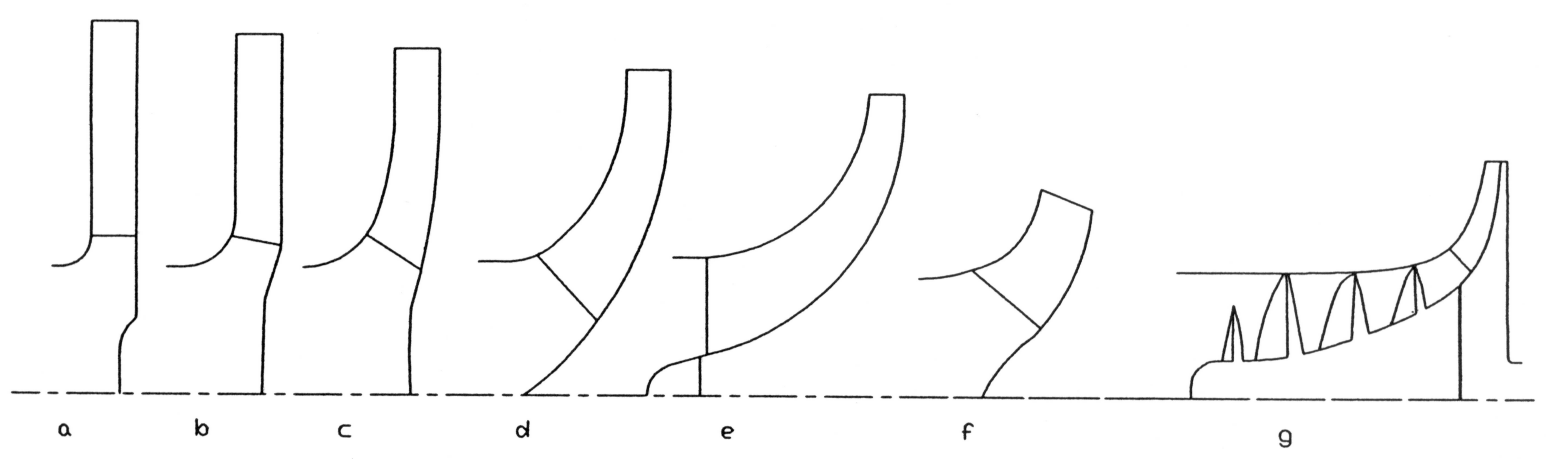

Figure 11: Variety of common pump impeller meridional views from low to high specific speeds $(\mathrm{a}-\mathrm{f})$ and with inducer $(\mathrm{g})$. 
Further, according to their blade shapes, centrifugal fans can be subdivided into six categories (Bleire 1997): Airfoil (AF), backward-curved (BC), backward-inclined (BI), radial-tip (RT), forward-curved (FC), and radial blade (RB). Figure 13 shows these six commonly used blade shapes. Each of them has its advantages and disadvantages. Accordingly, each is well suited for certain applications. Figure 13 also shows the approximate maximum efficiencies that usually can be attained with these blade shapes (these efficiency values are for large flow rate fans used in the industry, but the trend should remain the same for smaller sized blowers). Just on the basis of the maximum efficiency attainable, the hand-held vacuum blowers are a better choice.
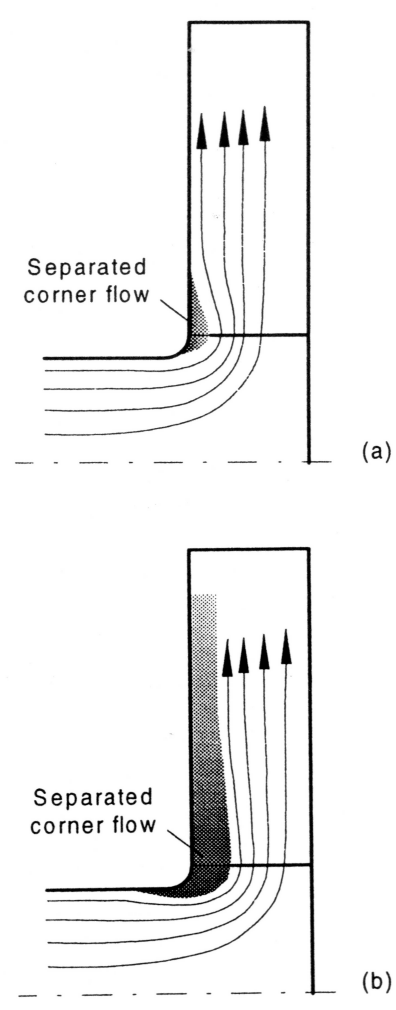

Figure 12: Impeller with strong inlet curvature (a) Separation with reattachment (b) Complete separation without reattachment. 


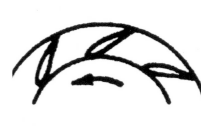

AF

$92 \%$

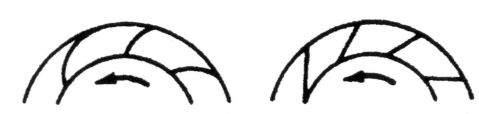

$\mathrm{BC}$

BI

$78 \%$

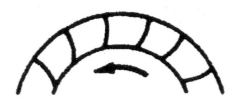

RT

$70 \%$

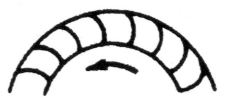

FC

$65 \%$

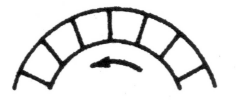

RB

$60 \%$

Figure 13: Common centrifugal fan blade shapes.

It can be argued, based on the efficiency values (Figure 13), that AF centrifugal blowers will be a better choice. Still, it was decided to go ahead with BC blades as these are easier to manufacture. Besides, in centrifugal fans, the improvement due to AF blades is not as pronounced (Bleier 1997) as the airfoil lift contributes only a small portion to the pressure produced, and most of it is generated by the action of the centrifugal force.

In view of these arguments, the HVS blower with backward curved blades and inclined leading edge (referred as 'blower' through the remaining text) was chosen as the design base. The performance level of the blower will have to be determined, both numerically and experimentally, to verify that the design is acceptable. This is done in the following sections. Other dimensions, describing the blower geometry, are presented in the section on CFD (Figure 14). 


\section{COMPUTATIONAL FLUID DYNAMICS (CFD)}

\section{Background}

The objective here is to design, develop and test a centrifugal blower to meet the specific flow rate and pressure rise requirements, and have acceptable efficiency levels. According to Turton (1984) a successful impeller design should, “...produce a specific pressure rise and flow rate within acceptable limits, at an acceptable rotational speed, and require minimum power from its drive; also, it must exhibit stable characteristics over the operating range required. The impeller must be as small as possible, the power absorbed must be non-overloading over the flow range and the noise and vibration must be within specified limits. The design must always be economical, give good quality assurance, and be easily maintained..."

Designing a blower that meets all the above mentioned parameters is a daunting task and a challenging research area. To design an impeller, it is necessary to predict its performance curves. Performance prediction design process has classically evolved from a fully empirical process in the 1950 's, through to a one dimensional analysis in the 1960 's. It continued with the axis-symmetric through flow calculations in the 1970's, with full three dimensional calculation of the blade to blade flow field becoming routine during the 1980's (Tallgren 2004). Early 1990's saw the development of Quasi Threedimensional Analysis (Q3D) technique as a blade design tool (Katsanis 1991).

Flow patterns in a centrifugal pump are three-dimensional, unsteady, and characterized by re-circulation, cavitation (for water pumps), and pressure pulsations. The early methods cannot handle the complexity of such flow-phenomenon. Hence, the later part of 1990 saw increase in computational capability enable the use of three-dimensional computational techniques during the design process itself (Tallgren 2004), instead of just the post design analysis of blade performance. 
Ever increasing computational power and robust turbulence models enabled CFD to become a powerful tool. Solving three dimensional Reynolds-averaged-Navier-Stokes equations has become routine. CFD has been used to predict recirculation, and the results compare favorably with experimental data (Graf 1993). Gülish et al. (1997) predicted the entire performance curve of head within about two percent, and the power curve with slightly less accuracy. Lakshminarayana (1990) assesses the use of CFD techniques in the analysis and design of turbo-machinery. Further, CFD is being used in the design of unconventional pump applications, like blood pumping devices (Koh 1999). All this highlights the fact that CFD allows a systematic, robust, and economically viable approach to pump design.

\section{CFD Approach}

A common factor applicable to all CFD studies of turbo-machinery is that the CFD does not eliminate the need for experimental validation; it does however compliment it. This is achieved by using measurements, which are relatively quick and easy to make, to verify that a CFD model is producing results that are predicting the overall performance correctly. The CFD model can then be assumed to model the flow adequately and as such used to provide information on the flow-field. This is exactly the approach taken here.

Once we have confidence that CFD is predicting the measured performance of a blower reasonably accurately, a study of the CFD results facilitates insights into aspects of the flow field that are limiting fan performance. This in turn offers the possibility of making changes to the CFD model in an attempt to improve the predicted performance (nontraditional scaling). Once predicted performance has been improved, possibly after many different design iterations, the optimized geometry is fabricated, and overall fan performance again verified empirically.

The above two paragraphs describe the essence of the remaining thesis. The blower model will be built in a Computer Aided Design (CAD) package, Inventor 8 (Autodesk 
Inc., San Rafael, CA). The performance of the blower will be predicted using a commercial CFD package - FLUNET 6.1.22 (FLUENT Inc, Lebanon, NH). Predicted blower performance will be validated against experimental results; for this an experimental test rig will be designed (explained in the subsequent sections). Once CFD predictions are in general agreement with the experimental data, changes to the blower model will be made; these include changing the impeller size, the blade height, etc. to achieve improved predicted performance at the design point. Then the optimized fan geometry can be fabricated, and overall fan performance again verified experimentally. An additional advantage of CFD, mentioned earlier, is the flow visualization that is possible. This helps understand how different flow features such as separated flow regions or secondary flow vortices, are developing through the fan. To gather sufficient data to do these using empirical techniques is simply impractical in practice.

\section{FLUENT 6.1.22 - Introduction}

FLUENT 6.1.22 is a state-of-the-art Computational Fluid Dynamics (CFD) program for modeling fluid flow and heat transfer in complex geometries. FLUENT uses the finite volume (FV) formulation to discretize the governing equations of mass conservation, force balance, energy conservation (if needed), and species conservation (for multiphase modeling) over complex problem domains. To support these functions FLUENT incorporates an extensive range of turbulence models, buoyancy models, species transport models, etc. (FLUENT User's Guide 2004).

Any modeling approach using CFD has the following sequence:

- Physical model is either created in the geometry setup module or is imported from other standard CAD design packages

- The physical model is meshed. This is a critical step as the mesh used should be able to capture the flow physics

- A physical model has to be chosen depending on the type of flow being modeled, for example, inviscid, laminar, or turbulent 
- Various boundary and initial conditions are specified

- The discretized representation of the governing equations over the flow domain is solved

- Post processing and analysis of the flow solution is done

In FLUENT these functions are split between GAMBIT and the FLUENT solver. GAMBIT is used to define the geometry and create the mesh. GAMBIT offers great flexibility in meshing the problem domain and allows for triangular, quadrilateral, tetrahedral, or hexahedral cells. The grid can be structured or unstructured.

The FLUENT solver defines the physical model i.e. laminar, turbulent, combustion, multiphase, etc., specifies the various boundary conditions, solves the finite volume equations over the discretized domain, and post processes the results. An inbuilt postprocessor is a useful feature available in FLUENT.

\section{Blower Geometry for CFD Simulations}

The hand-held vacuum system (HVS) blower geometry was created in Inventor 8. Most of the dimensions could be easily measured except for the inlet blade angle, the exit blade angle, and dimensions of the blower leading edge. These values were approximated. The blower geometry, with the characteristic dimensions, is shown in Figure 14.

The main characteristic dimensions of the blower are:

- Impeller eye diameter $\left(d_{e}\right)=1.58 "(40 \mathrm{~mm})$

- Impeller outlet diameter at blade trailing edge $\left(D_{2}\right)=2.7 "(68.6 \mathrm{~mm})$

- Impeller blade height at exit $\left(b_{2}\right)=0.29^{\prime \prime}(7.4 \mathrm{~mm})$

- Blade angle at leading edge $\left(\beta_{1}\right)=13.07^{\circ}$ and $23.05^{\circ}$

- Blade angle at trailing edge $\left(\beta_{2}\right)=28.92^{\circ}$ and $31.92^{\circ}$ 


\section{Hand-Held Vacuum System Blower}

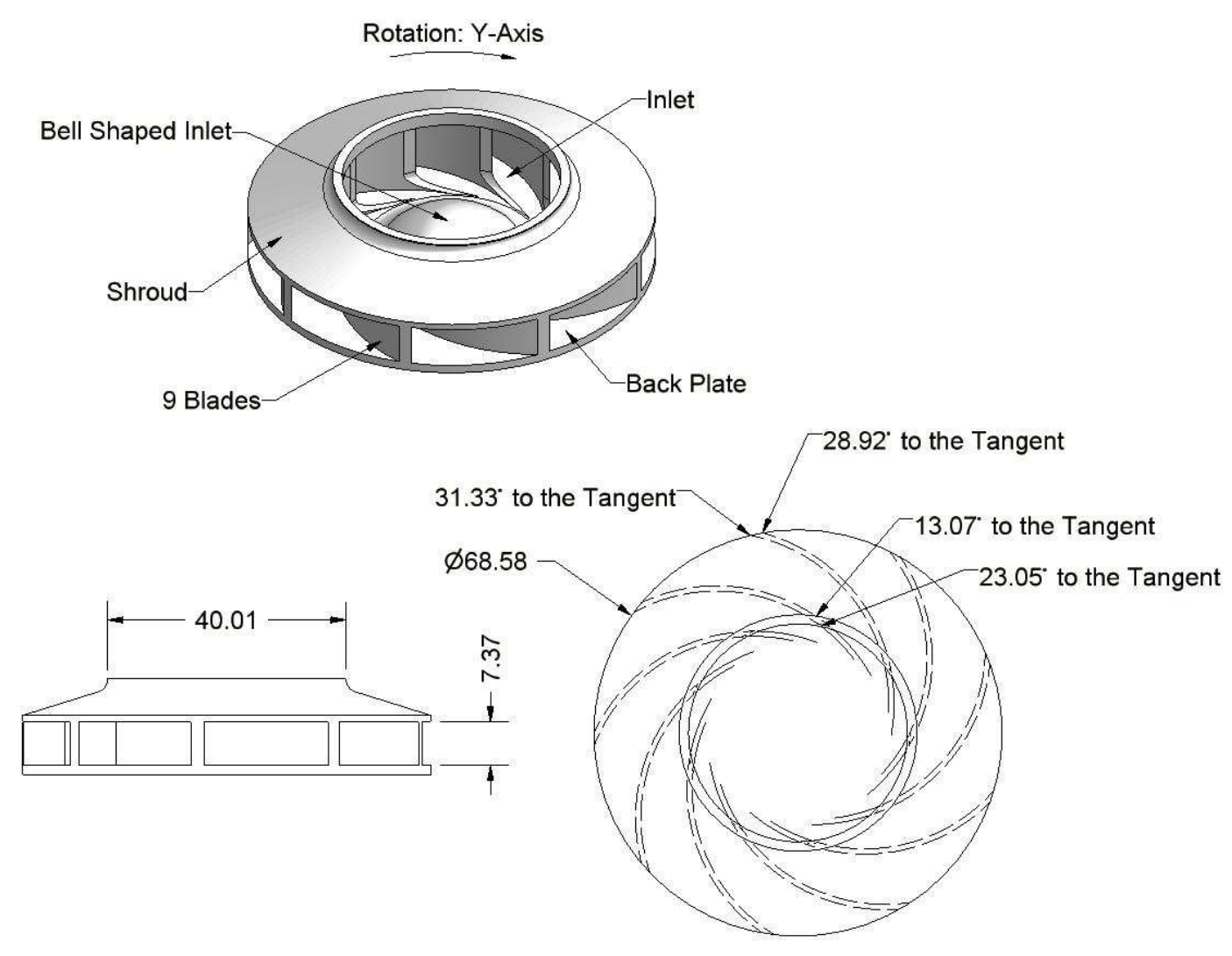

Figure 14: Base blower dimensions (mm). 


\section{GAMBIT Model}

The geometry of the blower is rotationally symmetric. This implies that one can divide the blower geometry into sections since that the flow physics in each section is the same, except for the rotation about the axial direction. This reduces the computational effort as it requires only one passage of the impeller to be simulated. The approach of simulating a single blower passage is common in literature (Han et al. 2002). The only probable drawback might be slightly lower exit velocities as the blade thickness is not incorporated in the model

In the present case, the blower has 9 blades, implying that a computational domain bounded by the blade profiles and with an included angle of $40^{\circ}$ will be adequate to capture the flow-field. The GAMBIT model is shown in Figure 15. The volume was closed by extending the blade walls to the origin of the rotation axis. To do this, periodic boundary conditions: periodic boundary $-\mathrm{a}$, and periodic boundary $-\mathrm{b}$ (Figure 15), were created. Periodic boundary conditions are used when the physical geometry of interest and the expected pattern of the flow/thermal solution have a periodically repeating nature (FLUENT User's Guide 2004). 


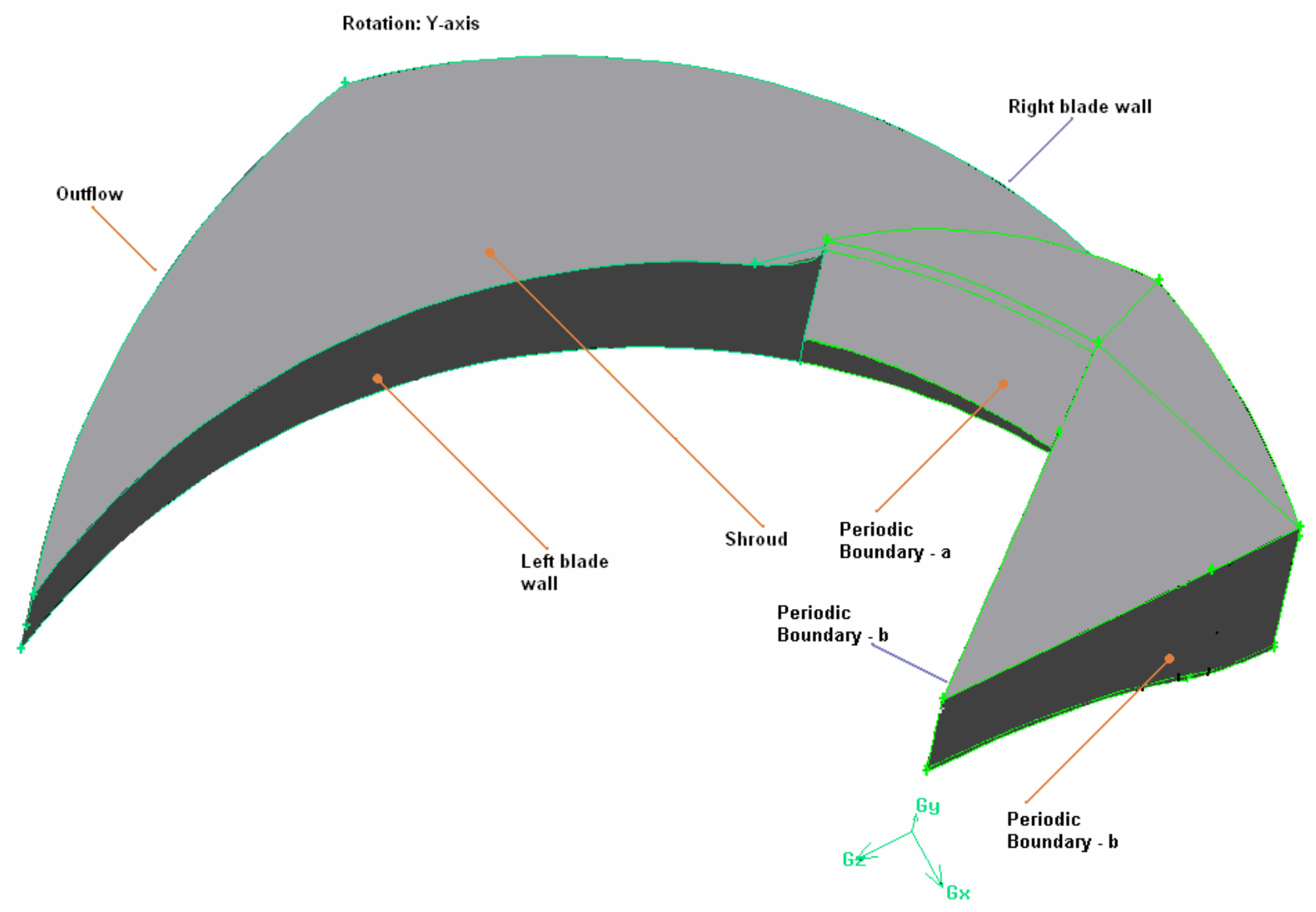

Figure 15: CFD model created in GAMBIT.

\section{GAMBIT Meshing}

An acceptable mesh is crucial in reaching a converged solution that captures all the key parameters of the flow. On the other hand, too many nodes in the system may increase the computational resources and time without providing additional resolution. These two requirements go against one another. Generating the mesh is a critical step in CFD analysis. A mesh captures the flow physics and requires considerable experience with CFD and an ability to visualize the flow. General guidelines do exist for creating acceptable meshes (ERCOFTAC Best Practice Guidelines 2000, GAMBIT 2.1 User's Guide 2004). 
For the present case, hexahedral mesh elements were used all through the CFD model and hence, the grid was the structured type. Cooper meshing scheme was used in which a face is meshed first, called the source face. The source face-mesh is translated through the volume. The mesh is shown in Figures 16 (a) and (b). The face representing the mass inlet was meshed using tri-primitive mesh scheme with quadrilateral elements. This was done due to the shape of the inlet faces. The impeller shroud face was meshed using quadrilateral elements and submap meshing scheme (GAMBIT User's Guide 2004).

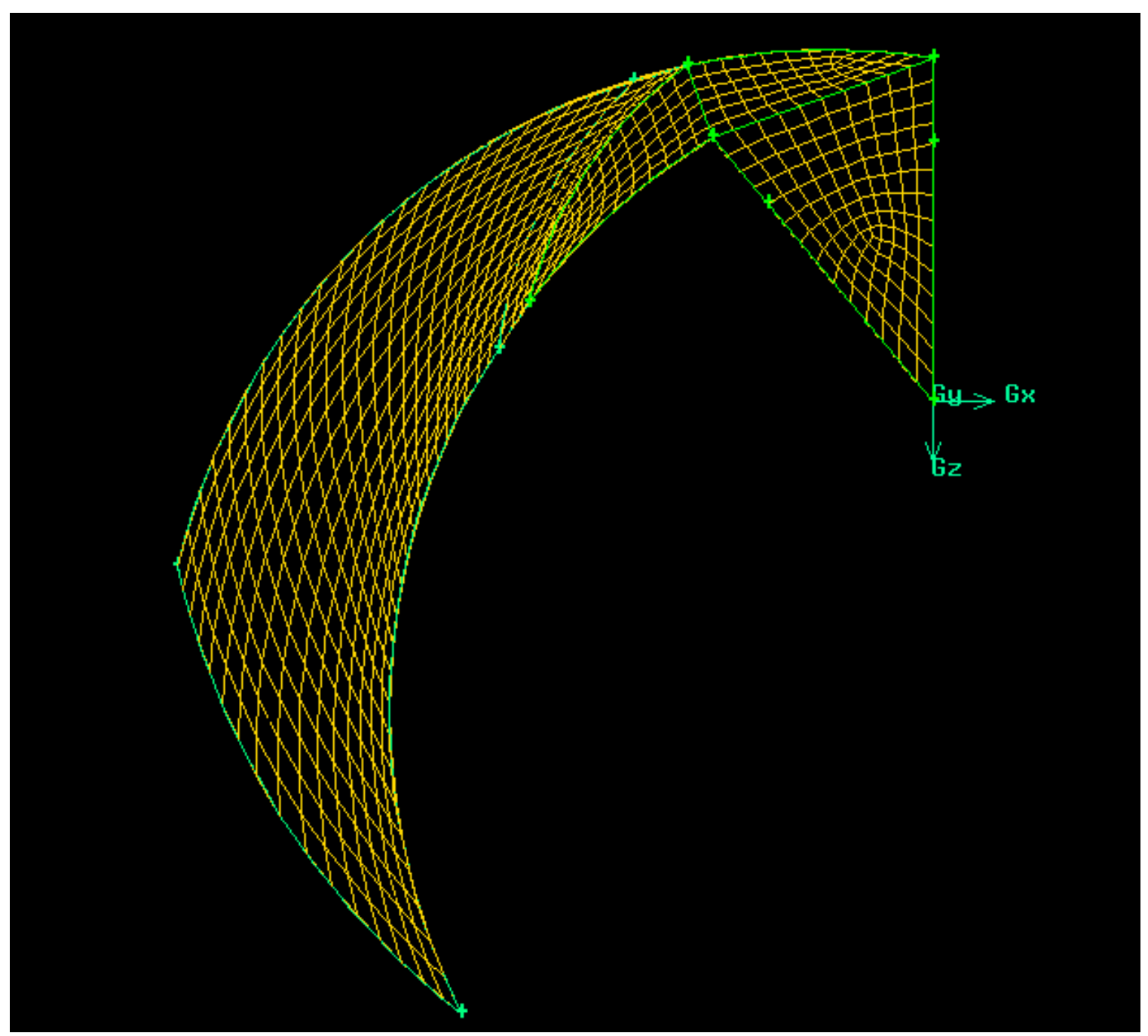

Figure 16: CFD model mesh: (a) Top-view 


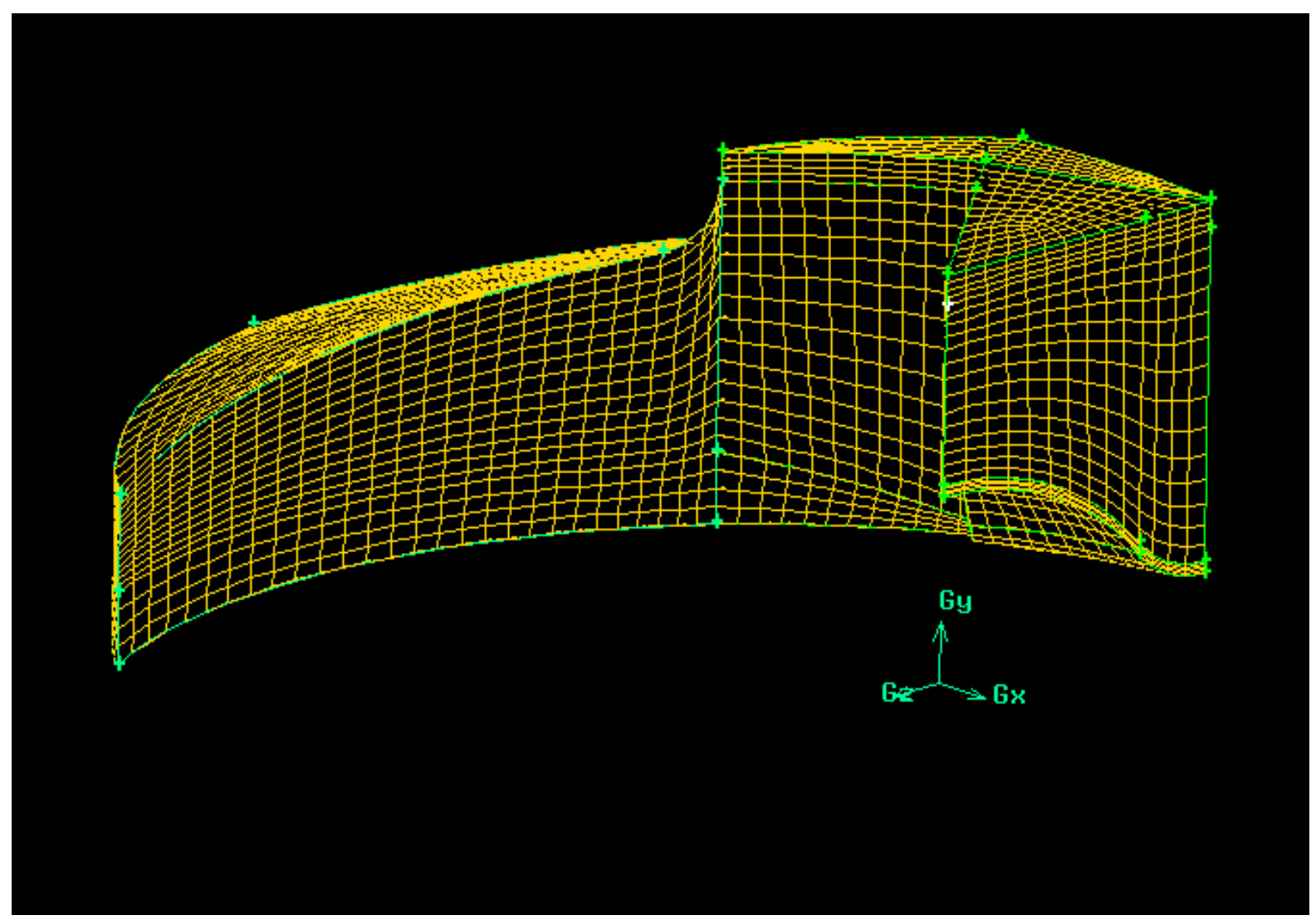

Figure 16: continued: (b) Side-view

Mesh-quality was verified using the equiangle skew algorithm (GAMBIT User's Guide 2004). Each element has a value of skewness between 0 and 1, where 0 represents an ideal element. Values less than 0.98 are acceptable. For the present case, because of the complex shape of the flow-domain, majority of the elements have a skewness value less than 0.8. This information is represented in a histogram, with the skewness values on the horizontal axis. Figure 17 shows the skewness distribution for the blower mesh. The total number of grids cell was 14000 . 


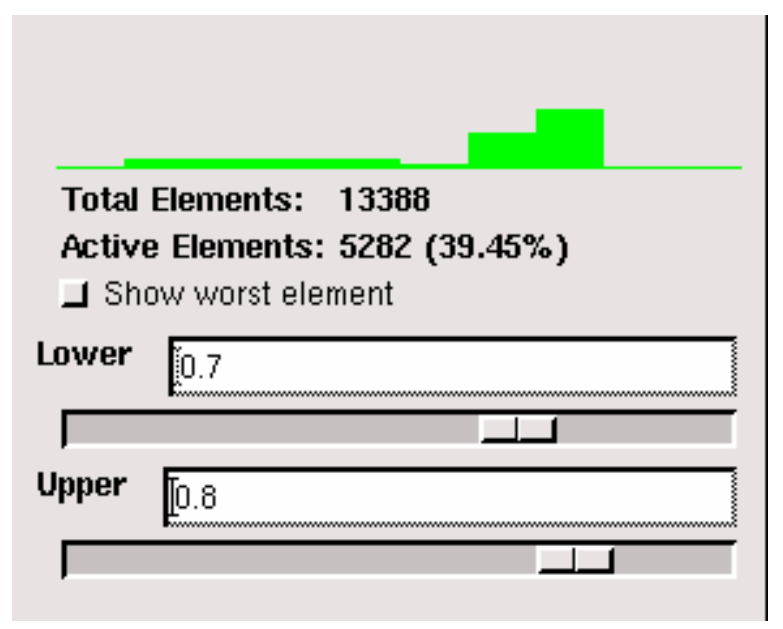

Figure 17: Mesh quality: Skewness value for mesh cells.

\section{FLUENT Model}

Fluid flow in a rotating machine is characterized by a rotational (or machine) Reynolds number, Re. It is defined based on the rotational speed $(\Omega, \operatorname{rad} / \mathrm{s})$, the impeller exit $\operatorname{radius}\left(r_{2}, \mathrm{~m}\right)$, fluid density $\left(\rho, \mathrm{kg} / \mathrm{m}^{3}\right)$, and the dynamic viscosity $(\mu, \mathrm{kg} / \mathrm{m} . \mathrm{s})$. For the impeller considered first in this study, the machine Reynolds number is of the order of $10^{5}$ (Equation 22).

$$
\begin{aligned}
& \rho=1.225 \mathrm{~kg} / \mathrm{m}^{3}(\text { FLUENT }) \\
& \Omega=2094 \mathrm{rad} / \mathrm{s}(20,000 \mathrm{rpm}) \\
& R_{2}=0.034 \mathrm{~m} \\
& \mu=1.7894 \times 10^{-5} \mathrm{~kg} / \mathrm{m} . \mathrm{s} \\
& \operatorname{Re}=\frac{\rho . \Omega \cdot R_{2}^{2}}{\mu}=\frac{\left(1.225 \mathrm{~kg} / \mathrm{m}^{3}\right) \cdot(2094 \mathrm{rad} / \mathrm{s}) \cdot(0.034 \mathrm{~m})^{2}}{\left(1.7894 \times 10^{-5} \mathrm{~kg} / \mathrm{m} \cdot \mathrm{s}\right)}=1.66 \times 10^{5}
\end{aligned}
$$




\section{Turbulence Model}

The flow field, based on the machine Reynolds number, is definitely turbulent. One has to choose a turbulence model which encapsulates the physics of the flow and produces the most realistic results. The simplest "complete models" of turbulence are two-equation models in which the solution of two separate transport equations allows the turbulent velocity and length scales to be independently determined. The standard $\kappa-\varepsilon$ (Launder et al. 1972) model falls within this category and has been the most widely used for engineering calculations.

The $\kappa-\varepsilon$ model is acceptably accurate for simple flows, but it can be quite inaccurate for complex flows (Pope 2001). Flow within an impeller rotating at 20,000 rpm is complex. The difficulties associated with solving swirling and rotating flows are the result of the high degree of coupling between the momentum equations, which is introduced when the influence of the rotational terms is large. A high level of rotation introduces a large radial pressure gradient that drives the flow in the axial and radial directions. This, in turn, determines the distribution of the swirl or rotation in the field. To account for these factors, improvements have been made to the $\kappa-\varepsilon$ model. One such variant, used here, is the Renormalization Group (RNG) $\kappa-\varepsilon$ model (Yakhot 1986). FLUENT recommends using RNG $\kappa-\varepsilon$ model for highly swirling flows and provides an option to account for the effects of swirl or rotation by modifying the turbulent viscosity appropriately. For the transport equations the reader is referred to the FLUENT User's Guide (2004) and Pope (2001).

\section{Inlet and Outlet Conditions}

At the inlet boundary, a uniform axial velocity (along the $Y$ axis) is imposed based on the specified mass flow rate. For this, the inlet area is estimated and the inlet velocity magnitude calculated. For the turbulence model, due to a lack of turbulence data from the 
experiment, a turbulence intensity of $2.5 \%$ and a reference length equal to $3 \%$ of the inlet diameter is assumed (Thakur et al. 2002). The outlet boundary is specified as massflow with all other variables being extrapolated. Turbulence intensity and length scale are calculated in a manner similar to inlet boundary.

\section{Near-Wall Treatment}

Another important consideration for turbulence modeling is the near-wall modeling. Turbulent flows are significantly affected by the presence of walls (Tennekes et al. 1997). Further, the $\kappa-\varepsilon$ models are primarily valid for turbulent core flows (i.e. the flow in the region far from walls). Consideration therefore needs to be given as to how to make these models suitable for wall-bounded flows. This is done with the help of wall functions (FLUENT User's Guide 2004). FLUENT provides two options, standard wall functions and non-equilibrium wall functions. FLUENT recommends using the non-equilibrium wall functions for complex flows involving separation, reattachment, and impingement where the mean flow and turbulence are subjected to severe pressure gradients and change rapidly. This is due to of its capability to partly account for the effects of pressure gradients and departure from equilibrium.

A cautionary note to be kept in mind regarding the limitation of the wall functions approach. To quote from FLUENT manual, "The standard wall functions give reasonably accurate predictions for the majority of high-Reynolds-number wall-bounded flows. The non-equilibrium wall functions further extend the applicability of the wall function approach by including the effects of pressure gradient and strong non-equilibrium. However, the wall function approach becomes less reliable when the flow conditions depart too much from the ideal conditions underlying the wall functions". Examples include:

- Severe pressure gradients leading to boundary layer separations

- Strong body forces (e.g., flow near rotating disks, buoyancy-driven flows) 
Both these conditions are relevant for the blower and restrict the applicability of wall functions approach. FLUENT recommends using the enhanced wall treatment for such situations. The only drawback here is that enhanced wall treatment formulation is computationally expensive and time consuming. This difference can be understood in terms of the non-dimensional distance $y^{+}=\frac{\rho \cdot u_{\tau} \cdot y_{P}}{\mu}$, to the wall from the first grid cell. Here $\rho$ is the fluid density, $u_{\tau}=\sqrt{\tau_{w} / \rho_{w}}$ is the friction velocity, $y_{P}$ is the distance from point $P$ to the wall, and $\mu$ is the fluid viscosity at point $P$. The wall functions formulation requires a $y^{+} \approx 30$ (ERCOFTAC Best Practice Guidelines 2000, FLUENT User's Guide 2004) while for enhanced wall treatment $y^{+} \approx 1$ (FLUENT User's Guide 2004). This translates into the number of grid cells being a magnitude higher for the enhanced wall treatment approach. Therefore, the approach adopted here is to use the non-equilibrium wall functions formulation initially, and then switch to enhanced wall treatment if performance prediction is not acceptable.

In the next section, a description of the test assembly is given. The results from the simulations and the test runs are given in a subsequent section. 


\section{EXPERIMENTAL TEST RIG}

CFD quickens and adds flexibility to a design process. A common factor applicable to all CFD studies of turbo-machinery is that the CFD does not eliminate the need for experimental validation. CFDs robustness, for turbulence modeling, is still to be proved. Further, Japikse et al. (1997) mention that for small-size pumps, the existing CFD capability does not work well. Hence to gain sufficient confidence in the CFD model, it is necessary to validate the simulation results with experimental data.

The approach is to make simple measurements, like pressure rise, flow rate, etc. and compare them with the simulation results. A schematic of the test-rig, which was setup for the present study, is shown in Figure 18. It includes a venturi (for flow rate measurement), a valve for flow-control, the centrifugal fan to be tested, and a motor drive mechanism. Other components are explained in the following sections. A photograph of the set-up, showing the venturi flow-meter, the gauges for pressure measurement, and the valve, is given below in Figure 19.

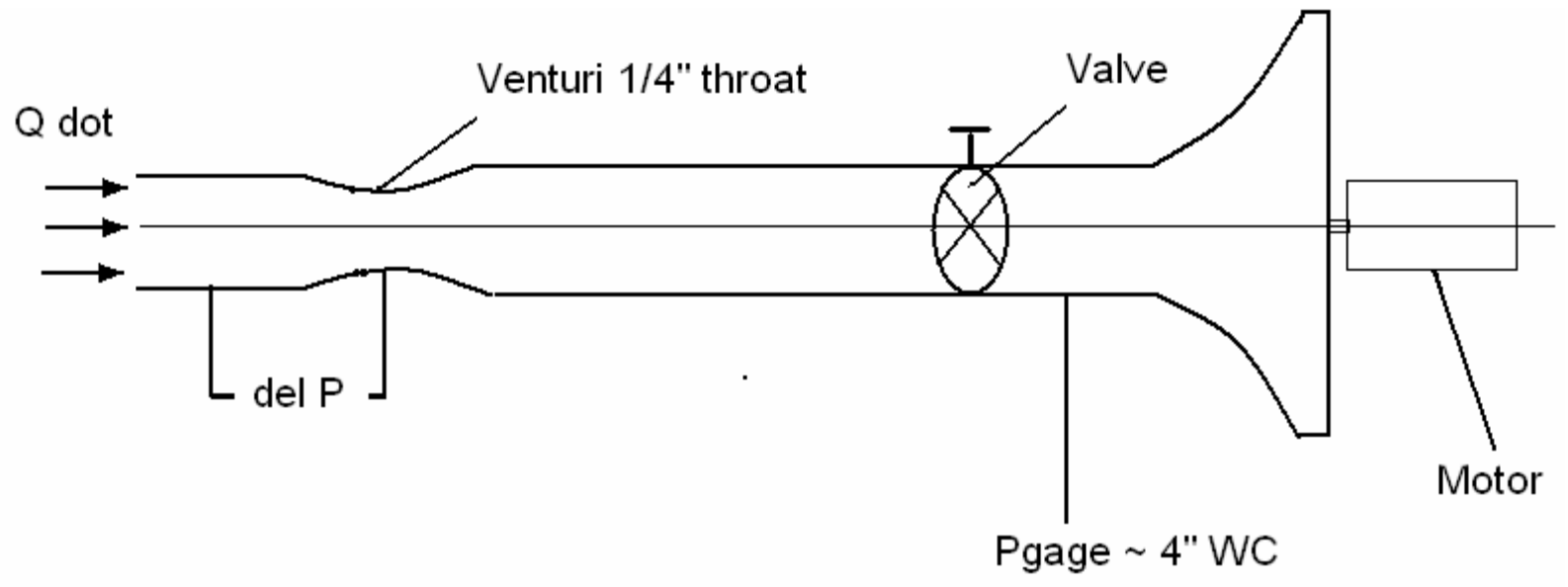

Figure 18: Blower test-rig schematic. 


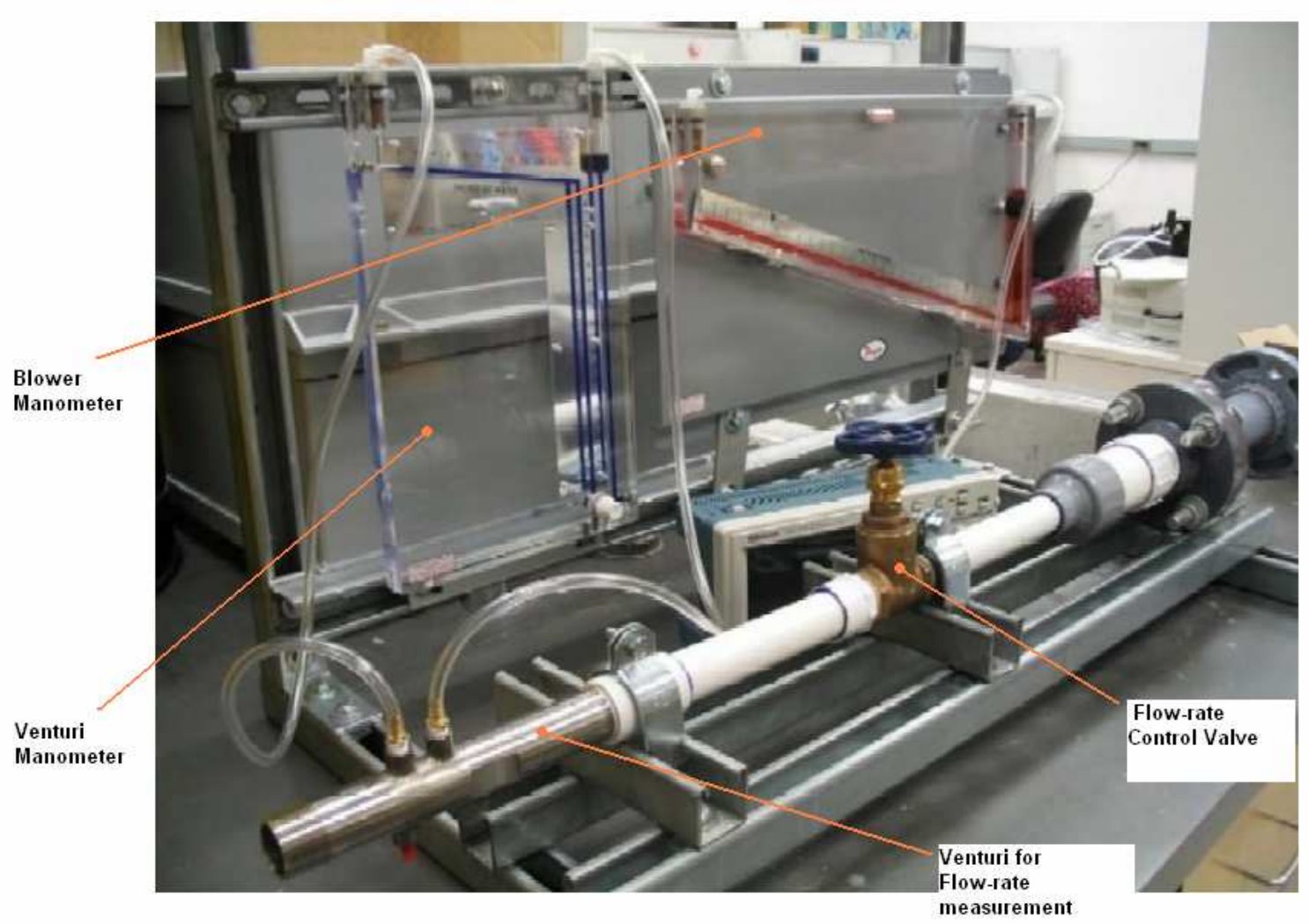

Figure 19: Blower performance measurement test-rig.

\section{Flow Rate Measurement}

A venturi flow meter (FLOW-DYNE Engineering, Inc. Fort Worth, TX) was installed as an inlet section and used to measure the flow of air. The venturi consists of a converging inlet section, a short straight throat section, and a diverging section. A venturi flowmeter was selected because of the high percentage of pressure recovery possible (Figure 20). This was needed because of the low pressure capabilities of the blower involved. Calibration charts were provided by the company (Appendix A). Sample calculation is also shown there. The venturi has a throat diameter $\left(D_{1}\right)$ of $0.25^{\prime \prime}$ and an inlet section diameter of 1.04". 


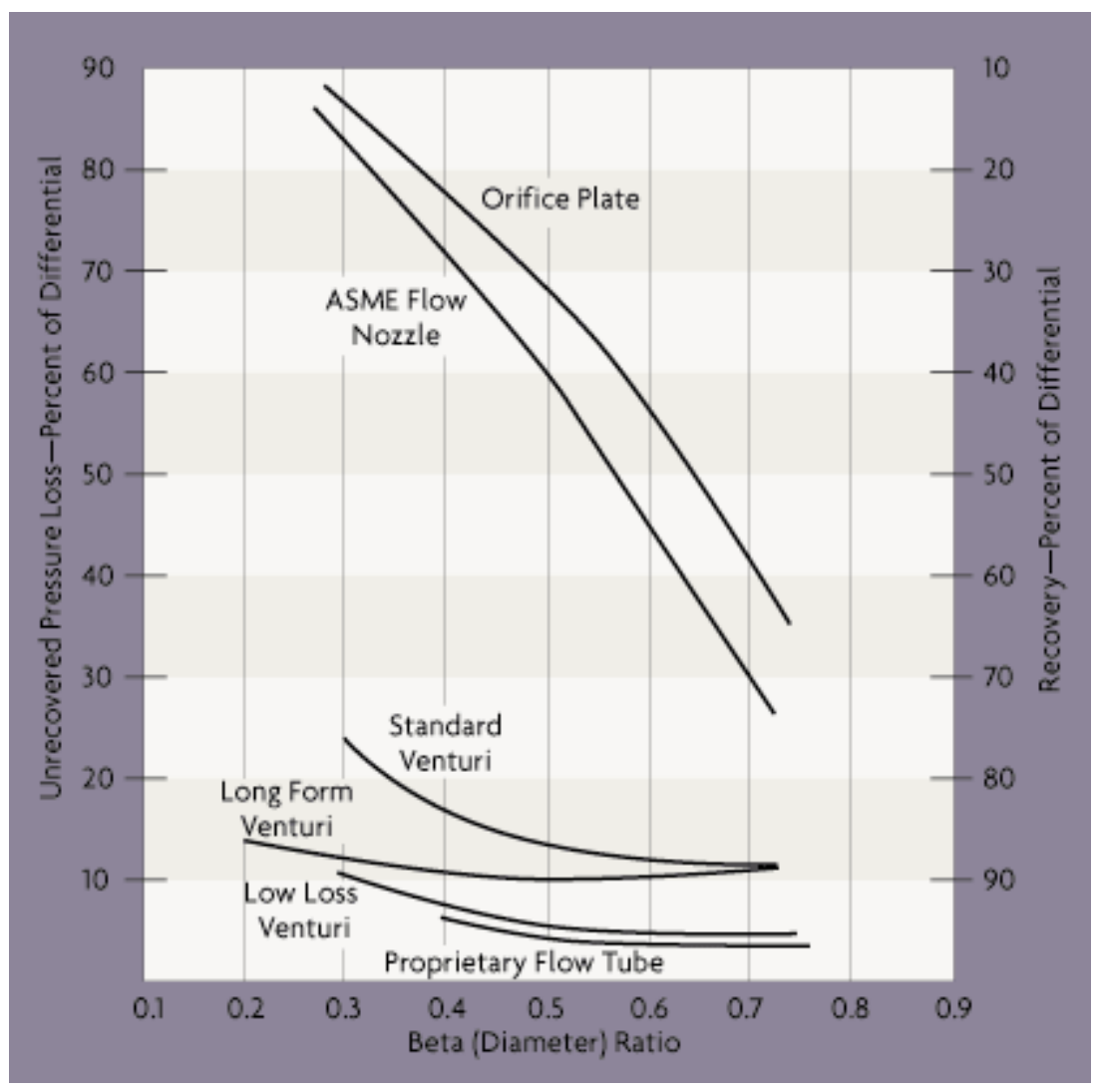

Figure 20: Pressure recovery for different venturi types (FLOW-DYNE Engineering).

\section{Pressure Measurement}

Pressure measurement was done using standard manometers available through Dwyer Instruments (Dwyer Instruments, Inc. Michigan City, IN). The accuracy and the range of the manometers were selected based on the design requirements. Pressure readings were needed at two points: (i) Differential pressure across the venturi inlet and the throat. Expected pressure differential was $15 " \mathrm{WC}(3700 \mathrm{~Pa})$, this covers a volume flow rate of approximately $200 \mathrm{~L} / \mathrm{min}$ based on the venturi calibration curves (Appendix A). (ii) Static pressure reading is needed at the blower inlet region. The manometer here measures the suction pressure created by the blower. The expected negative pressure range was $4-6$ " WC $(1000-1500 \mathrm{~Pa})$. 


\section{Blower Drive}

FLUENT simulations need to be validated against experimental results. The approach is to:

- Simulate the hand-held vacuum system blower

- Run tests with the blower

- If agreement is acceptable, modify the blower design to optimize performance at the design point

- Modify the test-rig for the final design and test again

At this point, the torque requirements from the blower are not known, so an appropriate motor cannot be selected. For testing the hand-held vacuum system blower, it was decided to use the original motor supplied with the vacuum system. Later, once the torque requirements are known from FLUENT an appropriate motor will be chosen.

\section{RPM Measurement}

A fiber-optic photoelectric sensor - model E3X-A11 and fiber-optic cable E32-CC200, available from Omron Electronics (Omron Electronic Components, Schaumburg, IL) was used to measure the blower rpm. These sensors have fast response time and adjustable sensing distance. The small size of these sensors allowed them to be accommodated in the limited space available. The output signal was connected to an oscilloscope which displayed the pulse frequency. The rpm value was calculated from the displayed frequency. 


\section{BASE-DESIGN SIMULATION AND EXPERIMENTAL DATA COMPARISON}

The results from the CFD run and the experimental test are given below. A simple sizing convention, to identify the different blower types, is followed. The base-design impeller i.e. the hand held vacuum system blower is specified as 100 units in all three $(X, Y, Z)$ directions. The in-plane directions are represented by $X$ and $Z$ axis, while the impeller axial direction is specified as $\mathrm{Y}$. Any subsequent scaling is mentioned before the direction name, for example the base-design blower, according to this convention, will be named as $100 \mathrm{xz} \_100 \mathrm{y}$. The rpm value at which a blower operates is given after the name. Therefore, a blower named as 70xz_28y@20k has been scaled in the $X$ and $Z$ directions $70 \%$ and in the $Y$ direction by $28 \%$, and the blower was tested at 20,000 rpm.

\section{Blower Efficiency Definitions}

At this point it is necessary to differentiate between the different efficiencies defined in the literature. Equations 15 and 16 (reproduced below) define the energy transfer process in an impeller. Here $\Delta H_{\text {tot }}$ represents the total dynamic head, the other terms have been defined already.

$$
\begin{aligned}
\frac{\dot{W}_{S}}{\dot{m}} & =g \Delta H_{\text {tot }}+\Delta u \\
\text { where } \quad H_{\text {tot }} & =\frac{P_{\text {stat }}}{\rho g}+\frac{V^{2}}{2 g}+Z_{e}
\end{aligned}
$$

As can be seen from Equation 15 not all of the mechanical input energy per unit mass (that is, the shaft power per unit of mass flow rate) ends up as $g \Delta H_{t o t}$, i.e. as useful blower output energy per unit mass. Instead, losses produce an internal energy increase 
$\Delta u$ (manifested as a temperature increase). This fact is obtainable from the Second Law of Thermodynamics and can be represented symbolically as:

$$
g \Delta H_{t o t} \leq \frac{\dot{W}_{S}}{\dot{m}} \text { or } \eta \leq 1
$$

where

$$
\dot{m}=\rho Q
$$

Blower losses are quantified by the overall efficiency $\left(\eta_{\text {overall }}\right)$, which must be less than unity. The overall efficiency can be expressed as:

$$
\eta_{\text {overall }}=\frac{g \Delta H_{\text {tot }} \dot{m}}{\dot{W}_{S}}=\text { Overall Pump Efficiency }
$$

This is the value given in Figures 6 and 8. In the present case, the overall pump efficiency value cannot be measured because there was no way, either in FLUENT or experimentally, to determine both the numerator and denominator. Experimentally, one can determine the shaft power $\dot{W}_{S}$ but cannot measure the total head change. It can be approximated at best. For FLUENT, one can get the average total head change value, but cannot simulate shaft power. This is because the simulation model does not predict the disk friction losses $\left(\dot{W}_{D}\right)$. Therefore, no direct comparison can be drawn between efficiency values given in Figure 6 and the values one gets here. Figure 6 can only act as a benchmark. Mixing simulation values with experimental data to get $\eta_{\text {overall }}$ is not appropriate and these values are not reported. 


\section{Component Efficiencies}

To pinpoint the losses, it is convenient to deal with them in terms of "component efficiencies" (Japikse et al. 1997). For the typical shrouded- or closed-impeller pump shown in Figure 21, Equation 25 can be rewritten as follows:

$$
\eta_{\text {overall }}=\frac{\dot{W}_{I}}{\dot{W}_{S}} \times \frac{g \Delta H_{t o t}\left(\dot{m}+\dot{m}_{L}\right)}{\dot{W}_{I}} \times \frac{\dot{m}}{\dot{m}+\dot{m}_{L}}
$$

where $\dot{W}_{I}$ equals the power delivered to all fluid flowing through the impeller. Also, $\dot{m}_{L}$ is the total external mass leakage past the impeller and back into the impeller inlet. The equivalent volume leakage volume flow-rate is given as $Q_{L}$ (Equation 28).

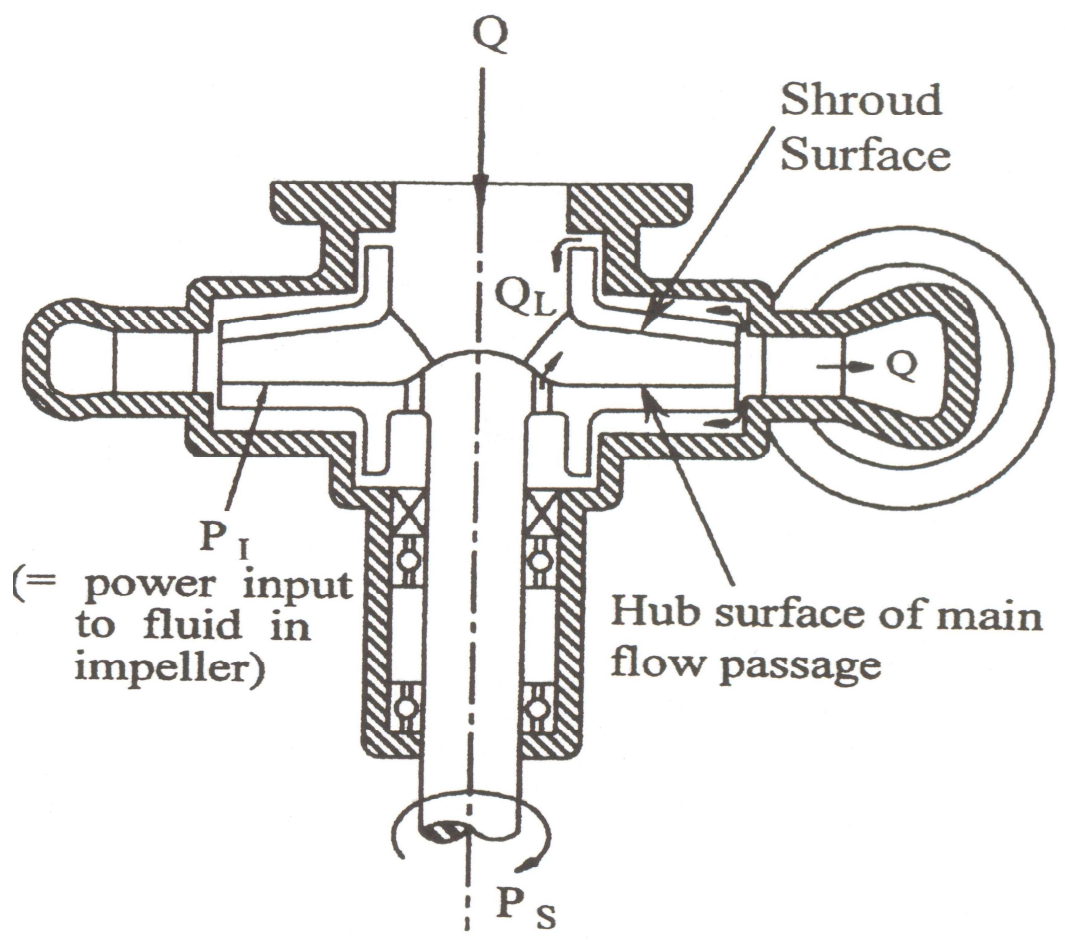

Figure 21: Determining component efficiencies. 
Noting that

$$
\begin{aligned}
\dot{W}_{i} & =g \Delta H_{i}\left(\dot{m}+\dot{m}_{L}\right)=\dot{W}_{I} \\
H_{i} & =\text { ideal head }
\end{aligned}
$$

one may rewrite Equation 26 as follows:

$$
\eta_{\text {overall }}=\frac{\dot{W}_{I}}{\dot{W}_{S}} \times \frac{\Delta H_{t o t}}{\Delta H_{i}} \times \frac{Q}{Q+Q_{L}}=\eta_{m} \times \eta_{H Y} \times \eta_{v}
$$

where,

$$
\begin{gathered}
\eta_{m}=\frac{\dot{W}_{I}}{\dot{W}_{S}}=\frac{\dot{W}_{S}-\dot{W}_{D}}{\dot{W}_{S}} \\
\eta_{H Y}=\frac{\Delta H_{t o t}}{\Delta H_{i}}=\frac{\Delta H_{i}-\sum H_{L}}{\Delta H_{i}} \\
\eta_{v}=\frac{Q}{Q+Q_{L}}
\end{gathered}
$$

The product of all three, i.e. mechanical efficiency $\left(\eta_{m}\right)$, hydraulic efficiency $\left(\eta_{H Y}\right)$, and volumetric efficiency $\left(\eta_{v}\right)$ yields the overall pump efficiency as defined in Equation 26. It reflects the following division of pump losses:

a. Disk Friction Losses $\left(\dot{W}_{D}\right)$ : Disk friction is the external drag on the rotating element due to fluid friction on the outside surfaces of the impeller shroud (Japikse et al. 1997, Gülich et al. 1997). The "mechanical efficiency" is that portion of the shaft power that is delivered to the fluid flowing through the impeller passage. One cannot quantify $\left(\dot{W}_{D}\right)$ through the current FLUENT simulations since the entire blower was not simulated. Only the flow internal to the impeller was considered. The interaction with the blower housing were not included. FLUENT was used to 
calculate the total torque required to overcome the fluid friction inside the impeller and to produce the head and flow rate. This value of $\dot{W}_{I}$ was obtained by integrating the force times the moment arm over the impeller passage surfaces.

b. Hydraulic Efficiency: Hydraulic losses, due to fluid friction, separation, etc. occur in the main flow passages of the pump. The energy loss per unit mass is $g \sum H_{L}=g\left(\Delta H_{i}-\Delta H\right)$. The ratio of actual total output head produced $\Delta H_{\text {tot }}$ to the theoretical head supplied to the fluid $H_{i}$ is the hydraulic efficiency.

c. External Leakage Losses $Q_{L}$ : Leakage losses totaling $Q_{L}$ leak past the impeller and back into the inlet eye. This leakage flow has received its share of the full impeller power $\dot{W}_{I}=\rho g \Delta H_{i}\left(Q+Q_{L}\right)$ delivered to all the fluid $\left(Q+Q_{L}\right)$ passing through the impeller. The leakage power is $\dot{W}_{L}=\rho g \Delta H_{i} Q_{L}$, which is lost as this fluid leaks back to the impeller inlet. The remaining fluid input power is thus $\left(\dot{W}_{I}-\dot{W}_{L}\right)=\rho g \Delta H_{i} Q$, the ratio of this power to the total $\left(\dot{W}_{I}\right)$ being the volumetric efficiency

As already stated, estimating $\eta_{\text {overall }}$ for the present case involves dividing the total head change value obtained from FLUENT, with the shaft power estimated experimentally (Equation 31). Mixing simulation values with experimental data was not deemed appropriated, and therefore, $\eta_{\text {overall }}$ values are not reported. Instead, other efficiency definitions are used as given in next section.

$$
\eta_{\text {overall }}=\frac{\left.g \Delta H_{t o t} \dot{m}\right|_{F L U E N T}}{\left.\dot{W}_{S}\right|_{E X P}}
$$




\section{FLUENT and Experimental Efficiency Definition}

The FLUENT simulations can provide an estimate of $\dot{W}_{I}$ by integrating the force time the moment arm over the impeller passage surfaces. Performance losses due to leakages and hydraulic are not obtained. There is no way to quantify $\dot{W}_{D}$ without the use of rotary transducers, or other setup, to measure shaft power $\dot{W}_{S}$. Neither can the leakage flow rate $Q_{L}$ be estimated. To overcome these limitations, modified efficiency definitions are used through the remainder of this thesis. An important point to note is the mass flow rated used in these definitions.

$$
\left.\begin{array}{l}
\eta_{F L U}=\frac{\text { Static Head Rise }}{\text { Impeller Power }}=\frac{g \cdot \Delta H_{\text {stat }}\left(\dot{m}+\dot{m}_{L}\right)}{\dot{W}_{I}} \\
\eta_{H Y, F L U}=\frac{\text { Total Head Rise }}{\text { Impeller Power }}=\frac{g \cdot \Delta H_{\text {total }} \cdot\left(\dot{m}+\dot{m}_{L}\right)}{\dot{W}_{I}}
\end{array}\right\} \text { Calculated from FLUENT }
$$

- FLUENT Efficiency $\left(\eta_{F L U}\right)$ :This efficiency value is plotted in the simulation result graphs one gets from FLUENT. It gives a measure of the effectiveness of the blower to convert the impeller power $\left(\dot{W}_{I}\right)$ into static head rise.

- Hydraulic Efficiency, FLUENT $\left(\eta_{H Y, F L U}\right)$ : Although, $\left(\eta_{H Y, F L U}\right)$ is predicted using FLUENT simulations it acts as a valuable reference. For a well designed blower hydraulic efficiency values should be high

- Experimental Efficiency $\left(\eta_{\text {EXP }}\right)$ : It is the standard first law definition. It can be defined as $\frac{\text { Energy sort }}{\text { Energy paid for }}$. This value is plotted in the experimental data plots. 
- Blower Efficiency $\left(\eta_{\text {blower }}\right)$ : This is the best representation of actual blower performance independent of the motor used and the velocity head generated.

\section{Hand-held Vacuum System Blower (100xz_100y)}

Blower characteristic curves, calculated using FLUENT, are given in Figure 22. The pressure rise (hence $\Delta H_{\text {stat }}$ ) was obtained by calculating the difference of the areaaverage pressure at the exit and inlet planes. As can be observed, the pressure rise is almost constant over the flow-range simulated. Even though the blower can operate at the design point, it won't be efficient. Further, the design point lies on the positive slope region of the curve. This region can produce unstable blower performance (Karassik et al. 2000, Tuzson 2000).

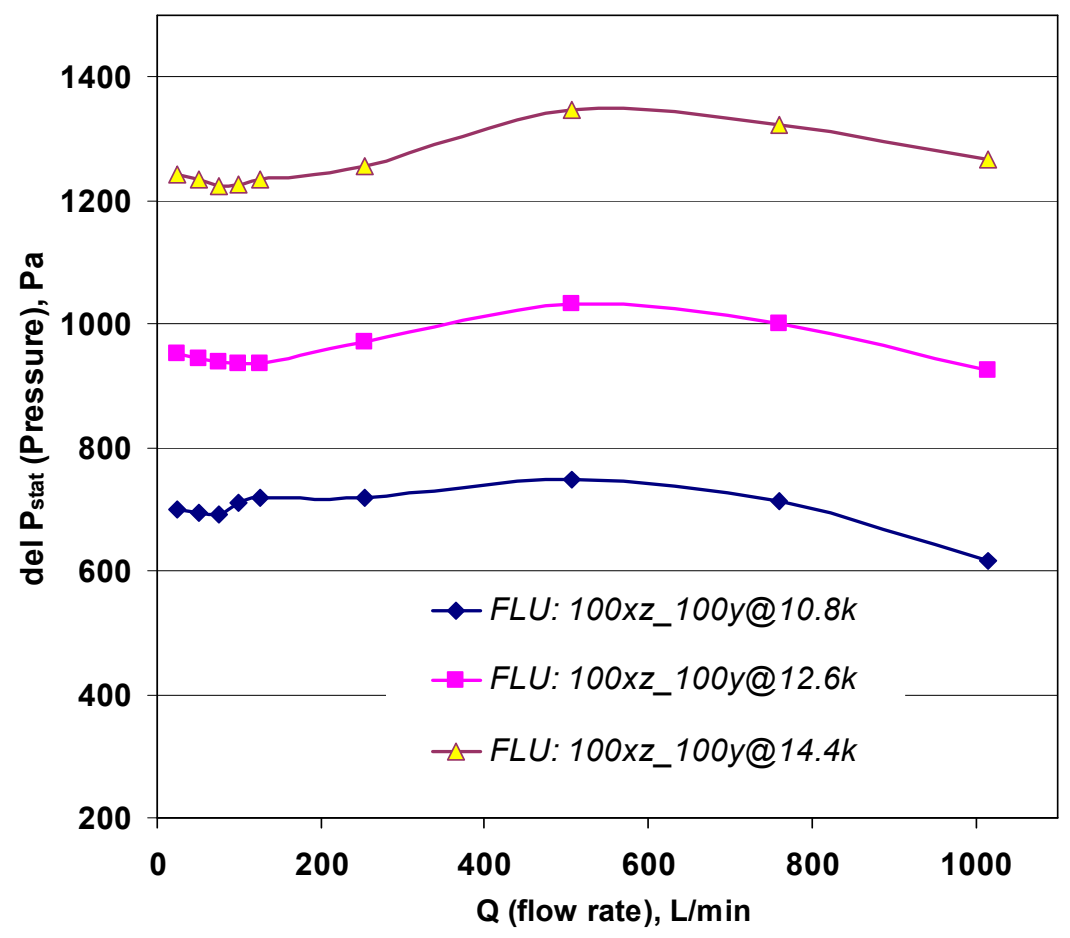

Figure 22: FLUENT performance curves for 100xz_100y blower. 
Experimental results for pressure rise and electrical power consumption are shown in Figures 23 and 24 respectively. The actual head produced by the blower is constant over the flow range. A sample calculation for determining the flow rate from the pressure differential is given in Appendix A. Higher blower rpm could not be used in the experiments as the head produced by the blower at higher rotational rates was more than manometer range. The objective of this initial test was fulfilled with FLUENT showing good agreement with experimental data over the restricted flow range (Figure 25).

The $y^{+}$value distribution for FLUENT simulations was approximately 40 throughout the domain walls. This is acceptable (ERCOFTAC Best Practice Guideline 2000, FLUENT User's Guide 2004). The efficiency value $\left(\eta_{E X P}\right)$ (Figure 26$)$ is less than $10 \%$, this value is very low. Even with an assumed velocity head equal to the static pressure head, the total efficiency value will be under $20 \%$. This low value can be attributed to the low motor efficiency and the blower being operated at a point well below its maximum efficiency flow rate.

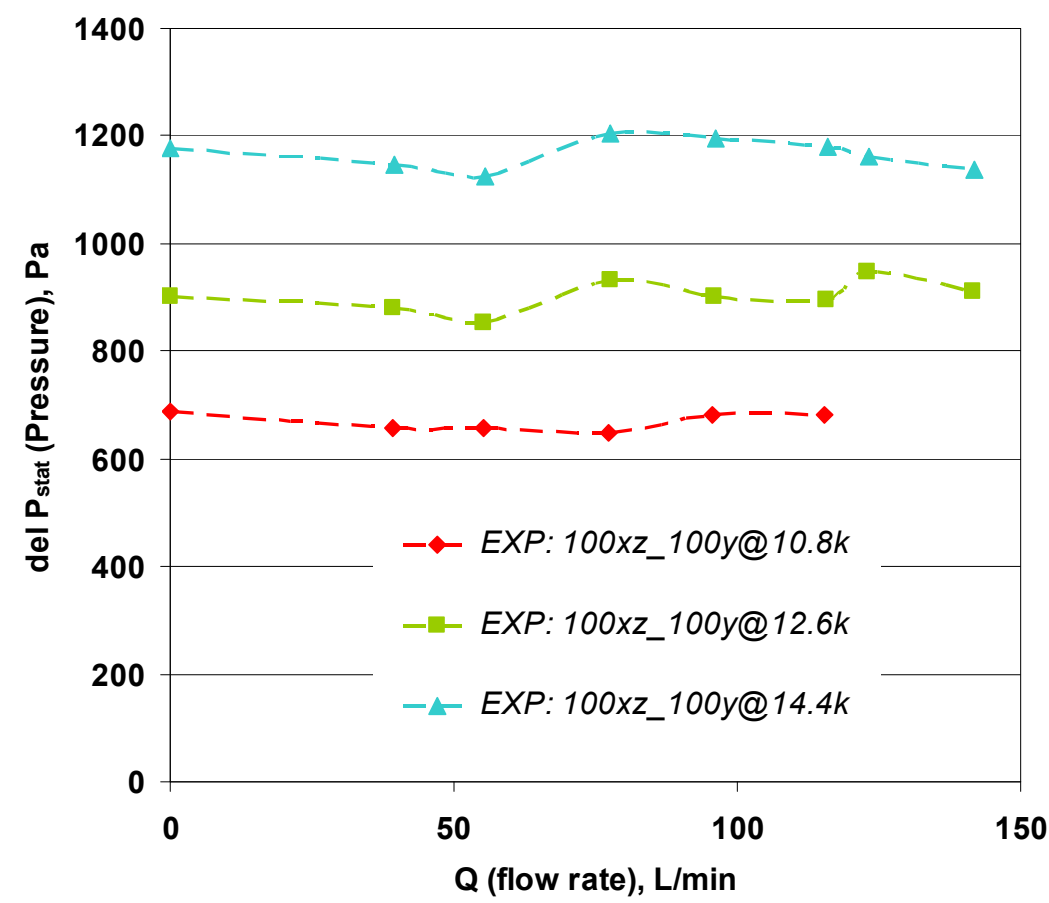

Figure 23: Experimental performance curves for 100xz_100y blower. 


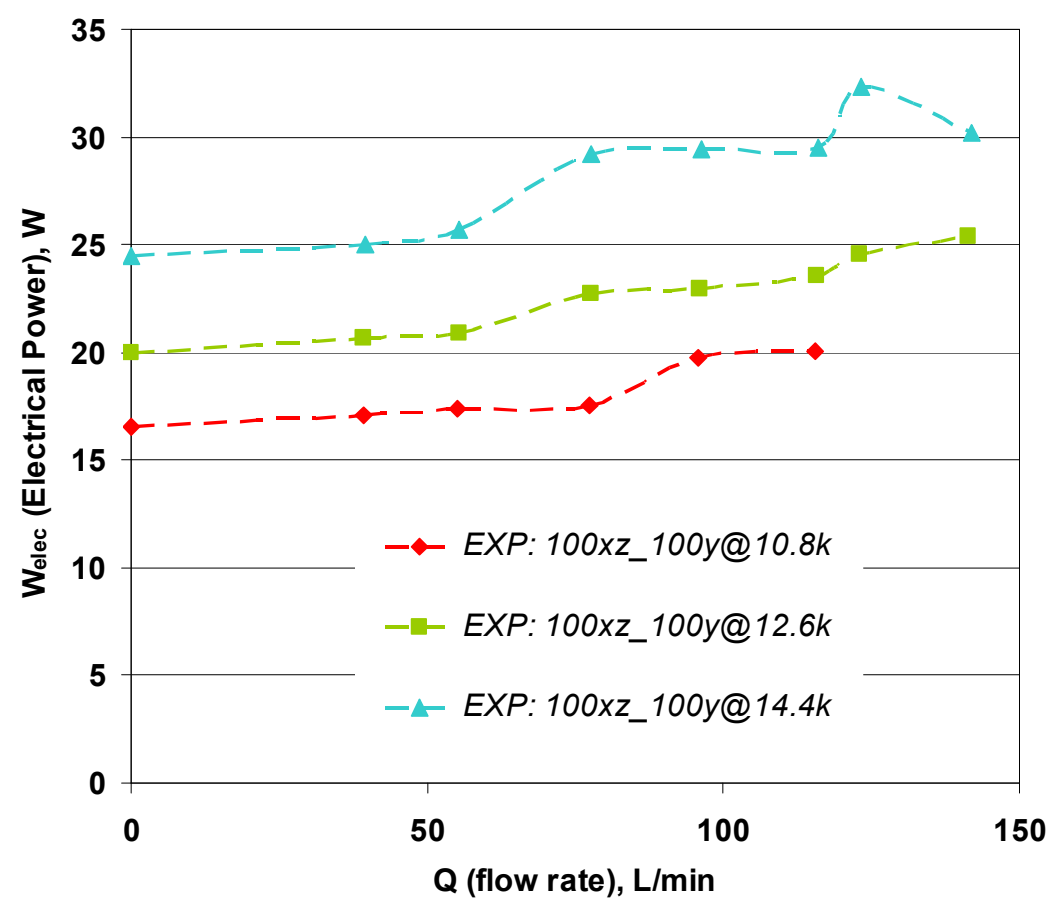

Figure 24: Experimental electrical power consumption curves for 100xz_100y blower.

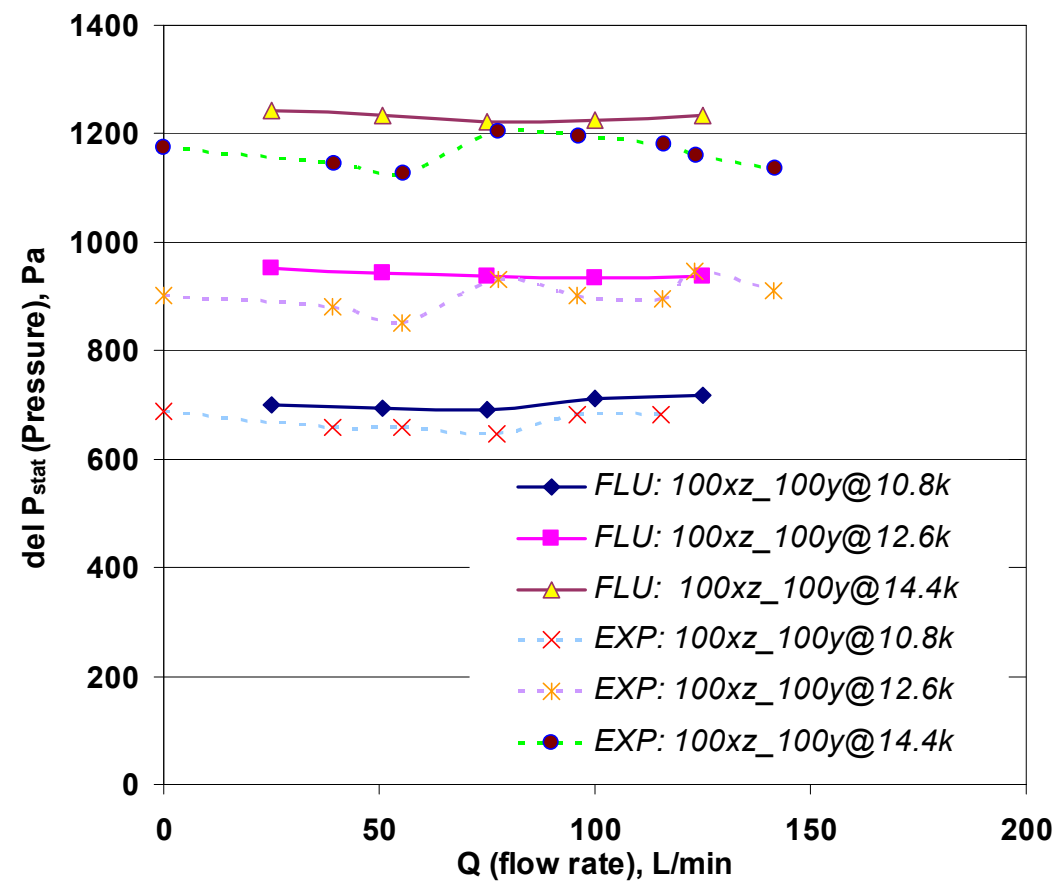

Figure 25: FLUENT -vs. - Experimental data for 100xz_100y blower. 


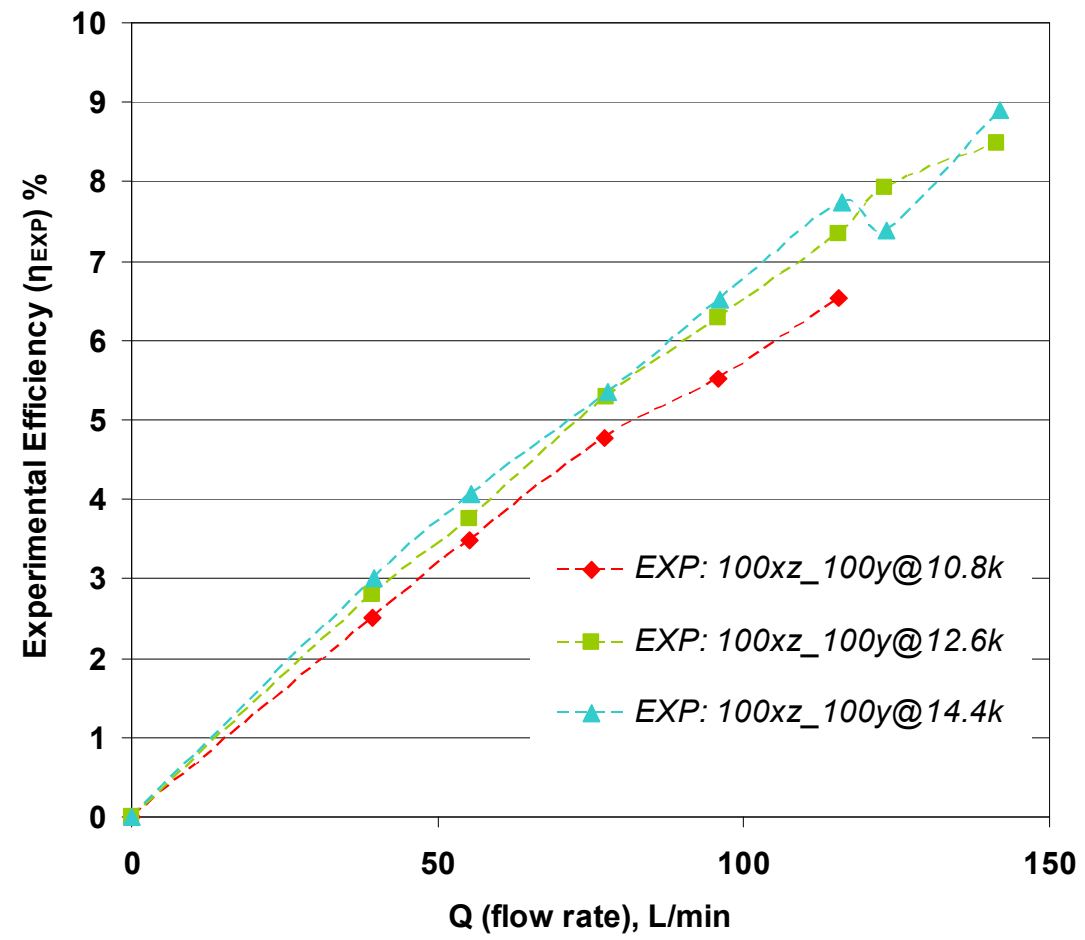

Figure 26: Experimental efficiency $\left(\eta_{E X P}\right)$ values for 100xz_100y blower.

\section{Observation}

CFD results compare well with the experiment measurements. The experimental efficiency $\left(\eta_{E X P}\right)$ of the blower is low (Figure 26); however, this can be accounted by the motor being used to run the blower and the blower being operated at a flow rate well below its maximum efficiency point. Recirculation and separation at low flow rates implies that the blower has been designed for higher flow rates than $100 \mathrm{~L} / \mathrm{min}$. This can also be observed from Figure 22 which shows the maximum pressure value occurring at $600 \mathrm{~L} / \mathrm{min}$. This suggests scaling the blower down by changing the blade height - to affect volume flow rate, and by decreasing the blower diameter - to reduce the head produced. This is done in the subsequent sections. 


\section{FLUENT SIMULATIONS FOR DIFFERENT SCALING CASES}

\section{Fluent Data}

Different scaling was performed in the $X$ and $Z$ and the $Y$ directions. $X$ and $Z$ scaling varied the impeller diameter and hence the pressure-rise across it, whereas the blade height was reduced to change the optimum flow rate. Changing the blade height shifts the blower characteristic curve to the left and causes the peak blower performance to occur at a lower flow rate. Characteristic curves for several different cases are given in Figure 27. Figure 28 presents the efficiency levels $\left(\eta_{F L U}\right)$ predicted by FLUENT. All of these simulations have been performed at $20000 \mathrm{rpm}$; therefore this is not specifically mentioned in the blower name.

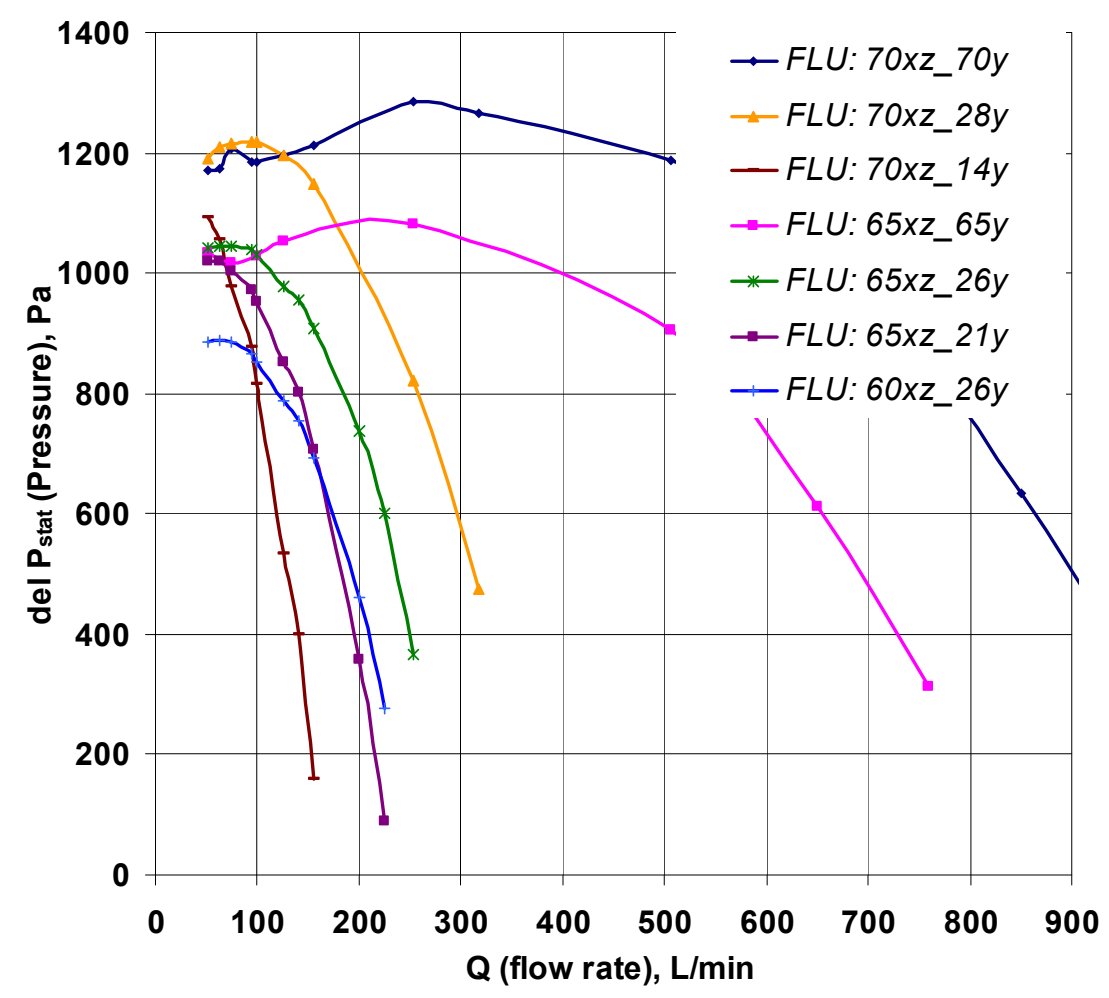

Figure 27: FLUENT performance curve for different-scaled blowers. 


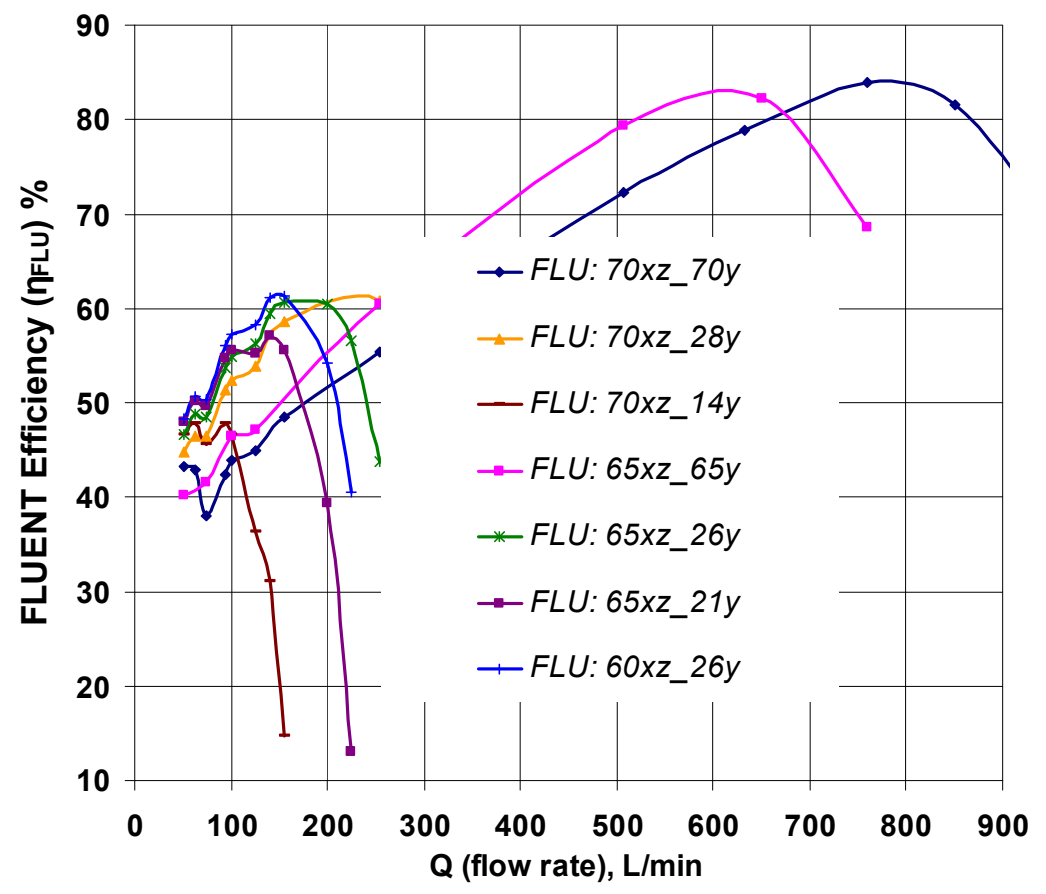

Figure 28: FLUENT efficiency values $\left(\eta_{F L U}\right)$ for different scaled blowers.

Table 3: Sample blower simulation data.

\begin{tabular}{|c|c|c|c|c|c|c|}
\hline \multicolumn{7}{|c|}{ Blower 65xz_21y@20k } \\
\hline Case \# & $\mathrm{Q}(\mathrm{L} / \mathrm{min})$ & $\Delta P_{\text {stat }}(\mathrm{Pa})$ & $T(\mathrm{~N} . \mathrm{m})$ & $\dot{W}_{I}(\mathrm{~W})$ & $\dot{m} . g . \Delta H_{\text {stat }}(\mathrm{W})$ & $\eta_{F L U} \%$ \\
\hline 1 & 50.7 & 1019.0 & 0.00009 & 1.79 & 0.86 & 48.04 \\
\hline 2 & 62.4 & 1018.8 & 0.00011 & 2.10 & 1.05 & 50.10 \\
\hline 3 & 75 & 1003.5 & 0.00013 & 2.48 & 1.23 & 49.60 \\
\hline 4 & 93.7 & 973.3 & 0.00015 & 2.77 & 1.53 & 54.67 \\
\hline $\mathbf{5}$ & $\mathbf{1 0 0}$ & $\mathbf{9 5 4 . 0}$ & $\mathbf{0 . 0 0 0 1 5}$ & $\mathbf{2 . 9 0}$ & $\mathbf{1 . 6 1}$ & $\mathbf{5 5 . 5 6}$ \\
\hline 6 & 125 & 851.4 & 0.00017 & 3.20 & 1.76 & 55.19 \\
\hline 7 & 140 & 801.6 & 0.00017 & 3.25 & 1.86 & 57.10 \\
\hline 8 & 156 & 706.3 & 0.00017 & 3.29 & 1.83 & 55.62 \\
\hline 9 & 200 & 357.4 & 0.00016 & 3.00 & 1.18 & 39.39 \\
\hline 10 & 225 & 90.6 & 0.00014 & 2.60 & 0.34 & 13.04 \\
\hline
\end{tabular}


A sample of the data from the FLUNET simulations is given above (Table 3). From Figure 26, the effects of blower diameter and blade height are clearly indicated. The efficiency value of the impeller decreases as the impeller is made smaller. However, the overall power required to produce the $1000 \mathrm{~Pa}$ head at $100 \mathrm{~L} / \mathrm{min}$ is less for the small, less efficient blowers than the larger blowers which are more efficiency but require more power to operate at the off design conditions. Trying to balance the rate of pressure decrease and Best Efficiency Point (BEP) effect, it appears that blower 65xz_26y will give optimum efficiency and a good performance safety margin (Table 4) under 20,000 rpm. Blower size65xz_21y could also be used, but the pressure rise versus flow rate curve has a very steep slope providing very small margin for error and it needs a higher rpm than 20,000 to get the pressure rise (Table 4). The characteristic curve and the efficiency value are given below (Figure 29) for the selected blower.

Table 4: Efficiency values for different scaled blower at design flow rate.

\begin{tabular}{|c|c|c|c|c|}
\hline $\begin{array}{c}\text { Planar Scaling } \\
(\mathrm{xz})\end{array}$ & $\begin{array}{c}\text { Axial Scaling } \\
(\mathrm{y})\end{array}$ & $\mathrm{RPM}$ & $\Delta P_{\text {stat }}(\mathrm{Pa})$ & $\eta_{F L U} \%$ \\
\hline $70 \mathrm{xz}$ & $70 \mathrm{y}$ & 18500 & 1028.5 & 31.2 \\
\hline $70 \mathrm{xz}$ & $28 \mathrm{y}$ & 18500 & 1025 & 44.96 \\
\hline $70 \mathrm{xz}$ & $14 \mathrm{y}$ & 21500 & 1033.2 & 52.6 \\
\hline $65 \mathrm{xz}$ & $65 \mathrm{y}$ & 19500 & 1031.54 & 48.33 \\
\hline $65 \mathrm{xz}$ & $26 \mathrm{y}$ & 20000 & 1006.5 & 38.2 \\
\hline $65 \mathrm{xz}$ & $21 \mathrm{y}$ & 20500 & 1030.5 & 54.5 \\
\hline $60 \mathrm{xz}$ & $26 \mathrm{y}$ & 21500 & 1011.44 & 55 \\
\hline
\end{tabular}




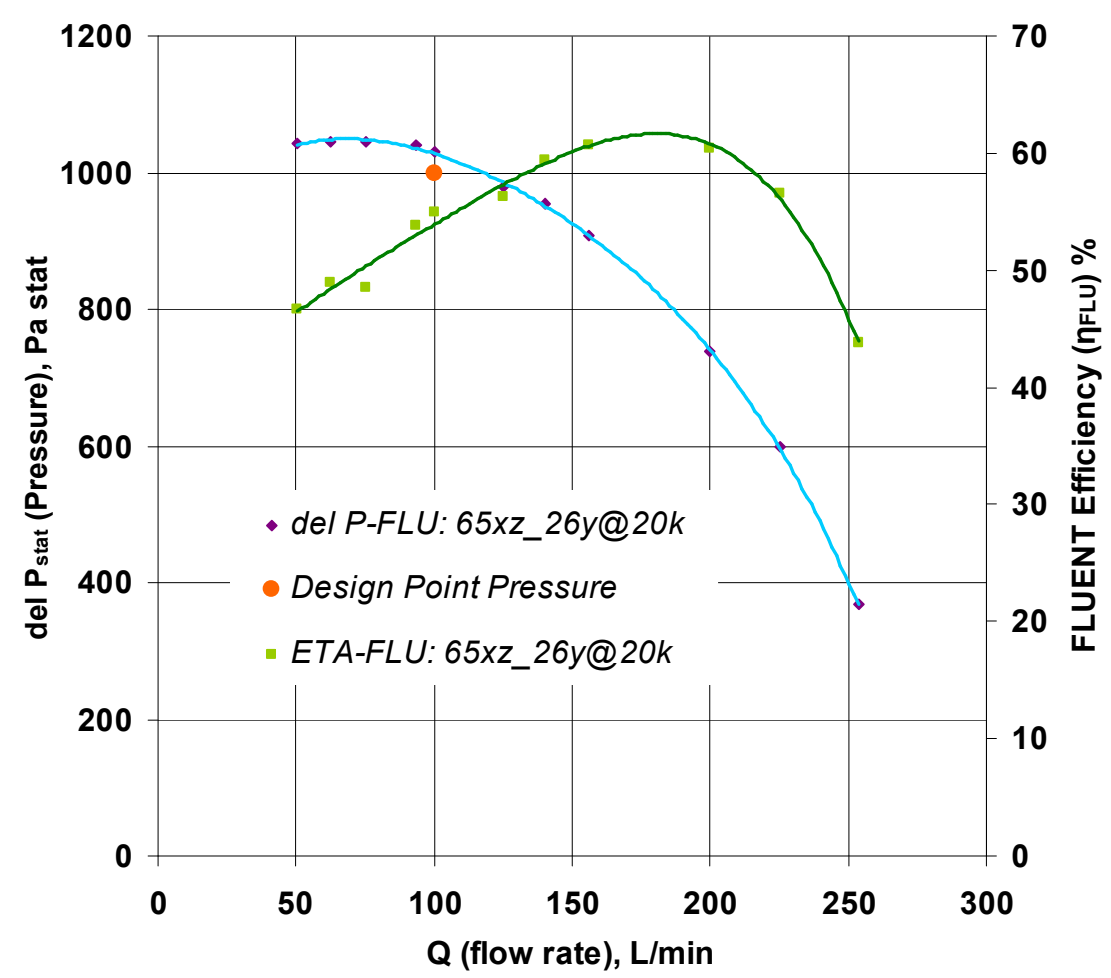

Figure 29: FLUENT performance curves for 65xz_26y@20k blower.

FLUNET gives the impeller power $\dot{W}_{I}$ as $2.9 \mathrm{~W}$. Allowing for additional losses due to the blower housing, the shaft power $\dot{W}_{S}$ should be less than $4 \mathrm{~W}$. Experiments were carried out with this blower, and the CFD model was validated. Before considering that, it will be interesting to look at the non-dimensional results for the different blower sizes simulated with FLUENT.

\section{Non-dimensional Study for Blower Simulation Results}

In the present study, non-traditional fan-scaling laws have been used to decide upon the impeller radius $\left(R_{2}\right)$ and the blade height $\left(b_{2}\right)$. The conventional fan-scaling laws are valid (to an extent) when the scaling is uniform in all three directions. In fact, uniform 
scaling in all three directions is an underlying assumption in the fan laws (Japikse et al. 2000). This is verified by the fact that in the traditional fan-laws the blower dimension is represented as a single length scale $\left(R_{2}\right)$ (Equations 2 and 5), and all other dimensions scale accordingly.

$$
\begin{gathered}
Q_{s}=\frac{Q}{\Omega R_{2}^{3}} \\
\psi=\frac{g \Delta H_{t o t}}{\left(\Omega R_{2}\right)^{2}}
\end{gathered}
$$

When dealing with different axial and planar scaling, the scaling law must be modified. This was done by incorporating the blade height at the exit $\left(b_{2}\right)$ into the scaling equations. The approach was to keep the exponent of the length term same i.e. the sum of the exponents for $R_{2}$ and $b_{2}$ should be equal to 3 for flow coefficient (equation 2); and equal to 2 for the head coefficient term (equation 5). In the present case, since we are concerned with static pressure rise value, it was decided to use only the static head term instead of the total head term as done for conventional scaling laws (Equation 34 and 35).

$$
\begin{aligned}
& Q_{s, \text { mod }}=\frac{Q}{\Omega \cdot R_{2}{ }^{x} \cdot b_{2}{ }^{y}} \\
& x+y=3 \\
& \text { and, } \quad \psi_{\bmod }=\frac{g \Delta H_{s t a t}}{\Omega^{2} \cdot R_{2}{ }^{i} \cdot b_{2}{ }^{j}} \\
& i+j=2
\end{aligned}
$$

Estimated values for the exponents $x, y, i, j$ were chosen. Simulation data for the different blower sizes were non-dimensionalized according to Equations 34 and 35. The process was iterated upon till an acceptable data overlap was obtained. The results are shown below in Figure 30; all data points are at 20000 rpm except for the 100xz_100y data points which are at $14400 \mathrm{rpm}$ and provide a good cross check for scaling at different rpm values. 


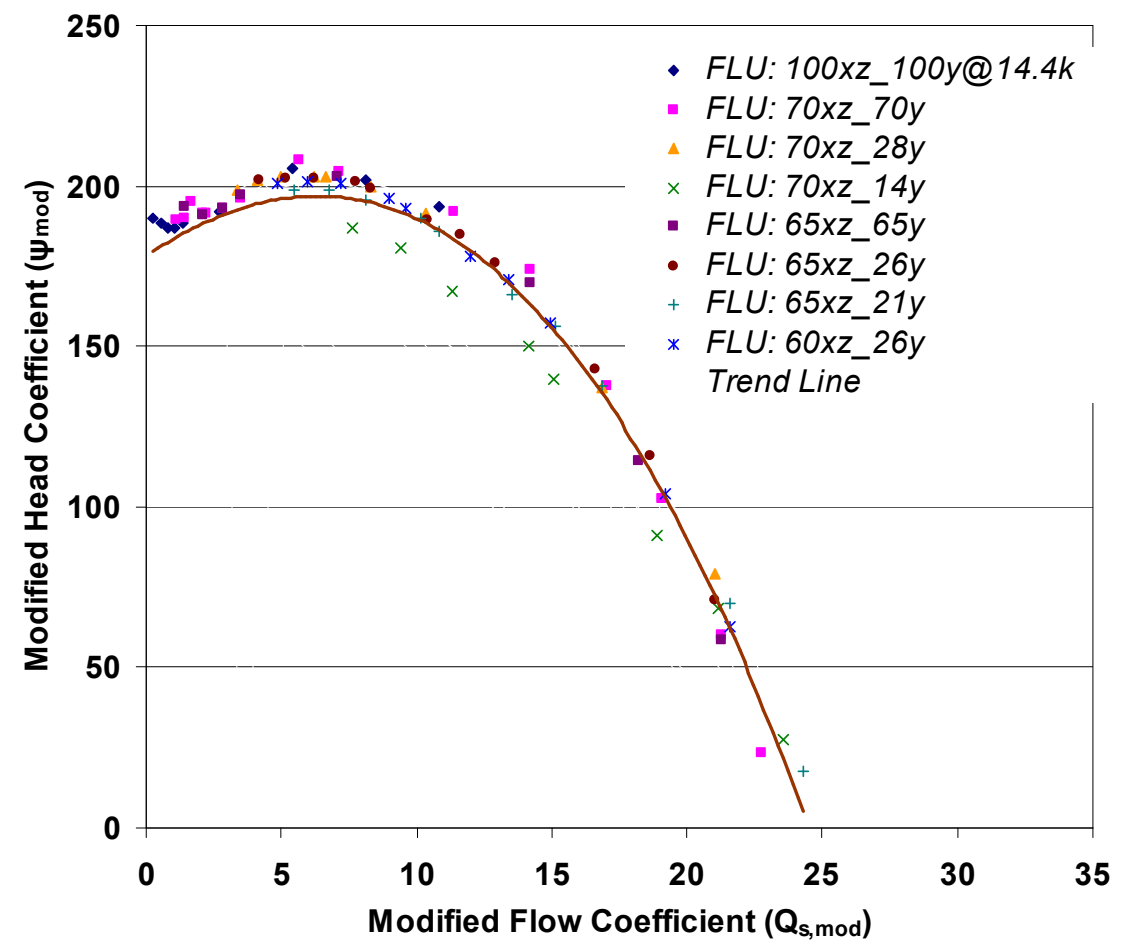

Figure 30: FLUENT non-dimensional modified head coefficient curve.

From the analysis, the modified scaling laws are given as (Equation 36)

$$
\begin{aligned}
& Q_{s, \text { mod }}=\frac{Q}{\Omega \cdot R_{2}{ }^{1.815} \cdot b_{2}{ }^{1.185}} \\
& \psi_{\text {mod }}=\frac{g \Delta H_{\text {stat }}}{\Omega^{2} \cdot R_{2}{ }^{1.965} \cdot b_{2}{ }^{0.035}}
\end{aligned}
$$

Table 5: Exponents for radius and blade width.

\begin{tabular}{|c|c|c|}
\hline$\frac{\text { Equation } \rightarrow}{\text { length factor } \downarrow}$ & Flow-coefficient & Head-coefficient \\
\hline Radius $\left(R_{2}\right)$ & 1.815 & 1.965 \\
\hline Blade width $\left(b_{2}\right)$ & 1.185 & 0.035 \\
\hline
\end{tabular}


The exponents (Table 5) show that the pressure produced by the impeller is a weak function of the blade height. At the same time, blade height has a significant effect on the flow rate through the impeller. This is expected. Another fact is that the optimum flow rate through an impeller is affected by impeller radius. Equation 36 minimizes the use of FLUENT for the present case as the characteristic curve for virtually any blower size can be estimated using the modified scaling laws. Non-dimensional study also illustrates the consistency of the FLUENT simulation results. 


\section{EXPERIMENTAL VALIDATION FOR 65XZ_26Y@20K BLOWER}

Experimental results for the selected fan,65xz_26y@20k based on the initial FLUENT simulations with non-equilibrium wall function approach, are given below.

\section{Motor Selection}

Selection of a high efficiency motor is essential to achieve high overall performance. Due to the high speeds involved, it was decided to employ a brushless D.C. motor. Brushless D.C. motors are often used in fan applications due to their speed controllability, high efficiency, and long life time (Lelkes 2004). Since the blower is to be designed for long life time, using brushless D.C. motor is advantageous as the only wearing part is the bearing system. These motors have a higher efficiency than induction motors (Lelkes 2004); therefore their temperature remains lower than that of induction motors. A lower operating temperature means a lower bearing temperature and hence, a longer life time expectancy.

A general survey was done and two motors were finalized. These motors were provided by MicroMo Electronics, Inc (Clearwater, FL). The group specializes in the design, assembly and application of high precision, miniature D.C. drive systems. The motors chosen were:

- $\quad 1628 \_024 \mathrm{~B}$ with a nominal voltage of $24 \mathrm{~V}$ and maximum motor efficiency $\left(\eta_{\text {motor }}\right)$ of $68 \%$ (Appendix B, data sheet)

- 2036_024 B with a nominal voltage of $24 \mathrm{~V}$ and maximum motor efficiency $\left(\eta_{\text {motor }}\right)$ of $69 \%$ (Appendix B, data sheet) 
Both motors possess similar performance levels, however, the 2036_024 B with a slightly larger-sized shaft was chosen as it has less lateral movement at $20000 \mathrm{rpm}$. The MicroMo drive electronics BLD 5604 was chosen for motor control.

\section{Blower Manufacturing}

Rapid prototyping (RP) was used to build and test the different blower sizes. The process used was Stereolithography (SLA) which produces physical, three dimensional objects. The services were provided by Stereolithography.com (Tulsa, OK). A stereolithography machine uses a computer-controlled laser to cure a photo-sensitive resin, layer-by-layer, to create the 3D part. Stereolithography is fast, allows prototypes to be made in a matter of days, and the complexity of the model is seldom a factor. SLA is a relatively expensive process but provides high tolerance levels, the machines used had a vertical resolution of $0.0025 \mathrm{~mm}\left(0.0001^{\prime \prime}\right)$ and a positional accuracy of $0.0076 \mathrm{~mm}\left(0.0003^{\prime \prime}\right)$ (http://www.stereolithography.com). It was observed that the prototypes undergo some shrinkage, especially in the axial direction, which reduces the blade height. To avoid this problem, the CAD model was given a10\% size-compensation in the axial direction. This is a precaution to be kept in mind for future work.

\section{Modified Test-rig}

The test rig was modified to fit the small blower size. The main challenge faced was to control the tip clearances $(\delta)$ accurately. Tip clearance has a significant impact on the overall performance observed (Karassik et al. 2000, Engeda 1995, Wood et al. 1965).

Ideally, the blower needs to be sealed at three places: the eye $\left(\delta_{\text {inlet }}\right)$, the exits at the shroud surface $\left(\delta_{\text {shroud }}\right)$, and the hub surface $\left(\delta_{\text {hub }}\right)$. In the present case, since the exit air is discharged and not collected, the leakage at the hub surfaces is not critical. On the other hand, leakage flow coming back to the blower inlet through the shroud and eye sections needs to be controlled. This recirculating flow affects the pressure head produced by the 
impeller. Leakage flow does not add to the flow rate coming through the venturi, as it is fluid recirculated from the pump exit to its inlet. This effectively increases the flow rate through the impeller compared to the flow rate produced by the blower. This increased total flow rate causes the pressure produced by the impeller to drop. But, at the same time, since the leakage flow never passes through the venturi, the flow rate reading observed across the venturi does not change. Effectively, the pressure produced for a given flow rate reading across the venturi drops, or the characteristic curve shifts down. Therefore, the clearances are essential in the efficient operation of the blower and must be monitored. The motor was mounted in a base plate and the impeller seated in a stepplate (Figure 31). To account for the tip clearance effects, these values are mentioned in the data presented.

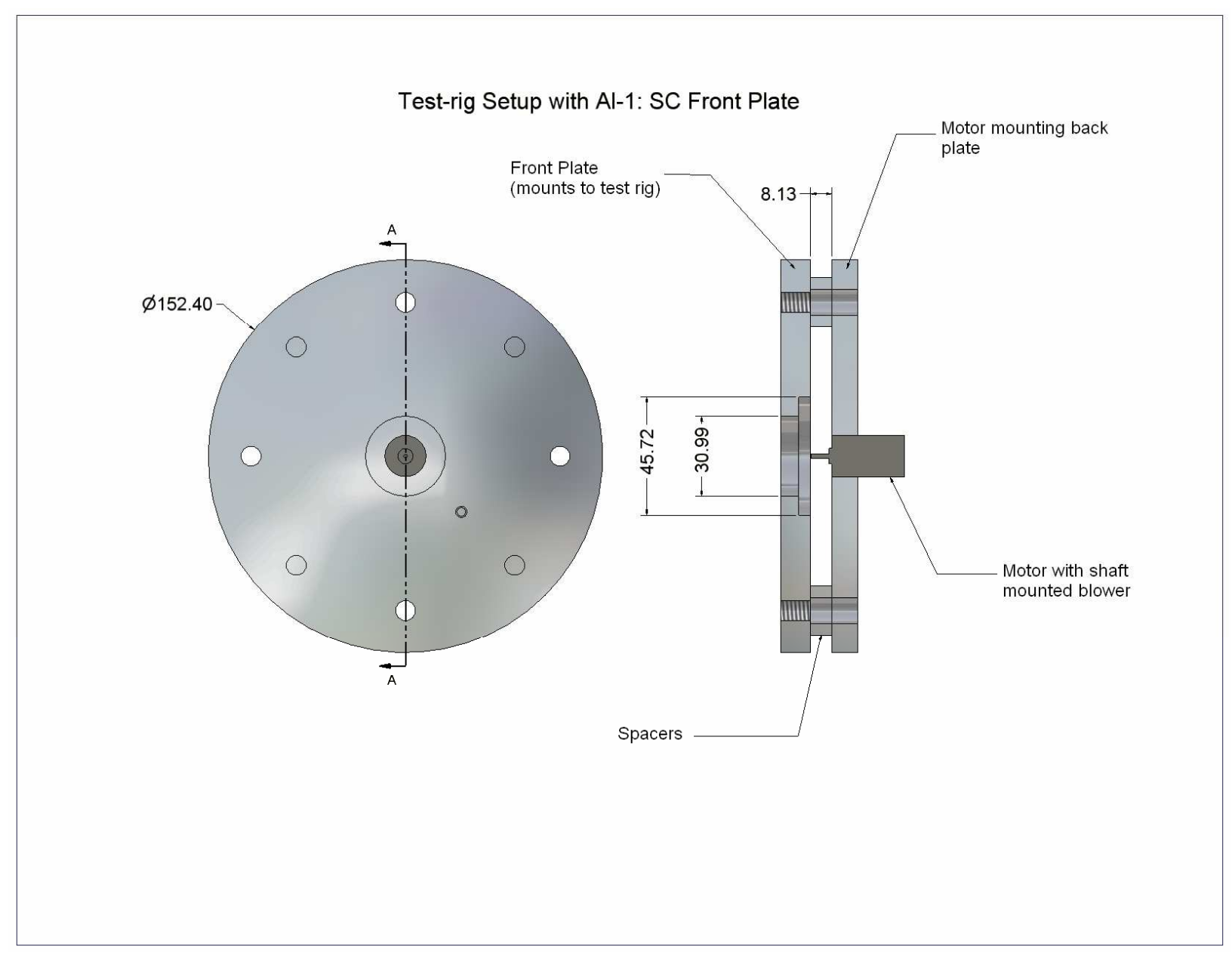

Figure 31: Test rig setup with Al-1: SC front plate (dimensions in $\mathrm{mm}$ ). 


\section{Experimental Data}

The initial blower was tested but the data were not at all in agreement with the simulations. This was attributed to the decreased blade height that resulted from the shrinkage during the Rapid Prototyping (RP) process. This brought out the point that blower dimensions, especially the blade width, are not accurate due to certain limitations of the RP process. A machined blower might be a future recommendation once the design is finalized.

Blower size 65xz_26y had an average (three readings) shroud diameter $\left(D_{2}\right)$ of $1.753 "$, and an average impeller inlet diameter $\left(d_{i n}\right)$ of 1.200" after machining. Two different front plates were used as given below:

- Aluminum Plate \# 1 with Small Clearances $(A l-1: S C)$

- Plate inlet diameter $\left(d_{\text {in, plate }}\right)=1.212^{\prime \prime}$

- Plate shroud diameter $\left(d_{2, \text { plate }}\right)=1.792 "$

- Aluminum Plate \# 2 with Bigger Clearances $(A l-2: B C)$

- Plate inlet diameter $\left(d_{\text {in,plate }}\right)=1.212^{\prime \prime}$

- Plate shroud diameter $\left(d_{2, p l a t e}\right)=1.914 "$

Based on these values the radial clearances one gets are:

- $\delta_{\text {inlet }}=0.006^{\prime \prime}$

- $\quad \delta_{\text {shroud }}=0.020$ for $A l-1: S C$

- $\delta_{\text {shroud }}=0.080^{\prime \prime}$ for $A l-2: B C$

Shroud clearance values for $A l-2: B C$ are relatively high (Karassik et al. 2000) and should affect the performance. For $A l-1: S C$ plate the values are acceptable, but better results can be expected on reducing these clearances. Results for these two plates are given next (Figures 32 and 33). Electrical power consumption measured is shown in Figure 34, with comparison to FLUENT simulations given in Figure 35. 


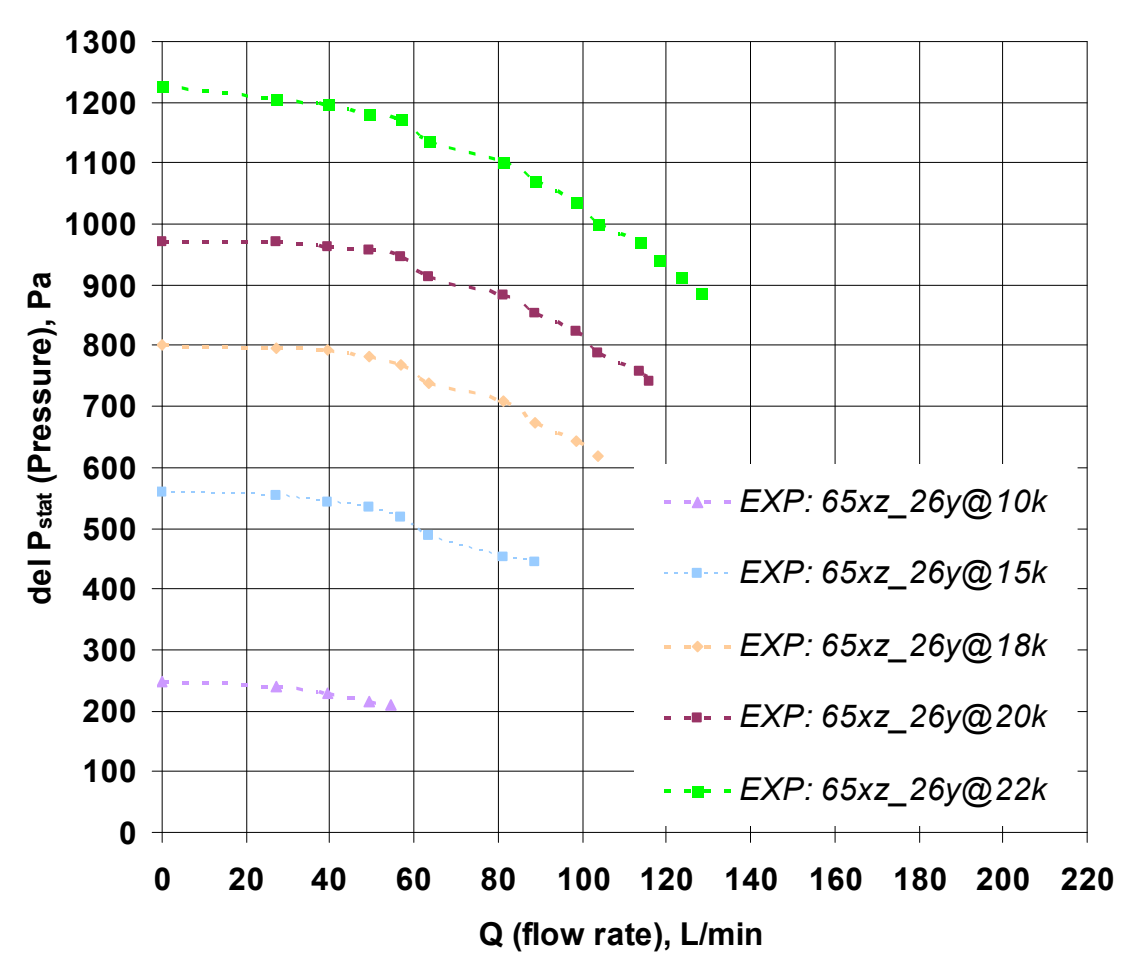

Figure 32: Experimental performance curves for $65 \mathrm{xz}_{-} 26 \mathrm{y}$ blower with Al-2: BC plate.

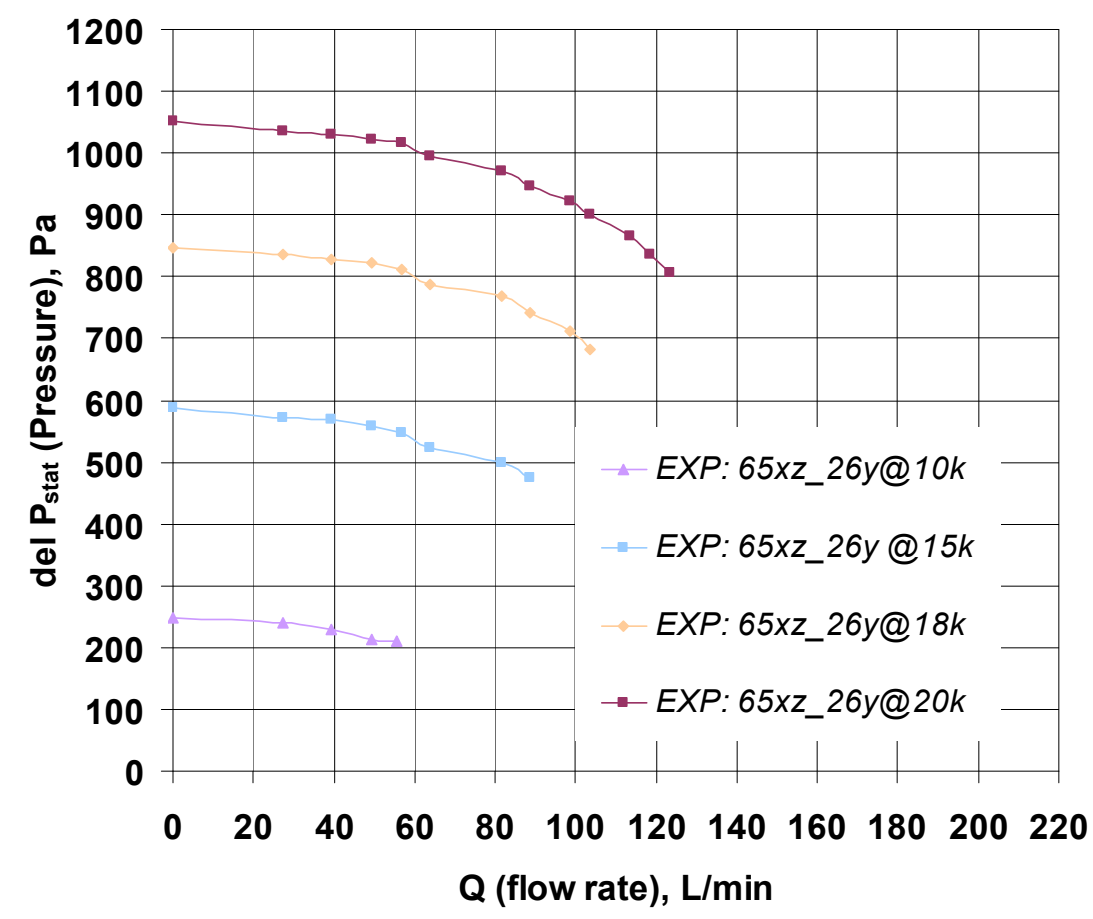

Figure 33: Experimental performance curves for 65xz_26y blower with Al-1: SC plate. 


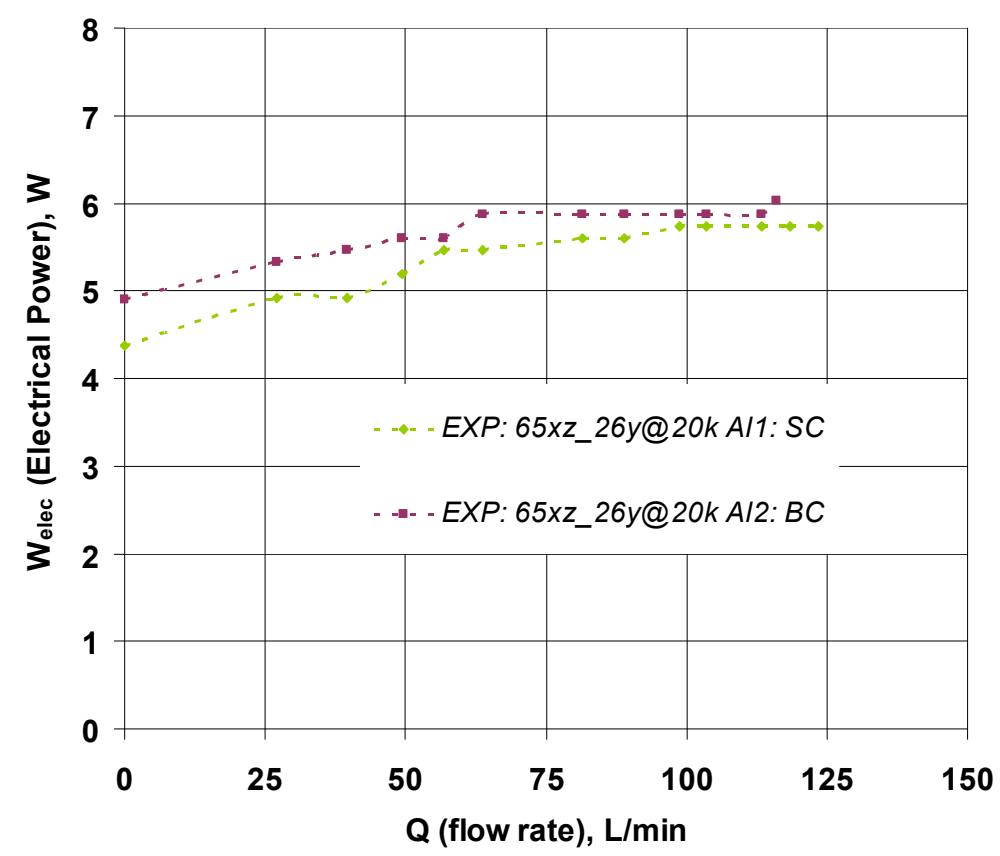

Figure 34: Experimental electrical power consumption curves for 65xz_26y blower with different front plates.

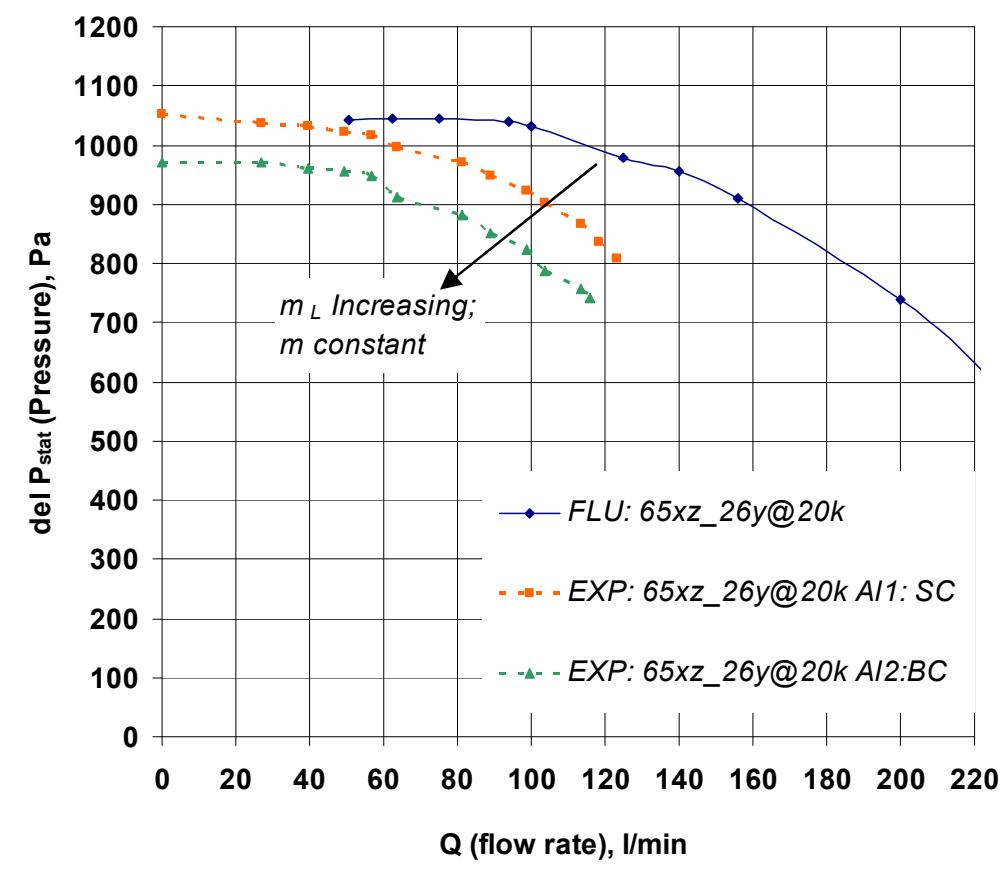

Figure 35: Comparison between performance curves for FLUENT, Al-1: SC, and Al-2: $\mathrm{BC}$ front plates. 
The difference in the performance due to the tip clearance is clearly indicated. The performance curves shift left as the clearance values are increased. This is because $\dot{m}_{L}$ increases from zero for simulations to a finite value as the clearances increase. Hence impeller $\dot{m}_{\text {total }}$ increases and curve shifts left.

FLUENT over-predicts the performance as compared to experimental results (Figure 33). Experimentally, with smaller clearances one gets a pressure rise of $921 \mathrm{~Pa}$ as compared to a predicted value of $1030 \mathrm{~Pa}$. This represents a percentage error of $11 \%$. This might have been acceptable but for the fact that at $921 \mathrm{~Pa}$ the blower is not meeting the design requirements. The $11 \%$ difference can be accounted for by the leakage flow (to a small extent), and by the over-prediction of pressure by the FLUENT model being used. This can be caused by the wall function used, as mentioned earlier. The next logical step is to use enhanced wall treatment approach which is more robust and accurate for flows with high body force and swirl effects (FLUENT User's Guide 2004).

\section{Observations and Conclusions}

Important conclusions that can be drawn from this section are:

- Tip clearance plays an important part in blower performance

- FLUENT predictions match qualitatively with the experimental results

- FLUENT model over-predicts the experimental results gotten with Al-1:SC relatively small clearances plate by $11 \%$

- Blower size 65xz_26y@20k does not meet the design requirements in terms of pressure rise.

- FLUENT model needs to be modified to improve the match to the experimental results

- Since blower size 65xz_26y@20k cannot meet the design specification a bigger blower must be used. 


\section{ENHANCED WALL TREATMENT}

The difference in the two wall treatments can be understood in terms of the non-

dimensional distance, $y^{+}=\frac{\rho \cdot u_{\tau} \cdot y}{\mu}$ to the wall from the first grid cell. The wall function formulation requires a $y^{+} \approx 30$ while for enhanced wall treatment requires $y^{+} \approx 1$ (FLUENT User's Guide 2004). The FLUENT manual says that a higher $y^{+}$is acceptable as long as it is well inside the viscous sublayer $\left(y^{+}<4\right.$ to 5$)$. In the present case, the $y^{+}$values were kept in this range. The total number of grid cells was approximately 150,000 and this is an order of magnitude higher than for the previous wall function approach $(14,000)$. Adaptive meshing available in FLUENT was used to progressively refine the grid.

\section{Enhanced Wall Treatment FLUENT Simulations for 65xz_26y@20k Blower}

Simulation data for 65xz_26y@20k blower with the enhanced wall treatment are given below (Figure 36). Also shown is the experimental curve. Predictions by the enhanced wall treatment are conservative. The percentage error is less for the enhanced wall treatment approach (Figure 37). Nonetheless, the blower size chosen is not appropriate to get to the design point. Enhanced wall treatment will be used to determine a new size estimate for the blower. Before that, a conservative estimate of blower efficiency is given next. 


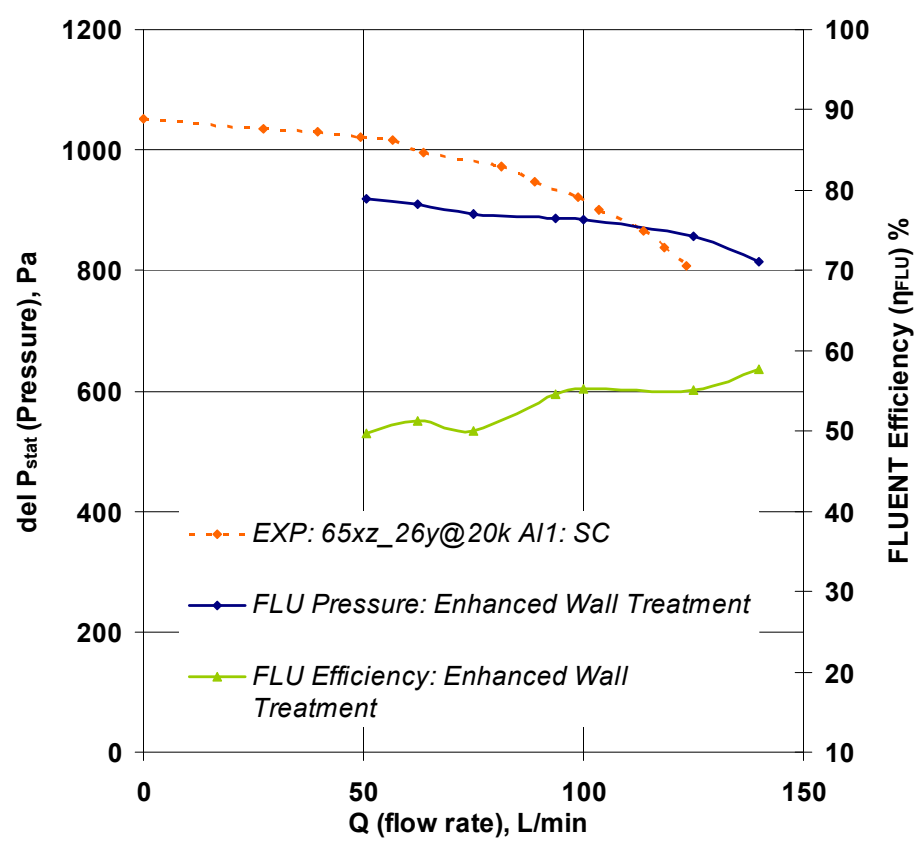

Figure 36: Performance comparison for 65xz_26y blower: Enhanced wall treatment -vs. - experimental results with Al-1: SC front plate

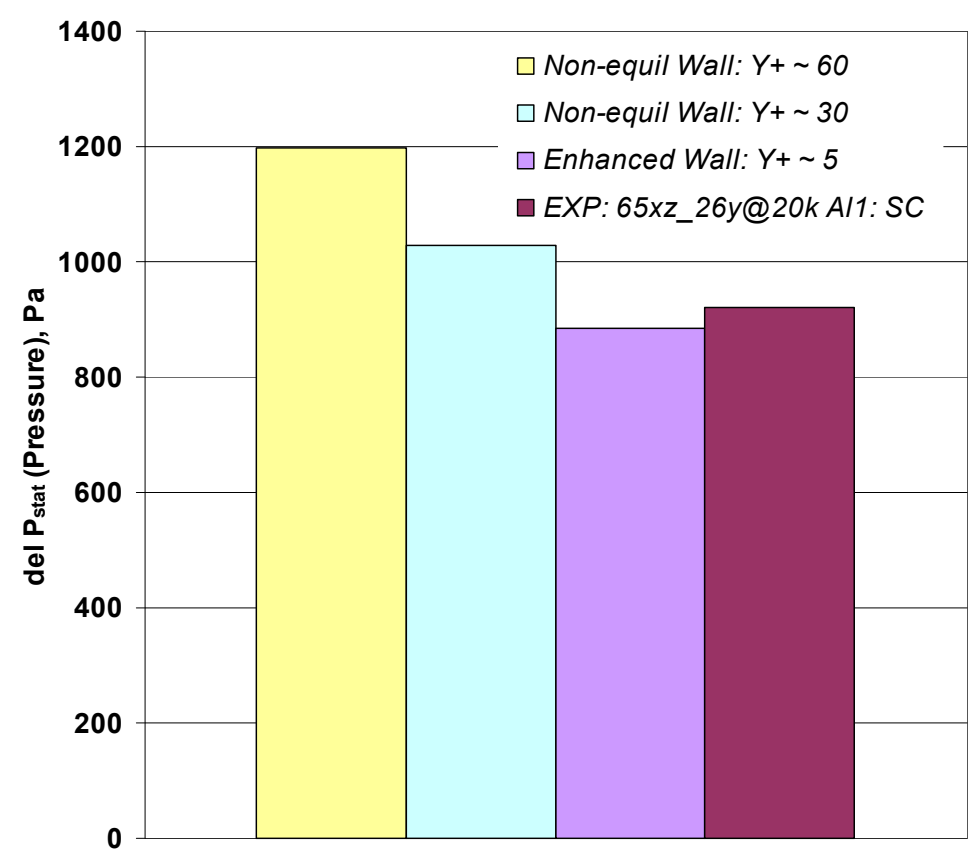

Figure 37: FLUENT static pressure rise value for 65xz_26y blower for different wall treatments and comparison with Al-1: SC experimental data. 


\section{Conservative Efficiency Estimates for 65xz_26y@20k Blower}

Estimates of the different blower efficiencies, namely, $\eta_{H Y, F L U}$ and $\eta_{F L U}$ from FLUENT (Equation 32), and $\eta_{E X P}$ and $\eta_{\text {blower }}$ from experiments (Equation 33) are given below. Following relations are used:

$$
\begin{aligned}
& \dot{W}_{S}=\eta_{\text {motor }} \cdot \dot{W}_{\text {elec }} \\
& \eta_{\text {motor }}=\text { Maximum motor efficiency (from data sheet) }
\end{aligned}
$$

Table 6 shows these values for the $100 \mathrm{~L} / \mathrm{min}$ flow rate case. For the simulations, the efficiencies are based upon total flow rate, i.e. $\dot{m}+\dot{m}_{L}$. For the measurements, $\dot{m}_{L}$ is not known and only $\dot{m}$ is used in the calculation, hence the flow rate in the impeller is undetermined for the measurements. The blower specific speed at the design flow rate is $N_{s,(U S)}=1096$ (Equation 38)

$$
N_{s,(U S)}=\frac{N(\mathrm{rpm}) \sqrt{Q\left(\mathrm{GPM}_{\mathrm{US}}\right)}}{\left[\Delta H_{\text {tot }}(\mathrm{ft})\right]^{3 / 4}}=\frac{(20000 \mathrm{rpm}) \cdot\left(26 \cdot 4 \mathrm{GPM}_{\mathrm{US}}\right)^{0.5}}{(425.8 \mathrm{ft})^{3 / 4}}=1096 \frac{{\mathrm{rpm} \cdot \mathrm{GPM}_{\mathrm{US}}}_{\mathrm{ft}^{0.75}}}{\mathrm{ft}^{0.5}}
$$

Table 6: Blower efficiency estimates at design flow rate.

\begin{tabular}{|c|}
\hline Blower Size 65xz_26y@20k \\
\hline$\dot{W}_{\text {elec }}=5.73 \mathrm{~W}$ \\
\hline$\eta_{\text {motor }}=69 \%$ (best, data sheet) \\
\hline$\left.\Delta P_{\text {stat }}\right|_{E X P}=921.6 \mathrm{~Pa}$ \\
\hline$\dot{W}_{S}=3.95 \mathrm{~W}$ \\
\hline$\left.g \Delta H_{\text {stat }}(\dot{m})\right|_{E X P}=1.54 \mathrm{~W}$ \\
\hline
\end{tabular}


Table 6: continued.

\begin{tabular}{|c|}
\hline$\left.g \Delta H_{\text {tot }}\left(\dot{m}+\dot{m}_{L}\right)\right|_{F L U E N T}=2.60 \mathrm{~W}$ \\
\hline$\left.g \Delta H_{\text {stat }}\left(\dot{m}+\dot{m}_{L}\right)\right|_{F L U E N T}=1.48 \mathrm{~W}$ \\
\hline$\left.\dot{W}_{I}\right|_{F L U E N T}=2.7 \mathrm{~W}$ \\
$\eta_{F L U}=\frac{1.48}{2.7}=54.8 \%$ \\
\hline$\eta_{H Y, F L U}=\frac{2.60}{2.70}=96.3 \%$ \\
$\eta_{\text {EXP }}=\frac{1.54}{5.73}=27 \%$ \\
$\eta_{\text {blower }}=\frac{1.54}{3.95}=39 \%$ \\
\hline
\end{tabular}

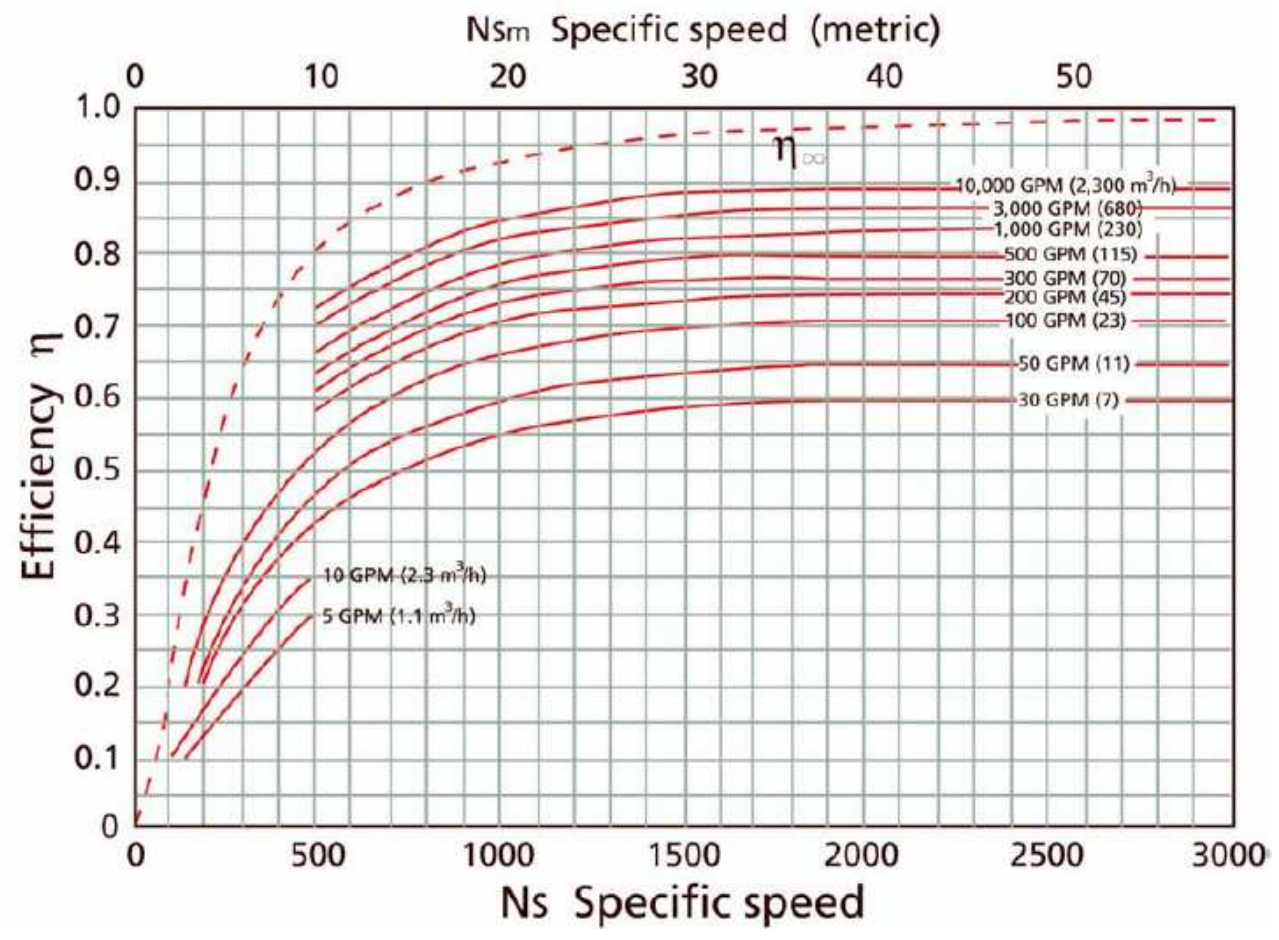

Figure 38: Efficiency values for pump with different specific speeds (Karassik et al. 2000, reprinted with permission of McGraw-Hill). 
The present hydraulic efficiency predicted by FLUENT is $96.3 \%$ and it points to a well designed blower. This high value can also be attributed to the fact that while calculating $\Delta H_{\text {tot }}$ FLUENT assumes no leakage flow i.e. a volumetric efficiency of $100 \%$, this may lead to an over-prediction of the term $\left(g \Delta H_{t o t}\left(\dot{m}+\dot{m}_{L}\right)\right)_{F L U E N T}$ used for efficiency calculations. Assuming certain leakage percentage, the numerator in Equation 36 is reduced and it affects a few percentage points on $\eta_{H Y, F L U}$.

Nonetheless, the overall blower design is better than what is shown in Figure 38 but the size needs to be changed to obtain the static pressure rise of $1000 \mathrm{~Pa}$. This is given next. Exact measurement of shaft power $\left(\dot{W}_{S}\right)$ using rotary transducers, to verify the efficiency values, can be part of any future work. This should consolidate the claim that the blower design is good.

\section{Resizing of Blower Using Enhanced-wall Treatment}

Blower simulations were redone for different sizes using the enhanced wall treatment approach. Since the overall blower efficiency is high, the simulation was done only at the design flow rate of $100 \mathrm{~L} / \mathrm{min}$. Table 7 shows the results.

Table 7: Static pressure values for different sized blowers at design flow rate.

\begin{tabular}{|c|c|c|}
\hline Blower Size & $\Delta P_{\text {stat }}(\mathrm{Pa})$ & $\dot{W}_{I}(\mathrm{~W})$ \\
\hline 65xz_26y & 884.0 & 2.9 \\
\hline 67xz_26y & 923.0 & 2.8 \\
\hline 68xz_26y & 966.4 & 3.0 \\
\hline 70xz_28y & 1043.0 & 3.4 \\
\hline
\end{tabular}


Since the results are under-predicted using the enhanced wall treatment approach, blower size $68 x z$ 26y seems the most appropriate. However, to allow for a small margin of safety, it was decided to use the70xz_28y. The blower will produce more than $1000 \mathrm{~Pa}$ when running at $20000 \mathrm{rpm}$. The rpm can be lowered to obtain the required pressure rise. 


\section{EXPERIMENTAL VALIDATION FOR 70XZ_28Y@20K BLOWER}

The test rig was kept the same as for the previously tested blower. After machining, the blower70xz_28y had an average (three readings) shroud diameter $\left(D_{2}\right)$ of1.888", and an average impeller inlet diameter $\left(d_{\text {in }}\right)$ of 1.200". A single front plate was used this time: Aluminum Plate \# 2 with Bigger Clearances $(A l-2: B C)$. Since the impeller diameter is bigger, the overall assembly had tighter clearances.

- $\delta_{\text {inlet }}=0.006^{\prime \prime}$ same as earlier case

- $\delta_{\text {shroud }}=0.013$ "for $A l-2: B C$ compared to 0.020 "for $A l-1: S C$

\section{Results}

Experimental results are presented in Figure 39 and experimental electrical power consumption in Figure 40. The experimental efficiency $\left(\eta_{E X P}\right)$ value is shown after that (Figure 41). The definition uses only the static head and ignores the velocity head for efficiency calculations. It is also based upon the electrical power provided to the motor and as such is the product of the blower and motor efficiency (Equation 33). It is a more realistic estimate of performance. 


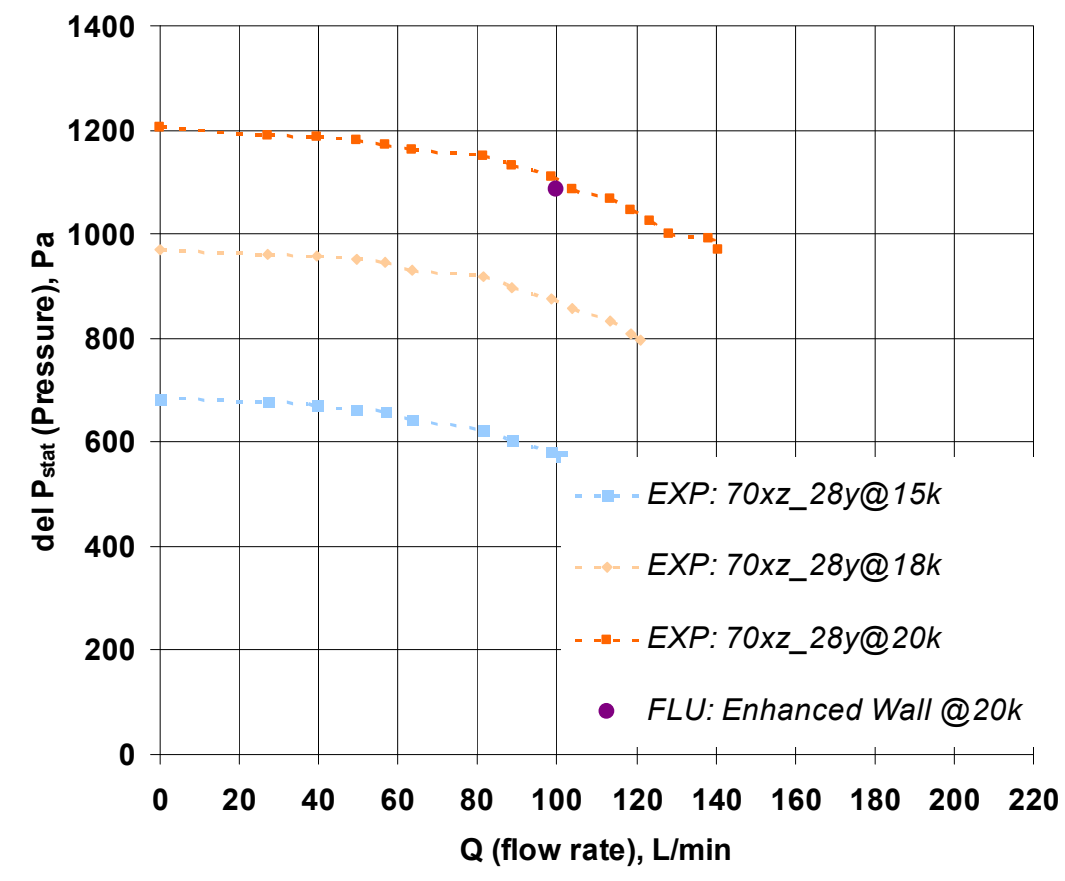

Figure 39: Experimental performance curve for 70xz_28y blower with Al-2: BC plate.

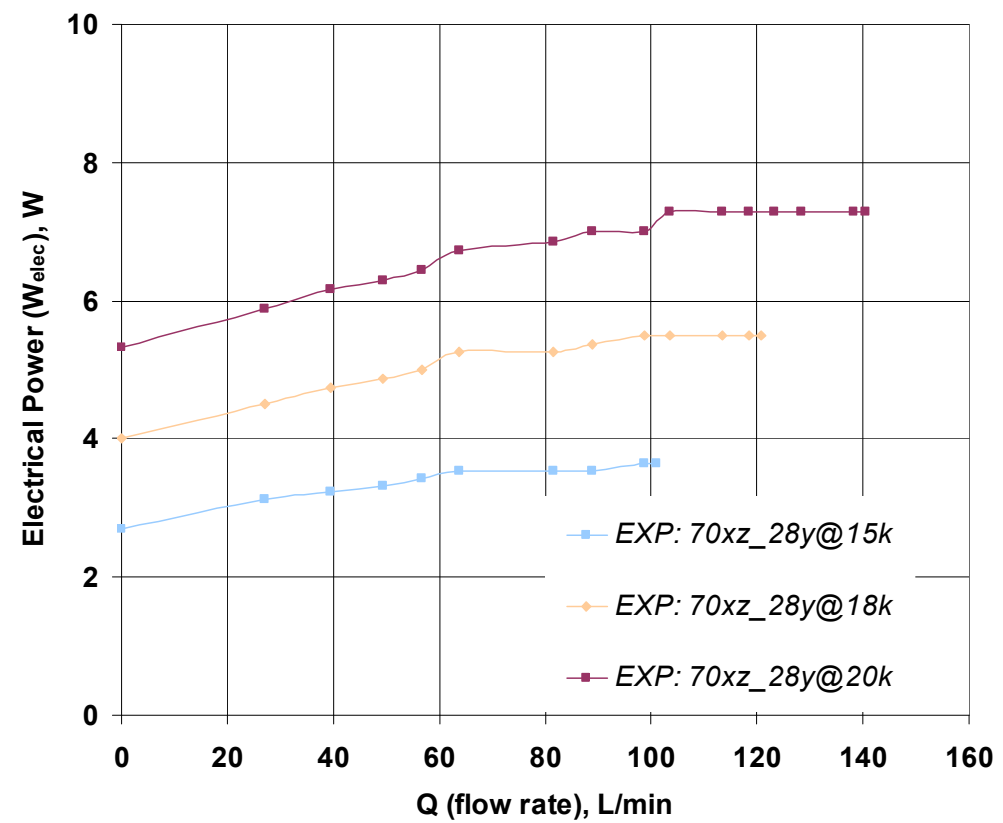

Figure 40: Experimental electrical power consumption curves for 70xz_28y blower with Al-2: BC front plate. 


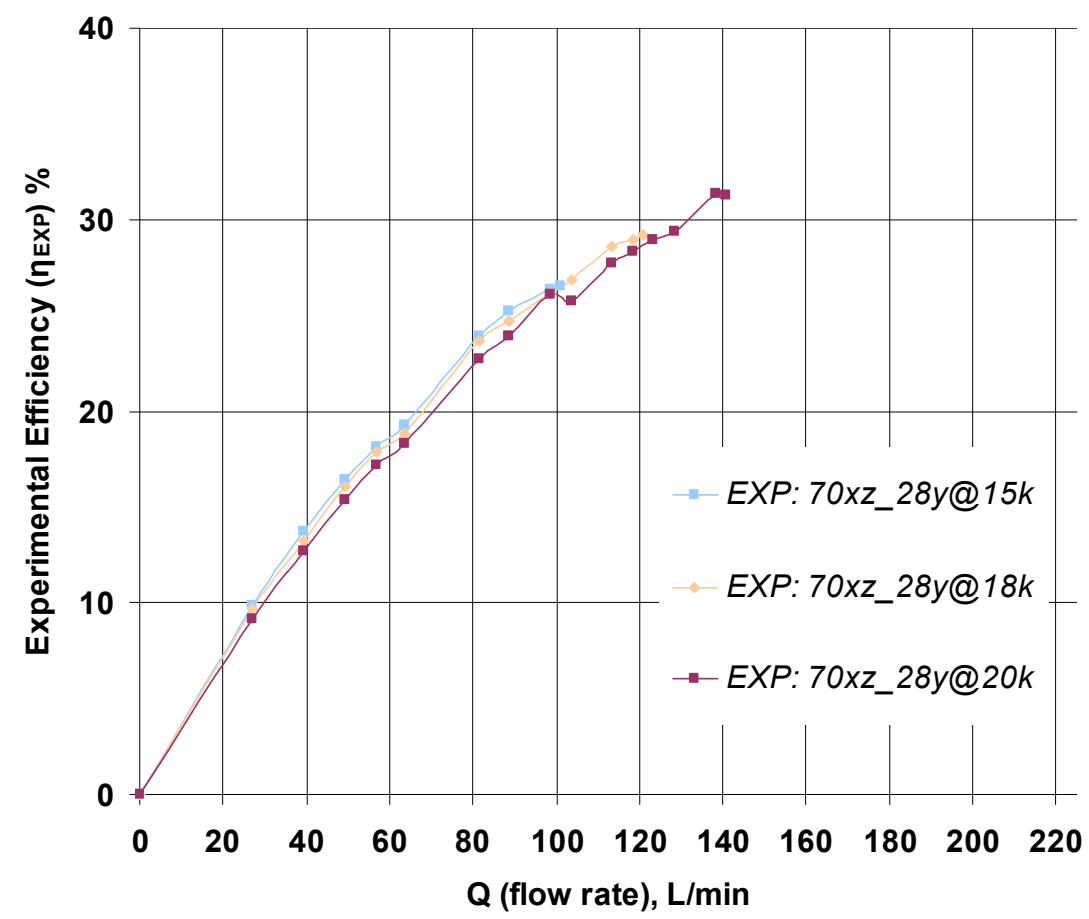

Figure 41: Experimental efficiency $\left(\eta_{E X P}\right)$ curves for 70xz_28y blower with Al-2: BC front plate.

\section{Conservative Efficiency Estimates for 70xz_28y@20k Blower}

An estimate of the two different blower efficiencies, namely, $\eta_{H Y, F L U}$ and $\eta_{F L U}$ is given below (Table 8). The blower specific speed at the design flow rate is $N_{s,(U S)}=932$ (Equation 39) gives the efficiency values:

$$
N_{s,(U S)}=\frac{N(\mathrm{rpm}) \sqrt{Q\left(\mathrm{GPM}_{\mathrm{US}}\right)}}{\left[\Delta H_{t o t}(\mathrm{ft})\right]^{3 / 4}}=\frac{(20000 \mathrm{rpm}) \cdot\left(26.4 \mathrm{GPM}_{\mathrm{US}}\right)^{0.5}}{(528.3 \mathrm{ft})^{3 / 4}}=932 \frac{\mathrm{rpm}^{3 / 4 P \mathrm{GP}_{\mathrm{US}}}{ }^{0.5}}{\mathrm{ft}^{0.75}}[39]
$$


Table 8: Blower efficiency estimates at design flow rate .

\begin{tabular}{|c|}
\hline Blower Size 70xz_28y@20k \\
\hline$\dot{W}_{\text {elec }}=7.0 \mathrm{~W}$ \\
\hline$\eta_{\text {motor }}=69 \%$ (best, data sheet) \\
\hline$\left.\Delta P_{\text {stat }}\right|_{E X P}=1111 \mathrm{~Pa}$ \\
\hline$\dot{W}_{S}=4.83 \mathrm{~W}$ \\
\hline$\left.g \Delta H_{\text {stat }}(\dot{m})\right|_{E X P}=1.85 \mathrm{~W}$ \\
\hline$\left.g \Delta H_{\text {tot }}\left(\dot{m}+\dot{m}_{L}\right)\right|_{F L U E N T}=3.23 \mathrm{~W}$ \\
\hline$\left.g \Delta H_{\text {stat }}\left(\dot{m}+\dot{m}_{L}\right)\right|_{F L U E N T}=1.74 \mathrm{~W}$ \\
\hline$\left.\dot{W}_{I}\right|_{F L U E N T}=3.3 \mathrm{~W}$ \\
\hline$\eta_{F L U}=\frac{1.74}{3.3}=52.7 \%$ \\
\hline$\eta_{H Y, F L U}=\frac{3.23}{3.3}=97.8 \%$ \\
$\eta_{E X P}=\frac{1.85}{7.0}=26.5 \%$ \\
\hline blower \\
$=\frac{1.85}{4.83}=38.3 \%$ \\
\hline
\end{tabular}

Value of $\eta_{H Y, F L U}$ is high. Further, while calculating $\Delta H_{\text {tot }}$ FLUENT assumes no leakage flow i.e. a volumetric efficiency of $100 \%$, this may lead to an over-prediction of the term $\left(\left.g \Delta H_{t o t}\left(\dot{m}+\dot{m}_{L}\right)\right|_{F L U E N T}\right.$ used for efficiency calculations. Further, at $20000 \mathrm{rpm}$ the value of $\eta_{H Y, F L U}$ is higher implying less separation and viscous losses. 


\section{Observations and Conclusions}

Important observations from this section are given below:

- Blower size 70xz_28y running at $20000 \mathrm{rpm}$ produces a static pressure rise of $1110 \mathrm{~Pa}$ experimentally, with the clearances as given by Al-2: BC front plate

- Blower efficiency is more than $38 \%$. The exact value can only be determined by making shaft torque measurements and total head rise measurements across the blower.

- FLUENT model with enhanced wall treatment shows blower 70xz_28y rotating at $20000 \mathrm{rpm}$ producing about 10\% more head than the design requirements of 1000 $\mathrm{Pa}$. This shows that a lower rpm value can be used; this is tested for the final prototype

- Controlling tip clearances is important, but a clearance at the blower inlet of $\delta_{\text {inlet }}=0.006$ " and on the shroud of $\delta_{\text {shroud }}=0.013^{\prime \prime}$ work fine. For the final prototype, $\delta_{\text {inlet }}$ is kept 0.009 "and $\delta_{\text {shroud }}$ at $0.012 "$. This had to be done as the final prototype was made out of Rapid Prototype (RP) material (plastic polymer) and controlling tolerances with plastic is difficult.

- Maximum liquid pump efficiency values, as given in Figure 38, can not be directly applicable to the present case but can only provide a benchmark

- Using a higher efficiency motor, a custom made brushless D.C motor, can help bring down the electrical power $\left(\dot{W}_{\text {elec }}\right)$ consumption.

- From the CFD point of view, enhanced wall function, with finer grid resolution, gives better results as compared to non-equilibrium wall function approach.

- Still higher static pressure rise can be achieved by converting the large dynamic head available at the blower exit. This can be done using a vaned stator or volute diffuser. Simulations and experiments were carried out using one such vaned diffuser, but no significant gain was observed. 


\section{FINAL BLOWER PROTOTYPE: DESIGN AND TESTING}

\section{Prototype Design Setup: Blower 70xz_28y with $\delta_{\text {inlet }}=0.009$ " and $\delta_{\text {shroud }}=0.012 "$}

For the final prototype the entire blower assembly was rapid prototyped. This was done keeping in mind the weight issues. Figure 42 shows the assembly. The overall diameter of the top diffuser plate is $3.5 "$. Engineering drawings for the blower are given in Appendix C.

\section{Final Prototype Assembly}

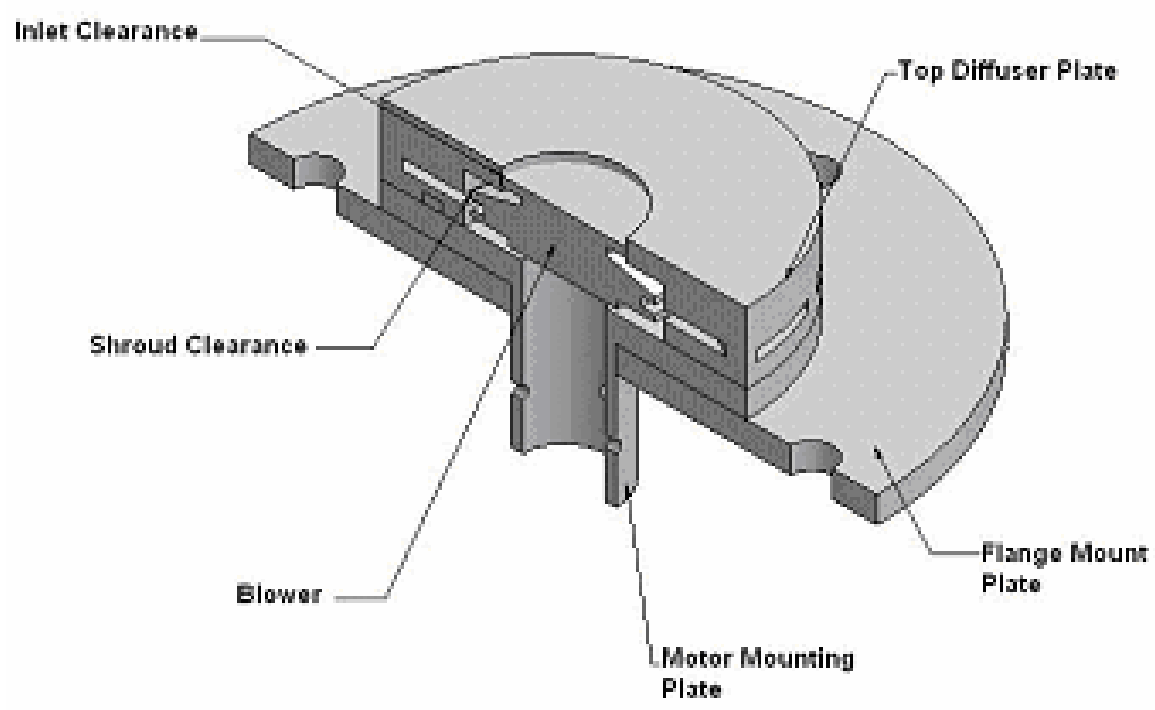

Figure 42: Final blower prototype test setup.

The metallic spacers have been removed; instead the diffuser plate has vanes. These vanes were designed to serve both as a support structure and for pressure recovery. Despite repeated testing and design modification, no pressure gain was observed. Nonetheless, these were retained to act as spacers between the top and the bottom halves of the diffuser plate, and also to point towards a future possibility. 
The tip clearances had to be controlled precisely. As the final prototype was fabricated out of a plastic-type polymer, it was difficult to accurately control the dimensions. Nonetheless, the tolerances were kept as tight as possible. Following values were used:

- Impeller diameter average $\left(D_{2}\right)$ of $1.888^{\prime \prime}$

- Average impeller inlet diameter $\left(d_{\text {in }}\right)$ of $1.200 "$

- $\quad$ Front plate inlet diameter $\left(d_{\text {in, plate }}\right)=1.218^{\prime \prime}$

- $\quad$ Plate shroud diameter $\left(d_{2, \text { plate }}\right)=1.912^{\prime \prime}$

The clearances one gets from these are:

- $\delta_{\text {inlet }}=0.009^{\prime \prime}$

- $\delta_{\text {shroud }}=0.012^{\prime \prime}$

Even though the shroud inlet clearance is more than the previous test cases, it appears to have no effect on the overall blower performance. The results given below show this point.

\section{Results}

As expected, running the impeller at $20000 \mathrm{rpm}$ produces higher pressure rise than needed. At $18000 \mathrm{rpm}$ the pressure produced is $900 \mathrm{~Pa}$ and at $20000 \mathrm{rpm}$ the pressure rise is about $1150 \mathrm{~Pa}$. Since pressure goes as $\Omega^{2}$, to produce $1000 \mathrm{~Pa}$ the rpm should be around $19000 \mathrm{rpm}$. Data tables are shown in Appendix D. Reducing the rpm also reduces the electrical power consumption from $7 \mathrm{~W}$ to $6.2 \mathrm{~W}$. At $19200 \mathrm{rpm}$ FLUENT with enhanced wall treatment gives pressure rise value of $\Delta P_{\text {stat }}=955.3 \mathrm{~Pa}$. The pressure rise value measured experimentally is $1021 \mathrm{~Pa}$. The percentage under-prediction is less than $6 \%$. This is good and it establishes faith in the CFD model being used. Figure 43 shows the experimental performance curves at different rpm. Electrical power consumption, measured experimentally is given in Figure 44, and Figure 45 shows the experimental efficiency values at different rpms. Experimental efficiency value for original hand-held vacuum system is also given. It shows a significant improvement. 


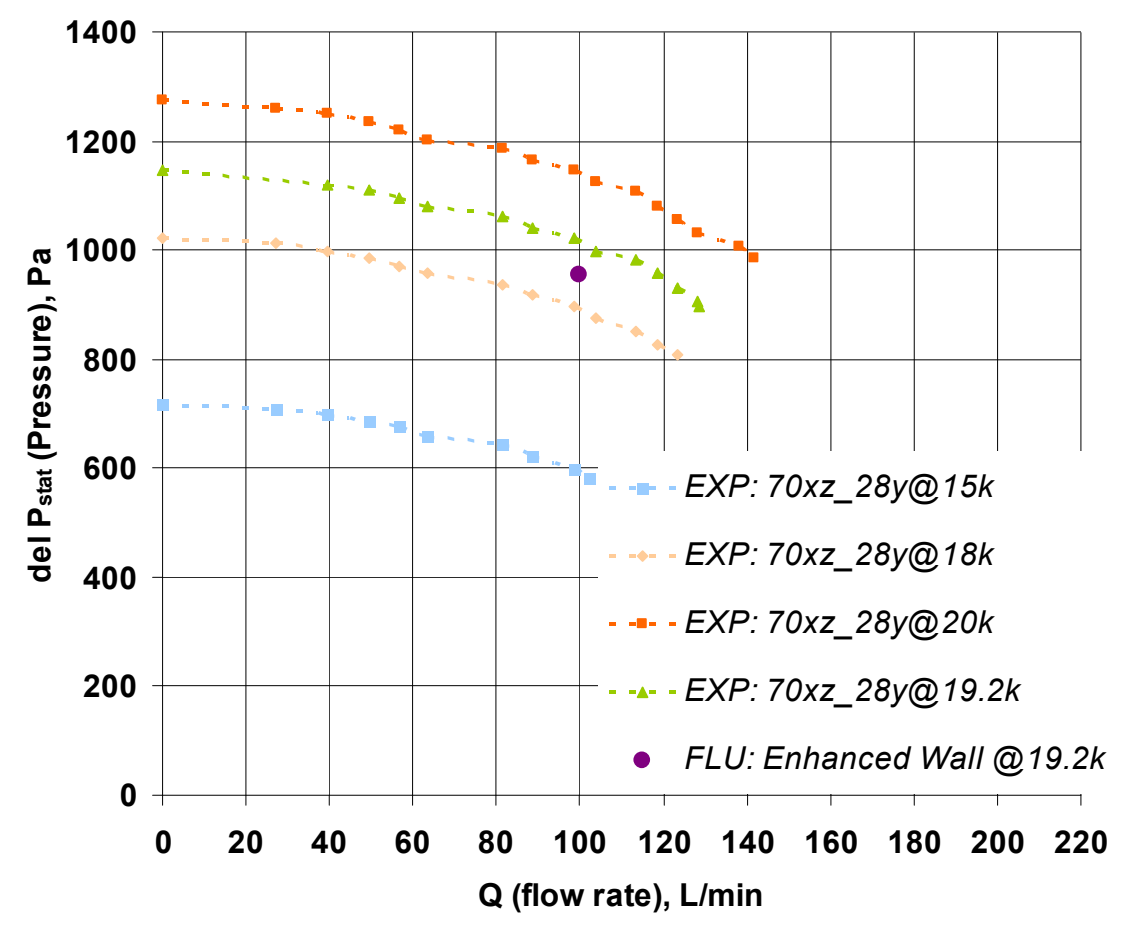

Figure 43: Experimental performance curves for final-prototype blower size 70xz_28y.

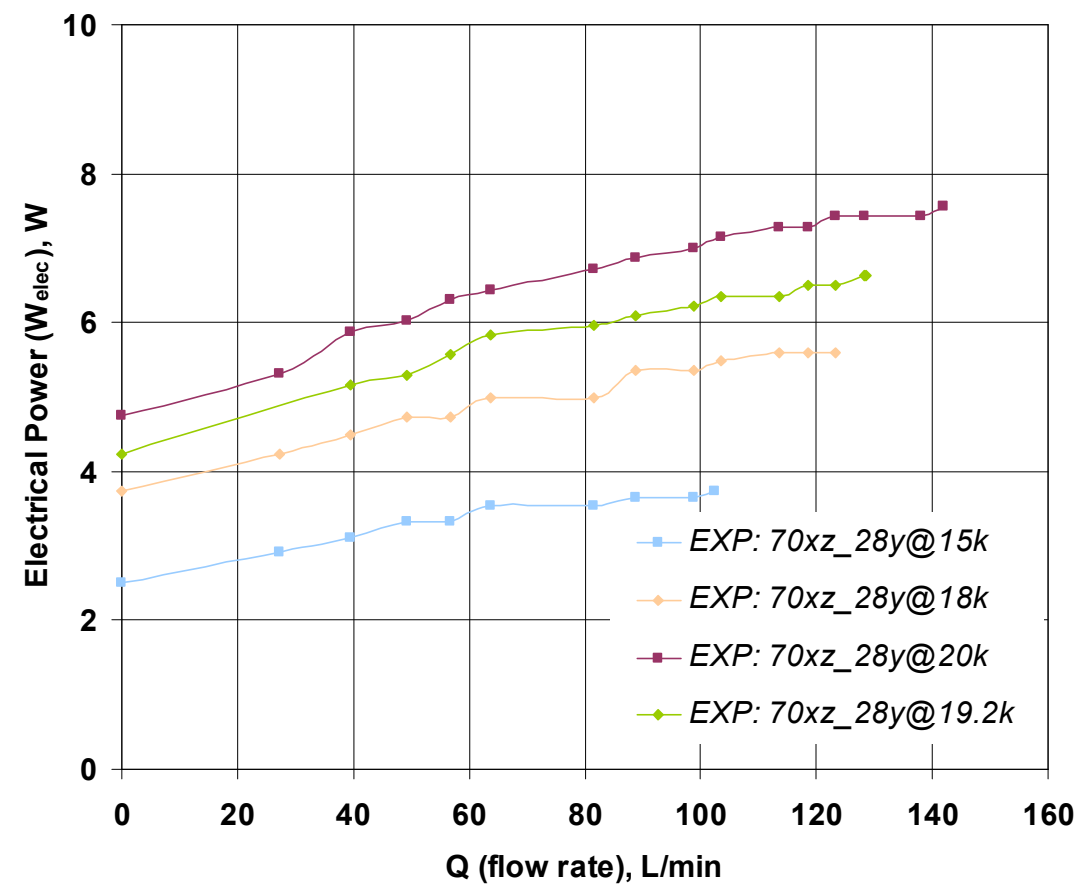

Figure 44: Experimental electrical power consumption curves for final-prototype. 


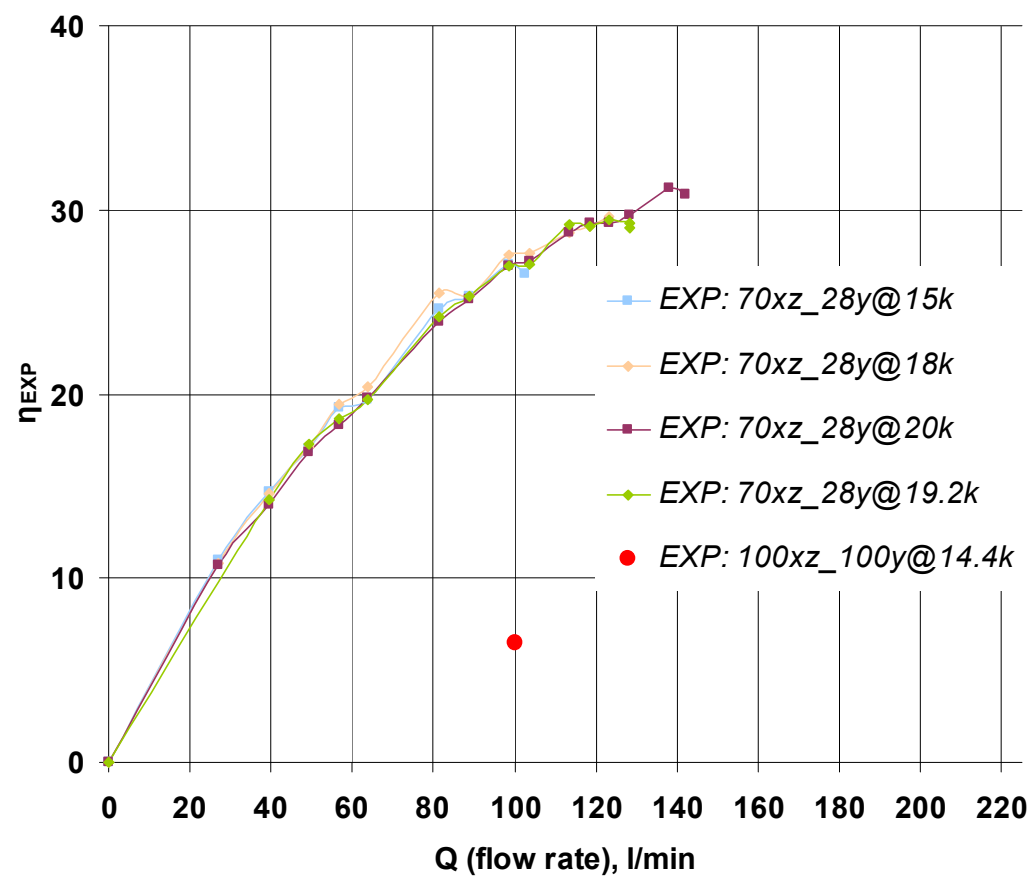

Figure 45: Experimental efficiency $\left(\eta_{E X P}\right)$ values for final-prototype $70 x z \_28 y$.

The specific speed of the blower running at $19200 \mathrm{rpm}$ is 965 (Equation 40). Results are given below and the efficiency estimates given in Table 9.

- $\Delta P_{\text {stat }}=1021 \mathrm{~Pa}$ from 70xz_28y@19.2k measured experimentally

- $\Delta P_{\text {stat }}($ FLUENT $)=955 \mathrm{~Pa}$ from enhance wall function approach.

- $\quad$ Percentage under-prediction $<6 \%$

$$
N_{s,(U S)}=\frac{N(\mathrm{rpm}) \sqrt{Q\left(\mathrm{GPM}_{\mathrm{US}}\right)}}{\left[\Delta H_{t o t}(\mathrm{ft})\right]^{3 / 4}}=\frac{(20000 \mathrm{rpm}) \cdot\left(26.4 \mathrm{GPM}_{\mathrm{US}}\right)^{0.5}}{(478.2 \mathrm{ft})^{3 / 4}}=965 \frac{{\mathrm{rpm} \cdot \mathrm{GPM}_{\mathrm{US}}}^{0.5}}{\mathrm{ft}^{0.75}}
$$


Table 9: Efficiency estimates for final prototype blower at design flow rate.

\begin{tabular}{|c|}
\hline Blower Size 70xz_28y@19.2k \\
\hline$\dot{W}_{\text {elec }}=6.23 \mathrm{~W}$ \\
\hline$\eta_{\text {motor }}=69 \%$ (best, data sheet) \\
\hline$\left.\Delta P_{\text {stat }}\right|_{\text {EXP }}=1021 \mathrm{~Pa}$ \\
\hline$\dot{W}_{S}=4.30 \mathrm{~W}$ \\
\hline$\left.g \Delta H_{\text {stat }}(\dot{m})\right|_{E X P}=1.7 \mathrm{~W}$ \\
\hline$\left.g \Delta H_{\text {tot }}\left(\dot{m}+\dot{m}_{L}\right)\right|_{F L U E N T}=2.92 \mathrm{~W}$ \\
\hline$\left.g \Delta H_{\text {stat }}\left(\dot{m}+\dot{m}_{L}\right)\right|_{F L U E N T}=1.60 \mathrm{~W}$ \\
\hline$\left.\dot{W}_{I}\right|_{F L U E N T}=3.0 \mathrm{~W}$ \\
\hline$\eta_{F L U}=\frac{1.60}{3.0}=53.3 \%$ \\
\hline$\eta_{H Y, F L U}=\frac{2.92}{3.0}=97.3 \%$ \\
$\eta_{E X P P}=\frac{1.7}{6.23}=27.3 \%$ \\
\hline blower
\end{tabular}

From Figure 45, the efficiency improvement observed over the original 100xz_100y system is more than $2 \mathrm{X}$. This can be attributed to the better customization of blower size to meet the design condition, and a higher efficiency motor that was used. The blower certainly outperforms the efficiency values given in Figure 38. 


\section{SUMMARY AND CONCLUSIONS}

Design, development, and testing of blowers for future aerosol applications were carried out. Designing a blower to meet $100 \mathrm{~L} / \mathrm{min}$ of flow rate and a static pressure rise of 1000 Pa was challenging. Lack of literature for such portable applications and dearth of commercially available blowers to meet such low flow rate and pressure rise values made the research necessary. A top-down design approach was adapted and it included deciding the design objectives, selecting a blower type, designing a blower and testing and validation. During the course of the study, the applicability of computational fluid dynamics (CFD) to turbomachine design was established. Incorporating CFD into the design process shortens the design cycle and adds a new dimension allowing the visualization of flow field within the impeller.

Major findings from the study are given below:

- Maximum overall efficiency considerations established the blower type to be a centrifugal impeller

- Optimum blower performance pointed towards a high rpm blower. Overall the trend supported faster, smaller blowers.

- At high rpm values the blower static head cannot be ignored. High velocity head adds to the overall efficiency. At the same time, the kinetic energy is not utilized since the blower exhausts to the atmosphere.

- The approach towards blower design, adopted in the literature, is the modification of an existing design rather than designing from base zero.

- Hand held vacuum systems, being battery operated, have blowers that are the product of good engineering design. One such blower was used as a base-design

- CFD package, FLUENT, allowed the non-traditional scaling to be performed. A blower $70 \%$ scaled in the $X$ and $Z$ directions and $28 \%$ scaled in $Y$ running at $19,200 \mathrm{rpm}$ was found to be the most appropriate 
- Blower size 70xz_28y running at 19,200 rpm produces a static pressure rise of $1021 \mathrm{~Pa}$

- It was found that the use of standard wall function approach (non-equilibrium wall functions) over predicted the static pressure rise.

- FLUENT results allowed the blade height to be incorporated into the traditional fan scaling equations. This lead to modified fan scaling laws. These relationships are only from the simulated data and need to be modified.

- Finer grid resolution with the enhance wall function approach gave acceptable agreement between simulations and experiments.

- Tip clearance, especially at the impeller inlet and shroud surfaces, significantly affect blower performance

- Hydraulic efficiency value $\left(\eta_{H Y, F L U}\right)$, based on the total head values given by FLUENT is $98.12 \%$ at $19200 \mathrm{rpm}$.

Important inferences that can be drawn are:

- Design approach based on modifying an initial base-design is effective in meeting the design requirements.

- CFD shortens the entire design process allowing the analysis of different scaling cases with relative ease.

- Maximum liquid pump efficiency values, as given in Figure 38, may not be directly applicable for the present case. In the absence of such charts for air blowers, Figure 38 acts as a good benchmark

- Efficiency values for smaller blowers tend to reduce due to scale effect. CFD overcomes this limitation by allowing non-traditional scaling.

- From the CFD point of view, enhanced wall function, with finer grid resolution, gives better results as compared to non-equilibrium wall function approach.

- Controlling tip clearances is important for better performance

- Still higher static pressure rise can be achieved by converting the large dynamic head available at the blower exit. This can be done using a vaned stator or volute diffuser. This won't affect the overall efficiency value. 


\section{RECOMMENDATIONS FOR FUTURE WORK}

The work on this project is by no means complete. Designing and performance improvement of air blowers for future aerosol samplers can go a long way. One probable area is to establish overall efficiency versus specific speed graphs at different flow rates.

Another significant area is experimentally determining the overall blower efficiency values. This can be done by measuring the shaft torque values using rotary transducers or other such devices. For calculating efficiency values in this thesis, total head values predicted by FLUENT were used. Even though, pressure predictions from FLUENT were within acceptable error range, there is no way to validate the total head rise values given by FLUENT. Experimental test-rig needs to be modified to be able to measure total head change across the impeller. This will allow $\eta_{\text {overall }}$ to be determined and compared with Figure 36 values. This will validate the performance improvement in the blower.

Clearance control on the impeller inlet and shroud affects performance significantly. For any future prototypes, these values need to be controlled precisely. A recommendation would be using aluminum front plate for better dimensional control. Final prototype was made using plastic type resin and maintaining the precise tolerances on these was difficult due to low machinability, moisture absorption, and temperature variations in the surroundings.

Another area that can produce great performance level improvements is having a better efficiency motor. A custom made D.C. brushless motor with high motor efficiency values can go a long way in bringing down the D.C. power consumption. Looking into this domain can be a possible next step. 


\section{REFERENCES}

Anderson, H. H. (1980). Prediction of Head, Quantity, and Efficiency in Pumps - The Area Ratio Principle. Performance Prediction of Centrifugal Pumps and Compressors, ASME. 1980, $201-211$

Black, R. (2002). Development of the Wet Walled Cyclone Collector for the Joint Biological Point Detection System, Presentation to the 2002 Scientific Conference on Obscuration and Aerosol Research, Edgewood, MD.

Balje, O. E. (1962). A Study on Design Criteria and Matching of Turbomachinery, ASME Journal of Engineering for Power. January, 83-102, 103-114.

Bleier, Frank, B. (1997). Fan Handbook: Selection, Application, and Design, McGraw Hill, New York.

Cartwright, W. G. (1977). Specific Sped as a Measure of Design Point Efficiency and Optimum Geometry for a Class of Compressible Flow Turbomachines, Scaling for Performance Prediction in Rotodynamic Machines, Institution of Mechanical Engineers, New York, 139-145

Engeda, A. (1995). Correlation and Prediction of Efficiency of Centrifugal Pumps due to Tip Clearance Effects. Proc. of Institute of Mechanical Engineers. Vol. 20. 111-114

ERCOFTAC Best Practice Guidelines for Industrial Computational Fluid Dynamics version 1 (2000). European Research Community on Flow, Turbulence, and Combustion

European Commission Report (2001) Study on Improving the Energy Efficiency of Pumps, February 2001 AEAT-6559/ v 5.1

FLOW-DYNE Engineering, Inc., Fort Worth, URL: http:/www.flow-dyne.com. Accessed August, 2004.

FLUENT 6.1.22. (2004). FLUENT Inc, Lebanon, NH

FLUENT User's Guide Version 6.1.22. (2004). FLUENT, Inc, Lebanon, NH.

GAMBIT 2.1 User's Guide (2004). FLUENT, Inc, Lebanon, NH

General Dynamics, Armament and Technical Products (2004). Joint Biological Point Detection System (JBPDS), URL: http://www.gdatp.com/Products/PDFs/JBPDS.pdf 
Graf, E. (1993). Analysis of Centrifugal Impeller BEP and Recirculating Flows: Comparison of Quasi-3D and Navier-Stokes Solutions. Pumping Machinery - 1993. FED - Vol. 154, ASME, 1993, 235 - 245.

Gülish, J. F., Favre, J. N., and Denus, K. (1997). An Assessment of Pump Impeller Perfor-mance Predictions by 3D-Navier-Stokes Calculations. 1997 ASME Fluids Enginee-ring Division Summer Meeting. Paper FEDSM97 - 3341, June 1997

Haglund, John S. and McFarland, Andrew R. (2004). A Circumferential Slot Virtual Impactor, Aerosol Sci. Technology. 38:664 - 674.

Halstead, C. (2004). Fan Selection -A Practical Guide. Intern. Conf. on Fans, The Fluid Machinery Group of the Institution of Mechanical Engineers, London 283 - 296

Han, Hai, Yokoyama, Yoichi, Konno, Brad M. (2002). Recent Developments and Applications in CFD for Turbomachinery, 4th International Conference on Pumps and Fans ( $\left.4^{\text {th }} \mathrm{ICPF}\right)$, Tsinghua University, Beijing

Hydraulics Institute, URL: http://www.pumps.org. Accessed January, 2005.

Inventor Professional 8 (2002), Autodesk Inc, San Rafael, CA

Isaguirre, Refugio R. (2004). An Experimental Study of Linear Slot Circumferential Virtual Impactors for use in Continuous Bioaerosol Concentration, M.S. Thesis, Texas A\&M University, College Station

Japikse, David, Marscher, William D., Furst, Raymond B. (1997). Centrifugal Pump Design and Performance, Concepts ETI, Inc.

Karassik, Igor J., Messina, Joseph P., Cooper, Paul, Heald Charles C. (2000). Pump Handbook, McGraw Hill Professional, New York, $3^{\text {rd }}$ ed.

Katsanis, T. (1991). Quasi-Three-Dimensional Full Analysis in Turbomachines: A Tool for Blade Design. Numerical Simulations in Turbomachinery, FEF - Vol. 120, ASME, 1991, $57-64$

Koh, C. P., Chan, W. K, Ng, B. T. H, Li, H. D. (1999). Design and Investigation of Flow Field in a Centrifugal Blood Pump. ASME, Bioengineering Division. BED, Vol. 42, $1999,167-168$. 
Lakshminarayana, B. (1991). An Assessment of Computational Fluid Dynamic Techniques in the Analysis and Design of Turbomachinery - The 1990 Freeman Scholar Lecture. J. of Fluids Engineering, Vol. 113: 315 - 352

Launder, B. E., and Spalding. D. B. (1972) Lectures in Mathematical Models of Turbulence. Academic Press, London.

Lelkes, A. (2004). Novel Concepts for Improved Brushless Fan Motors. Intern. Conf. on Fans, The Fluid Machinery Group of the Institution of Mechanical Engineers, London $73-84$

Mann, D. (2004). Fan Technology - Evolutionary Potential and Evolutionary Limits. Intern. Conf. on Fans, The Fluid Machinery Group of the Institution of Mechanical Engineers, London $225-235$

Moncla, B. (2004). Personal communication, Aerosol Technology Laboratory, Texas A\&M University, College Station.

Pope, Stephen B. (2001). Turbulent Flows, Cambridge University Press, Cambridge.

Sabersky, R., and Acosta, A. J. (1966). Fluid Flow, Macmillan.

Tallgren, J. A., Sarin, D. A., and Sheard, A. G. (2004). Utilization of CFD in Development of Centrifugal Fan Aerodynamics. Intern. Conf. on Fans, The Fluid Machinery Group of the Institution of Mechanical Engineers, London $99-118$

Thakur, Siddharth, Lin Wanlai, and Jeffrey Wright. (2002). Prediction of Flow in Centrifugal Blower Using Quasi-Steady Rotor-Stator Models. J. of Eng. Mechanics, October, 1039-1049

Tennekes, H. and Lumley, J. L. (1997). A First Course in Turbulence, MIT Press Design Department, Cambridge, Massachusetts.

Turton, R. K. (1984). Principles of Turbomachinery, E. \& F. N. Spon, New York.

Tuzson, John. (2000). Centrifugal Pump Design, A Wiley-Interscience publication, John Wiley \& Sons, Inc.

Wood, G. M., Welna, H. and Lamers, R. P. (1965). Tip Clearance Effects in Centrifugal Pumps. ASME Tran., 85

Yakhot, V. and Orszag, S. A. (1986). Renormalization Group Analysis of Turbulence: I. Basic Theory. Journal of Scientific Computing, 1(1):1-51 


\section{APPENDIX A}

\section{VENTURI CALIBRATION CHART}

The calibration chart provided by the venturi company (FLOW-DYNE Engineering, Inc) is given in Figure A-1. A sample calculation follows below.

\section{Data Reduction}

In using venturi for measuring flow rates, it is necessary to take 3 measurements pertinent to flow condition. These are: 1) the inlet static pressure; 2) the inlet total temperature of the flow media; and 3) the static pressure differential between the throat and the inlet. To obtain the $100 \mathrm{~L} / \mathrm{min}$ volume flow rate the following conditions are chosen:

Table A-1: Values chosen for mass flow calculation.

\begin{tabular}{|l|l|}
\hline Throat diameter $\left(D_{2}\right)$ & $0.25^{\prime \prime}$ \\
\hline Inlet Static Pressure $\left(P_{1}\right)$ & $14.7 \mathrm{psia}$ \\
\hline Metering differential $(\Delta P)$ & $6^{\prime \prime} \mathrm{WC}=0.217 \mathrm{psia}$ \\
\hline Inlet Temperature $\left(T_{O 1}\right)$ & $74^{\circ} \mathrm{F}=534^{\circ} R$ \\
\hline
\end{tabular}

$\frac{\Delta P}{P_{1}}=\frac{0.217}{14.7}=0.01475$. Project a horizontal line thru $\frac{\Delta P}{P_{1}}=0.01475$ on the air-curve (Figure A-1).At the point where this line intersects the curve $D_{2}=0.25 "$, drop a vertical line to the $\frac{W \sqrt{T_{O 1}}}{P_{1}}$ scale and $\operatorname{read} \frac{W \sqrt{T_{O 1}}}{P_{1}} \approx 0.4$. Then solve for flow rate: $\frac{W \sqrt{T_{O 1}}}{P_{1}} \approx 0.4 ; W=0.4 \frac{P_{1}}{\sqrt{T_{O 1}}}=0.4 \frac{14.7}{\sqrt{534}}=0.25445 \mathrm{lbm} / \mathrm{min}=0.11542 \mathrm{~kg} / \mathrm{min}$. This is converted to volume flow rate using an air density value of $\rho_{\text {air }}=1.17 \mathrm{~kg} / \mathrm{m}^{3}$ to get a flow rate of $98.7 \mathrm{~L} / \mathrm{min}$. Manometer range is selected by back calculating the pressure differential for the maximum flow rate expected. 
YENTUH FLOW RANGE FOR AIR

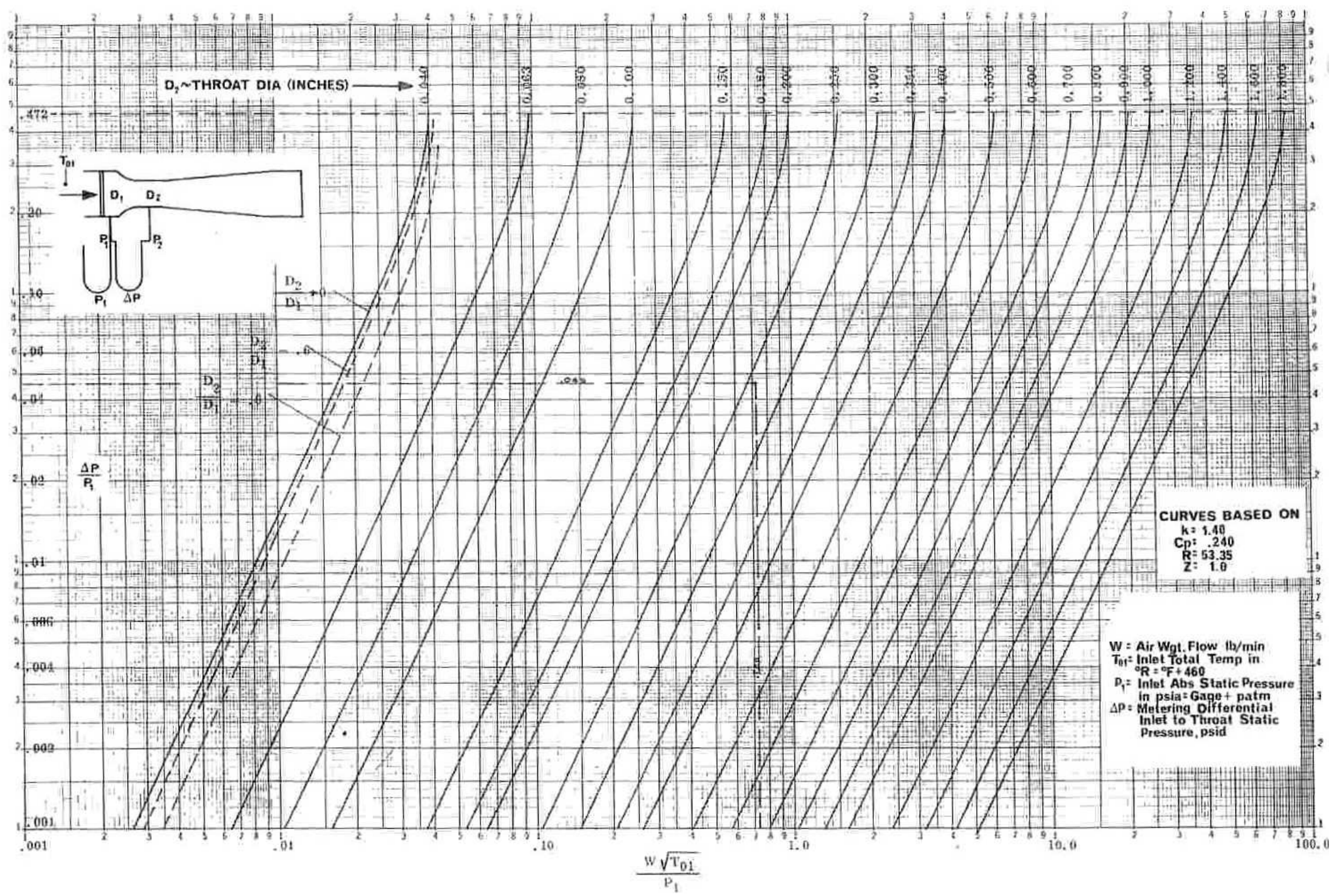

Figure 46: Venturi calibration chart. 


\section{APPENDIX B}

\section{MOTOR DATA SHEETS}

Brushless D.C. motors available from MicroMo Electronics (http://www.micromo.com) used as blower drive. Data charts for the motor used, $2036024 B$ is given in Figure B-1. The maximum motor efficiency value $\left(\eta_{\text {motor }}\right)$ used in the thesis is referenced from these data sheets.

Data sheets for the other motor, $1628024 B$ mentioned in the thesis can be obtained directly from the homepage of MicroMo Electronics. 
EN FAULHABER

\section{Brushless DC-Servomotors}

Electronic Commutation

\section{$0.74 \mathrm{oz}$-in}

For combination with Gearheads: 20/1

Encoders: IE2-512, 5500, 5400, HEDL 5540 Drive Electronics: BLD 3502, BLD 5604, BLD 5603,

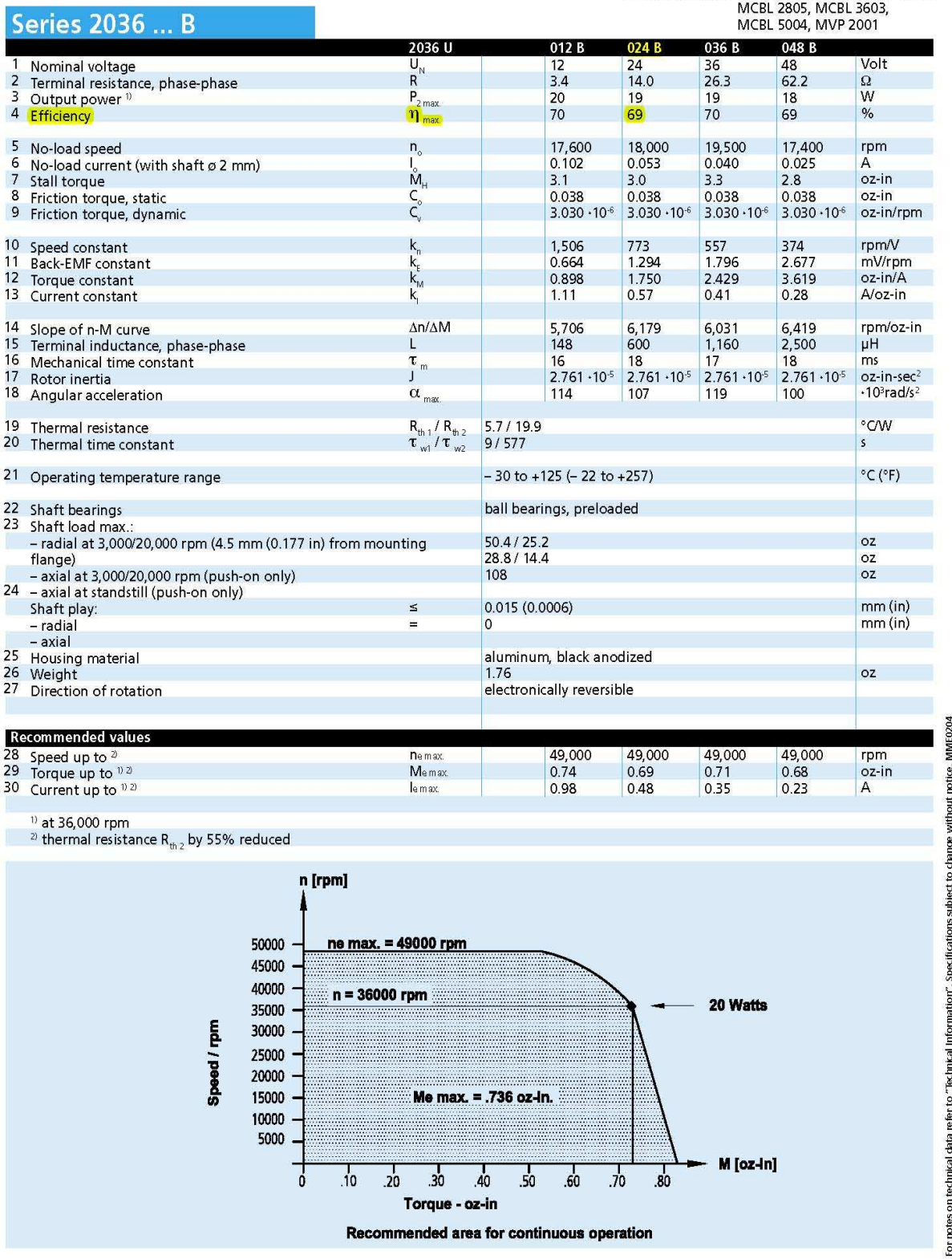

Microlo Electronics, Inc · 14881 Evergreen Avenue · Cleanwater · FL 33762-3008 · Toll-Free: (800) 807-9166 · Fax: (727) 573-5918 · info@micromo.com · wnmw micromo.com

Figure B-1: $2036024 B$ motor data sheet. 


\section{APPENDIX C}

70xz_28y Final Blower Prototype Drawings

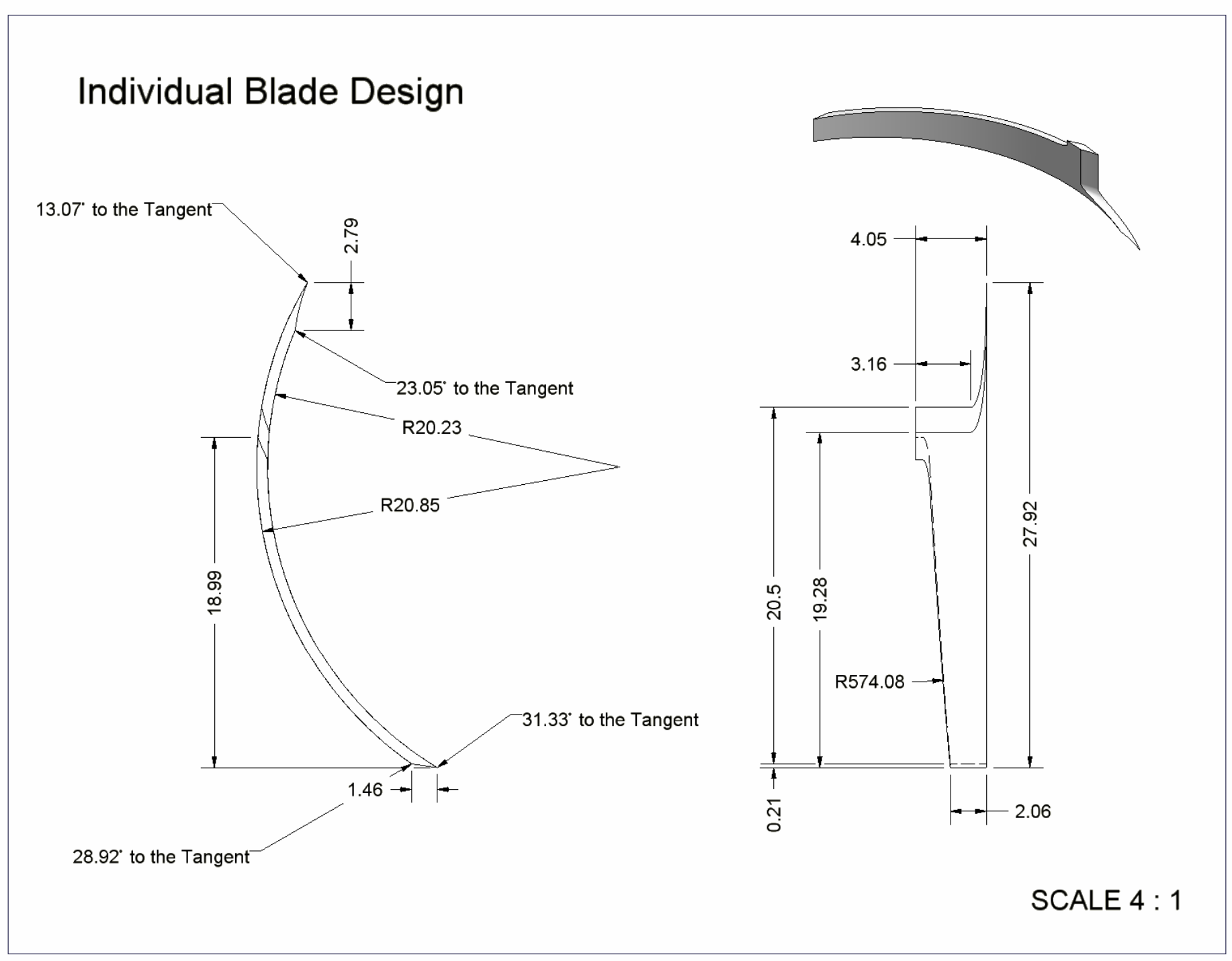

Figure C-1: Blade dimensions (mm) for 70xz_28y final prototype. 


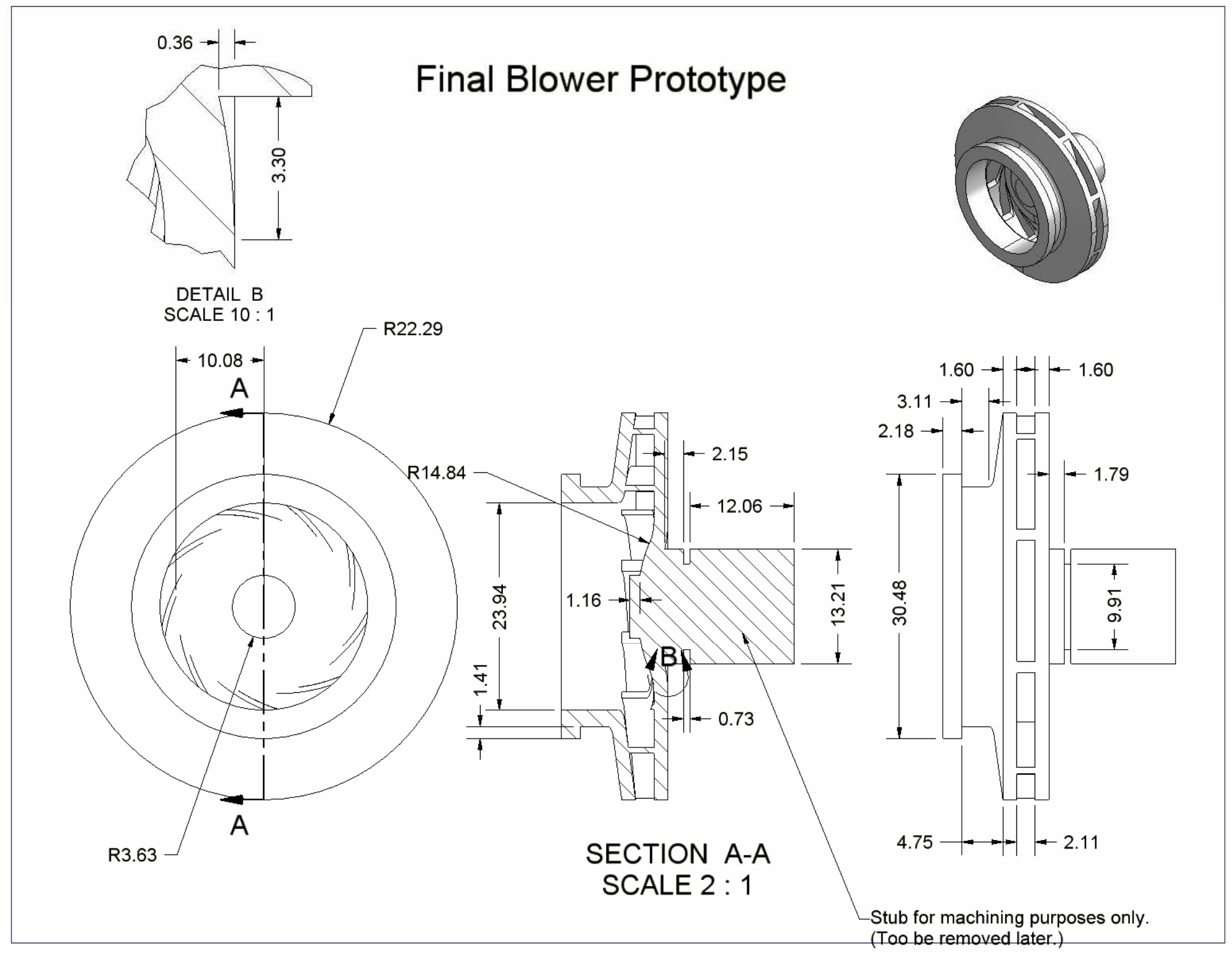

Figure C-2: 70xz_28y final prototype dimensions (mm). 


\section{APPENDIX D}

\section{SAMPLE DATA SHEETS}

Sample data sheet for the final prototype blower 70xz_28y running at 19,200 rpm is given below (Table D-1). The nomenclature used is given below:

- $T=534^{\circ} \mathrm{R}$

- $\quad P_{\text {atm }}=14.7 \mathrm{psi}$

- $\quad \Delta P=$ Pressure difference across venturi ("WC)

- $Y=\frac{\Delta P(\mathrm{psi})}{P_{a t m}(\mathrm{psi})} \mathrm{Y}$-axis value on venturi chart

- $\quad X=\mathrm{X}$-axis intercept on venturi chart

- $\quad \dot{m}(\mathrm{lbm} / \mathrm{min})=$ mass rate from flow chart

- $\rho_{\text {air }}=1.7 \mathrm{~kg} / \mathrm{m}^{3}$

- $Q(\mathrm{~L} / \mathrm{min})=$ flow rate calculated from $\dot{m}(\mathrm{lbm} / \mathrm{min})$

- $\quad P_{\text {stat }}(\mathrm{Pa})=$ suction pressure at blower inlet

- $\quad V(\mathrm{~V})=$ Voltage supplied to power supply

- $\quad I(\mathrm{Amp})=$ Current drawn by motor

- $\dot{W}_{\text {elec }}(\mathrm{W})=$ Electrical power given to motor $=V . I$

- $\quad \dot{W}_{\text {hydraulic }}(\mathrm{W})=$ Hydraulic power generated by blower $\left(=Q . P_{\text {stat }}\right)$

- $\eta_{E X P}$ 
Table D-1: Sample data sheet for final prototype blower at design rpm.

\begin{tabular}{|c|c|c|c|c|c|c|c|c|c|c|}
\hline \multicolumn{10}{|c|}{ Blower 70xz_28y @ 19200rpm } \\
\hline$\Delta P$ & $Y$ & $X$ & $\dot{m}$ & $Q$ & $P_{\text {stat }}(\mathrm{Pa})$ & $V$ & $I$ & $\dot{W}_{\text {elec }}(\mathrm{W})$ & $\dot{W}_{\text {hydraulic }}(\mathrm{W})$ & $\eta_{\text {EXP }}$ \\
\hline 0 & 0.00000 & 0 & 0.00 & 0 & 1145.8 & 26.5 & 0.16 & 4.24 & 0 & 0.00 \\
\hline 0.5 & 0.00123 & 0.11 & 0.07 & - & - & - & - & - & - & - \\
\hline 1 & 0.00246 & 0.16 & 0.11 & 39.5 & 1120.9 & 26.5 & 0.195 & 5.1675 & 0.74 & 14.27 \\
\hline 1.5 & 0.00369 & 0.2 & 0.13 & 49.3 & 1110.9 & 26.5 & 0.2 & 5.3 & 0.91 & 17.24 \\
\hline 2 & 0.00492 & 0.23 & 0.15 & 56.7 & 1096.0 & 26.5 & 0.21 & 5.565 & 1.04 & 18.63 \\
\hline 3 & 0.00737 & 0.258 & 0.16 & 63.6 & 1081.0 & 26.5 & 0.22 & 5.83 & 1.15 & 19.67 \\
\hline 4 & 0.00983 & 0.33 & 0.21 & 81.4 & 1061.1 & 26.5 & 0.225 & 5.9625 & 1.44 & 24.15 \\
\hline 5 & 0.01229 & 0.36 & 0.23 & 88.8 & 1041.2 & 26.5 & 0.23 & 6.095 & 1.54 & 25.29 \\
\hline 6 & 0.01475 & 0.4 & 0.25 & 98.7 & 1021.3 & 26.5 & 0.235 & 6.2275 & 1.68 & 26.97 \\
\hline 7 & 0.01720 & 0.42 & 0.27 & 103.6 & 996.4 & 26.5 & 0.24 & 6.36 & 1.72 & 27.05 \\
\hline 8 & 0.01966 & 0.46 & 0.30 & 113.5 & 981.4 & 26.5 & 0.24 & 6.36 & 1.86 & 29.19 \\
\hline 9 & 0.02212 & 0.48 & 0.31 & 118.4 & 956.5 & 26.5 & 0.245 & 6.4925 & 1.89 & 29.08 \\
\hline 10 & 0.02458 & 0.5 & 0.32 & 123.3 & 931.6 & 26.5 & 0.245 & 6.4925 & 1.92 & 29.50 \\
\hline 11 & 0.02703 & 0.52 & 0.33 & 128.3 & 906.7 & 26.5 & 0.25 & 6.625 & 1.94 & 29.26 \\
\hline 11.04 & 0.02713 & 0.521 & 0.33 & 128.5 & 896.7 & 26.5 & 0.25 & 6.625 & 1.92 & 29.00 \\
\hline
\end{tabular}




\section{VITA}

Name:

Raman Chadha

Email:

raman.chadha@gmail.com

Address:

Department of Mechanical Engineering

Texas A\&M University

Mail Stop 3123

College Station, Texas 77843-3123

Education:

M.S. Mechanical Engineering (December 2005)

Texas A\&M University (http://www.tamu.edu)

College Station, Texas 77843-3123

B-En., Mechanical Engineering (May 2003)

IIT Kanpur (http://www.iitk.ac.in)

Kanpur, India

Work Experience: 06/04-12/05 Graduate Research Assistant

Aerosol Technology Laboratory

Mechanical Engineering Department

Texas A\&M University

College Station, Texas 77843-3123

08/03-05/04 Teaching Assistant

Thermodynamics Fluid Analysis and Design

(MEEN-421)

Mechanical Engineering Department

Texas A\&M University

College Station, Texas 77843-3123 